\title{
Resultados de existência para alguns problemas não lineares com valores na fronteira de equações diferenciais
}

\author{
Dionicio Pastor Dallos Santos \\ TESE APRESENTADA \\ $\mathrm{AO}$ \\ Instituto de Matemática e Estatística \\ DA \\ Universidade DE SÃo PAUlo \\ PARA \\ OBTENÇÃO DO TÍTULO \\ $\mathrm{DE}$ \\ DOUTOR EM CIÊNCIAS \\ Programa: Matemática \\ Orientador: Prof. Dr. Pierluigi Benevieri
}

Durante o desenvolvimento deste trabalho o autor recebeu auxílio financeiro da CAPES/CNPq

São Paulo, Maio de 2017 


\section{Resultados de existência para alguns problemas não lineares com valores na fronteira de equações diferenciais}

Esta tese trata-se da versão original do aluno

(Dionicio Pastor Dallos Santos). 


\title{
Resultados de existência para alguns problemas não lineares com valores na fronteira de equações diferenciais
}

\begin{abstract}
Esta versão da tese contém as correções e alterações sugeridas pela Comissão Julgadora durante a defesa da versão original do trabalho, realizada em 26/05/2017. Uma cópia da versão original está disponível no Instituto de Matemática e Estatística da Universidade de São Paulo.
\end{abstract}

Comissão Julgadora:

- Prof. Dr. Pierluigi Benevieri - IME-USP

- Prof. Dra. Jaqueline Godoy Mesquita - UnB

- Prof. Dra. Márcia Federson - ICMC-USP

- Prof. Dr. Antônio Luiz Pereira - IME-USP

- Prof. Dr. João Marcos Bezerra do Ó - UFPB 


\section{Agradecimentos}

Agradeço a toda minha família, em especial à minha mãe, María Soledad Santos, pelo carinho e amor que sempre me deu.

A meu orientador, Pierluigi Benevieri, pelo papel fundamental que cumpriu na minha formação como matemático.

Agradeço também à CAPES e CNPq/Brasil pelo apoio financiero. 


\section{Resumo}

DALLOS, D. P. Resultados de existência para alguns problemas não lineares com valores na fronteira de equações diferenciais. 2017. 87 f. Tese (Doutorado) - Instituto de Matemática e Estatística, Universidade de São Paulo, São Paulo, 2017.

O principal objetivo deste trabalho é estudar a existência de soluções para alguns problemas de valores de contorno de equações diferenciais ordinárias não lineares em dimensão finita e infinita. Todos os sistemas considerados nesta investigação são transformados em equações funcionais nas quais o objetivo é encontrar um ponto fixo de um oportuno operador definido em um espaço de funções (que depende do problema estudado). Para isso, faremos uso do grau de Leray-Schauder e de um conceito de grau topológico, devido a R. Nussbaum, para perturbações não compactas da identidade em espaços de Banach.

Os resultados novos apresentados neste trabalho estão contidos em 5 artigos, dos quais dois já foram publicados. Veja [33], [34], [35], [36] e [37].

Palavras-chave: problemas de valor na fronteira, grau de Leray-Schauder, operadores compactos, problemas de ponto fixo, medida de Kuratowski de não compacidade. 


\section{Abstract}

DALLOS, D. P. Existence results for some nonlinear problems of boundary value differential equations. 2017. 87 f. Tese (Doutorado) - Instituto de Matemática e Estatística, Universidade de São Paulo, São Paulo, 2017.

The main purpose of this work is to study the existence of solutions to some boundary value problems for nonlinear ordinary differential equations in finite and infinite dimension. All systems considered in this research are transformed into functional equations in which the objective is to find a fixed point of a suitable operator defined in a space of functions (which depends on the studied problem). To do this, we use the Leray-Schauder degree and a concept of topological degree due to R. Nussbaum for non-compact perturbations of identity in Banach spaces.

The new results presented in this work are contained in 5 papers, two of which have already been published. See [33], [34], [35], [36] and [37].

Keywords: boundary value problem, Leray-Schauder degree, compact operators, fixed point problems, Kuratowski's measure of non-compactness. 


\section{Sumário}

1 Introdução 1

2 Preliminares $\quad 5$

2.1 Alguns resultados clássicos . . . . . . . . . . . . . . . . . . 5

2.2 O grau de Brouwer em $\mathbb{R}^{n} \ldots \ldots \ldots \ldots \ldots \ldots$

2.3 Alguns conceitos sobre os espaços de Banach _ . . . . . . . . . . . . . 8

2.4 O grau de Leray-Schauder . . . . . . . . . . . . . . . . . . . . . . . . . . . . . . . . . . . . . . . . . . . .

2.5 O grau para funções $\alpha$-condensantes . . . . . . . . . . . . . . . . . . . . . 14

3 Problemas em dimensão finita $r$

3.1 Notações Básicas . . . . . . . . . . . . . . . . . . . . . . . . . . . . . . . . 19

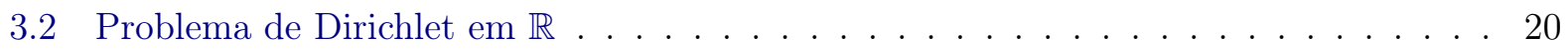

3.3 Problemas com homeomorfismos singulares em $\mathbb{R} \ldots \ldots \ldots \ldots$

3.3.1 Problemas do tipo misto com homeomorfismos singulares em $\mathbb{R}$. . . . . . . 28

3.4 Problemas com homeomorfismos limitados em $\mathbb{R} \ldots \ldots \ldots$. . . . . . . 31

3.4.1 Problemas do tipo misto com homeomorfismos limitados em $\mathbb{R}$. . . . . . . 35

3.5 Problemas do tipo misto com homeomorfismos clássicos em $\mathbb{R} \ldots$. . . . . . . . . . 37

3.6 Problemas do tipo misto generalizado em $\mathbb{R} \ldots \ldots \ldots \ldots$. . . . . . . . . . . 41

4 Problemas em dimensão infinita $\quad 53$

4.1 Notações Básicas . . . . . . . . . . . . . . . . . . . . . . . . 53

4.2 Problemas do tipo misto em espaços de Banach . . . . . . . . . . . . . . . . . . . 54

4.3 Problema de Dirichlet em espaços de Banach . . . . . . . . . . . . . . . . . 63

4.4 Problemas de tipo misto com outras condições de fronteira . . . . . . . . . . . . . . 71

$\begin{array}{lll}5 & \text { Problemas abertos } & 73\end{array}$

$\begin{array}{ll}\text { Referências Bibliográficas } & 75\end{array}$ 


\section{Capítulo 1}

\section{Introdução}

Neste trabalho, vamos estudar o problema da existência de soluções para problemas de valores de contorno não lineares do tipo

$$
\left\{\begin{array}{l}
\left(\varphi\left(u^{\prime}\right)\right)^{\prime}=f\left(t, u, u^{\prime}\right) \\
l\left(u, u^{\prime}\right)=0
\end{array}\right.
$$

onde, dado $T>0, l\left(u, u^{\prime}\right)=0$ denota condições de fronteira em $[0, T]$ que podem ser

i) do tipo periódico, $u(0)=u(T), u^{\prime}(0)=u^{\prime}(T)$,

ii) de Dirichlet, $u(0)=0=u(T)$,

iii) de Neumann, $u^{\prime}(0)=0=u^{\prime}(T)$,

iv) do tipo misto.

O operador $\varphi$ é um homeomorfismo que pode ser

1) limitado, ou seja, $\varphi: X \longrightarrow B_{a}(0) \subset X$, ou

2) singular, ou seja, $\varphi: B_{a}(0) \subset X \longrightarrow X$, ou

3) clássico, ou seja, $\varphi: X \longrightarrow X$.

Os problemas tratados nesta tese são colocados em dimensão finita e infinita. Assim, o espaço $X$ acima será simplesmente $\mathbb{R}$ em toda a primeira parte do trabalho, em dimensão finita, enquanto $X$ denotará na segunda parte do trabalho um espaço de Banach real, e os problemas tratados serão em dimensão infinita. $B_{a}(0)$ denota a bola em $X$ de centro em 0 e raio $a$. No caso em que $X=\mathbb{R}, B_{a}(0)$ é simplesmente o intervalo $(-a, a)$. Por último, $f:[0, T] \times X \times X \longrightarrow X$ é uma função contínua.

Diremos que uma função $u:[0, T] \longrightarrow X$ de classe $C^{1}$ é solução do problema $(P l)$ se

1) satisfaz as condições de fronteira,

2) é tal que a função $t \longmapsto \varphi\left(u^{\prime}(t)\right)$ está bem definida,

3) $t \longmapsto \varphi\left(u^{\prime}(t)\right)$ é de classe $C^{1}$ e satisfaz $\left(\varphi\left(u^{\prime}(t)\right)\right)^{\prime}=f\left(t, u(t), u^{\prime}(t)\right)$ para todo $t$ em $[0, T]$.

Equacões de tipo $(\mathrm{Pl})$ modelam uma ampla gama de fenômenos físicos, biológicos, químicos e econômicos, entre outros. Em particular, C. Bereanu e J. Mawhin em [6] estudam a seguinte aplicação: quando um elétron se move em altas velocidades, mas só é suavemente acelerado pelo campo de radiação e o campo de Coulomb, a dinâmica e velocidade das partículas estão determinadas por

$$
r^{\prime}(t)=\frac{p(t)}{\sqrt{1+|p(t)|^{2}}}
$$




$$
p^{\prime}(t)=-\frac{e^{2}}{h c}\left[E(t, r(t))+r^{\prime}(t) \times B(t, r(t))\right],
$$

onde $r, p: \mathbb{R} \longrightarrow \mathbb{R}^{3}$ denotam respectivamente a posição e o momento da partícula, $E, B: \mathbb{R} \times \mathbb{R}^{3} \longrightarrow$ $\mathbb{R}^{3}$ são os campos elétricos e magnéticos externos, $\times$ denota o produto vetorial em $\mathbb{R}^{3}$ e $e, h, c$ são as constantes físicas usuais. Como

$$
r^{\prime}(t)=\frac{p(t)}{\sqrt{1+|p(t)|^{2}}} \Longleftrightarrow p(t)=\frac{r^{\prime}(t)}{\sqrt{1-\left|r^{\prime}(t)\right|^{2}}},
$$

este sistema pode ser escrito como

$$
\left(\frac{r^{\prime}(t)}{\sqrt{1-\left|r^{\prime}(t)\right|^{2}}}\right)^{\prime}=-\frac{e^{2}}{h c}\left[E(t, r(t))+r^{\prime}(t) \times B(t, r(t))\right],
$$

que de fato é da forma $(P l)$

$$
\left(\varphi\left(r^{\prime}\right)\right)^{\prime}=f\left(t, r, r^{\prime}\right)
$$

com

$$
\varphi(x)=\frac{x}{\sqrt{1-|x|^{2}}} \quad\left(x \in \mathbb{R}^{3}\right) .
$$

Okrasiński e Plociniczak em [32] estudam o seguinte problema de valor na fronteira:

$$
\left\{\begin{array}{l}
-\left(\frac{u^{\prime}}{\sqrt{1+u^{\prime 2}}}\right)^{\prime}+a u=\frac{b}{\sqrt{1+u^{\prime 2}}} \\
u^{\prime}(0)=u(1)=0
\end{array}\right.
$$

para quaisquer $a>0$ e $b>0$, o qual é proposto como um modelo da forma da córnea.

Nesta investigação, abordamos vários sistemas da forma $(P l)$ dependendo da natureza de $\varphi$ e das condições de fronteira, e estabelecemos resultados que comprovam a existência de ao menos uma solução. A abordagem é topológica: $(P l)$ é transformado em um problema funcional equivalente de tipo $M(u)=u$, ou seja, um problema de ponto fixo para um operador $M$ definido em um oportuno espaço de funções. Em seguida, dependendo das condições de fronteira e de hipóteses particulares sobre $f$, obtemos estimativas a priori para as possíveis soluções, que constituem uma condição fundamental para a aplicação de teoremas de ponto fixo, do grau de Leray-Schauder ou do grau de Nussbaum. ${ }^{1}$

Destacamos aqui que esta abordagem tem uma literatura enorme. Problemas de tipo $(\mathrm{Pl})$ no espaço $\mathbb{R}^{n}$ são estudados por exemplo em artigos de Bereanu e Mawhin [8, 7, 6, 4, 5, 9], Manásevich e Mawhin [24], Bouches e Mawhin [10], Benevieri, do Ó e Souto de Medeiros [2, 3], Girg [18] entre muitos outros autores.

Nos últimos anos, os problemas de valores de contorno têm sido objeto de intensas investigações devido a duas razões principais. Uma delas consiste na grande utilidade de tais equações na modelagem de vários fenômenos que ocorrem em diversas áreas do conhecimento como, por exemplo, dinâmica populacional, engenharia, física, química, medicina e economia, entre outras. A segunda motivação é pelo interesse intrinsicamente matemático que possuem.

De acordo com os referentes teóricos, não existem métodos construtivos gerais que permitam encontrar soluções de equações diferenciais não lineares de tipo $(P l)$, de tal forma que se faz necessário um estudo tão preciso como seja possível das propriedades qualitativas destas. Nesta direção, a teoria do grau topológico tem sido desenvolvida como um método utilizado principalmente para estudar o conjunto das soluções de Equações Diferenciais Ordinárias (EDO) e Equações Diferenciais Parciais

\footnotetext{
${ }^{1}$ Ao longo deste trabalho, a notação grau de Nussbaum indicará o grau para perturbações $\alpha$-condensantes da identidade em espaços de Banach, que foi introduzido por R. Nussbaum (e F. Brouwder) no final da década de 1960.
} 
(EDP), obtendo infomações sobre a existência, multiplicidade e a natureza destas soluções.

Os métodos topológicos representam uma ferramenta importante da análise matemática que frequentemente aparecem associados a estruturas algébricas e geométricas, que permite estudar a simetria, a generalidade e aplicação de alguns problemas específicos. Um exemplo está na análise não linear, onde estes ramos da matemática são articulados para resolver equações diferenciais.

A motivação principal da nossa investigação é a tentativa de estender alguns resultados dos autores acima mencionados, abordando problemas que não parecem estar resolvidos em literatura.

No Capítulo 2 deste trabalho, apresentamos alguns resultados preliminares de Topologia geral e Análise que serão usados nos capítulos seguintes.

No Capítulo 3, estudamos os problemas de tipo $(P l)$ em dimensão finita $(X=\mathbb{R})$. Utilizamos o Teorema do ponto fixo de Schauder ou o grau topológico de Leray-Schauder para mostrar a existência de soluções.

O Capítulo 4 é dedicado à extensão em dimensão infinita de alguns dos problemas tratados no capítulo anterior. Continuamos usando a mesma abordagem topológica. Os problemas de tipo $(P l)$ são transformados em problemas funcionais não lineares de ponto fixo $u=M(u)$ por meio da introdução de oportunos operadores $M$. Dependendo das propriedades de $M$ (que pode ser completamente contínuo ou $\alpha$-condensante), resultados de existência de soluções são provados aplicando o Teorema do ponto fixo de Darbo, o grau topológico de Leray-Schauder ou o grau topológico de Nussbaum.

No último capítulo, são rapidamente apresentados alguns problemas em aberto que constituem objetos da pesquisa futura. 


\section{Capítulo 2}

\section{Preliminares}

O propósito deste capítulo é apresentar alguns resultados gerais que julgamos importantes para ajudar a compreensão deste trabalho. Em particular, na primeira seção mencionamos alguns fatos clássicos de Topologia geral e Análise. Na segunda seção enunciamos a definição de grau topológico de Brouwer e suas principais propriedades. Posteriormente, expressamos alguns conceitos básicos da teoria dos espaços de Banach, lembrando as noções de diferenciabilidade e integração em espaços de Banach. Destinamos a quarta seção para apresentar uma breve e resumida introdução sobre o grau de Leray-Schauder, ferramenta fundamental que generaliza o grau de Brouwer e permite a obtenção dos resultados referentes à existência de soluções para os problemas que estudamos nos dois próximos capítulos. Finalmente, destacamos o conceito de medida de não compacidade de Kuratowski, as definições de aplicação $\alpha$-contractiva e $\alpha$-condensante para as quais é definido um conceito de grau topológico devido a R. Nussbaum que, como já dito na introdução, será chamado grau de Nussbaum.

\subsection{Alguns resultados clássicos}

Sejam $U \subseteq \mathbb{R}^{n}$ um conjunto aberto e $f: U \longrightarrow \mathbb{R}^{m}$ uma função $C^{1}$. Dado $x \in U$, denotamos por $f^{\prime}(x)$ o diferencial de $f$ em $x$. Um elemento $x \in U$ é chamado um ponto regular de $f$ se $f^{\prime}(x)$ é sobrejetor. Caso contrário, dizemos que $x$ é um ponto crítico de $f$. Além disso, um elemento $y \in \mathbb{R}^{m}$ é chamado um valor crítico se $f^{-1}(y)$ contém pelo menos um ponto crítico, e um valor regular no caso contrário.

Observe que, se $n=m$, um ponto $x \in U$ é regular se, e somente se, o Jacobiano de $f$ em $x$, $\operatorname{det}\left(f^{\prime}(x)\right)$, é diferente de zero.

A prova do teorema a seguir pode ser encontrada por exemplo em [38].

Teorema 2.1.1 (Lema de Sard). Sejam $U \subseteq \mathbb{R}^{n}$ um conjunto aberto e $f: U \longrightarrow \mathbb{R}^{m}$ uma função de classe $C^{r}$. Se $r>\max \{0, n-m\}$, então o conjunto dos valores críticos de $f$ tem medida nula segundo Lebesgue. Em particular, o conjunto dos valores regulares de $f$ é denso em $\mathbb{R}^{m}$.

O resultado que segue é uma versão do Teorema da extensão de Tietze o qual pode ser encontrado em [15].

Teorema 2.1.2. Sejam $D \subset \mathbb{R}^{n}$ um conjunto fechado e $f: D \longrightarrow \mathbb{R}^{n}$ uma função contínua. Então, existe uma função continua $\widetilde{f}: \mathbb{R}^{n} \longrightarrow \mathbb{R}^{n}$ tal que $\widetilde{f}(x)=f(x)$ para todo $x \in D$.

Mencionamos aqui o clássico teorema da aproximação de Weierstrass (veja [15]).

Teorema 2.1.3. Sejam $D \subset \mathbb{R}^{n}$ um conjunto compacto, $f: D \longrightarrow \mathbb{R}^{n}$ uma função contínua $e$ $\epsilon>0$. Então, existe uma função de classe $C^{\infty} g: \mathbb{R}^{n} \longrightarrow \mathbb{R}^{n}$ tal que $\|f(x)-g(x)\|<\epsilon$ para todo $x \in D$.

O próximo resultado pode ser encontrado em [22]. 
Teorema 2.1.4 (Função Inversa). Sejam $U \subseteq \mathbb{R}^{n}$ um conjunto aberto e $f: U \longrightarrow \mathbb{R}^{n}$ uma função de classe $C^{r}(r \geq 1)$. Se $x_{0} \in U$ é tal que $f^{\prime}\left(x_{0}\right)$ é uma aplição invertível, então existe um conjunto aberto $V \subseteq U$ tal que a restrição $\left.f\right|_{V}$ é um difeomorfismo sobre um aberto $W$ que contém $f\left(x_{0}\right)$.

A seguir, apresentamos um resultado bem conhecido de cálculo diferencial. O leitor pode encontrar a simples demonstração em [13].

Lema 2.1.5. Sejam $\Omega \subset \mathbb{R}^{n}$ um conjunto aberto e limitado e $f: \bar{\Omega} \longrightarrow \mathbb{R}^{n}$ uma função contínua em $\bar{\Omega}$ e de classe $C^{1}$ em $\Omega$. Se y é um valor regular de $f$ em $\Omega$ e y $\notin f(\partial \Omega)$, então $f^{-1}(y) \cap \Omega$ é um conjunto finito.

\subsection{O grau de Brouwer em $\mathbb{R}^{n}$}

A teoria do grau topológico em $\mathbb{R}^{n}$ (usualmente chamado grau de Brouwer) é uma ferramenta muito importante da Análise matemática. Basicamente, o grau de Brouwer nos oferece informações sobre a existência de soluções de equações do tipo

$$
f(x)=y,
$$

onde $f: \bar{\Omega} \subset \mathbb{R}^{n} \longrightarrow \mathbb{R}^{n}$ é uma função contínua, $\Omega$ é aberto e limitado e por último, $y$ é um elemento do $\mathbb{R}^{n}$.

A teoria do grau em $\mathbb{R}^{n}$ foi primeiramente desenvolvida por Brouwer [11] em 1912. Nesta construção, o autor usa técnicas de Geometria. Daí para frente, muitos autores têm adotado diferentes tratamentos para sua elaboração. Para uma abordagem do grau de tipo topológico-algébrico veja por exemplo [16]. Uma abordagem analítica baseada no Lema de Sard pode ser encontrada por exemplo em [15]. Neste trabalho, apresentamos um resumo de sua construção analítica e exibimos suas propriedades principais. O leitor pode encontrar a construção completa do grau de Brouwer, por exemplo, em [13], [15] e [17].

Iniciamos a construção do grau de Brouwer com as seguintes definições.

Definição 2.2.1 (Terna admissível). Sejam $U$ um subconjunto qualquer do $\mathbb{R}^{n}$ e $\Omega \subset \mathbb{R}^{n}$ um conjunto aberto e limitado com $\bar{\Omega} \subseteq U$. Se $f: U \longrightarrow \mathbb{R}^{n}$ é uma função contínua em $\bar{\Omega}$ e y um elemento arbitrário do $\mathbb{R}^{n}$ tal que $y \notin f(\partial \Omega)$, então dizemos que $(f, \Omega, y)$ é uma terna admissível para o grau topológico.

Definição 2.2.2 (Terna admissível regular). Uma terna admissível $(f, \Omega, y)$ é chamada regular se $f \in C^{2}(\Omega)$ e $y$ é um valor regular de $f$ em $\Omega$.

Seja $\Delta$ o conjunto das ternas admissíveis para o grau de Brouwer. Queremos definir uma função $\operatorname{deg}_{B}: \Delta \longrightarrow \mathbb{Z}$ esperando que satisfaça as seguintes propriedades fundamentais:

1. (Normalização) Sejam $I: \mathbb{R}^{n} \longrightarrow \mathbb{R}^{n}$ a função identidade e $\Omega \subset \mathbb{R}^{n}$ um conjunto aberto limitado. Então,

$$
\operatorname{deg}_{B}(I, \Omega, y)=1, \quad \forall y \in \Omega
$$

2. (Aditividade) Seja $(f, \Omega, y)$ uma terna admissível. Se $\Omega_{1}, \Omega_{2}$ são subconjuntos abertos e disjuntos de $\Omega$ com $y \notin f\left(\bar{\Omega} \backslash\left(\Omega_{1} \cup \Omega_{2}\right)\right)$, então

$$
\operatorname{deg}_{B}(f, \Omega, y)=\operatorname{deg}_{B}\left(f, \Omega_{1}, y\right)+\operatorname{deg}_{B}\left(f, \Omega_{2}, y\right) .
$$

3. (Invariância homotópica) Sejam $\Omega \subset \mathbb{R}^{n}$ um conjunto aberto limitado, $h:[0,1] \times \bar{\Omega} \longrightarrow \mathbb{R}^{n}$ uma aplicação contínua e $y:[0,1] \longrightarrow \mathbb{R}^{n}$ uma curva contínua tais que $y(t) \notin h(t, \partial \Omega)$ para todo $t \in[0,1]$. Então, $\operatorname{deg}_{B}(h(t, \cdot), \Omega, y(t))$ é independente de $t \in[0,1]$. 
Sobre a construção do grau de Brouwer, no primeiro momento damos a definição para ternas admissíveis regulares. Em seguida, estendemos a definição do grau ao caso em que y é um valor crítico de $f$ em $\Omega$. No terceiro momento, vamos estender tal definição para ternas admissíveis gerais. Vejamos aqui rapidamente esta abordagem.

Seja $(f, \Omega, y)$ uma terna admissível regular. Então, pelo Lema 2.1.5, $f^{-1}(y) \cap \Omega$ é finito. De fato pelo Teorema da Função Inversa (Teorema 2.1.4) $f^{-1}(y) \cap \Omega$ é compacto e discreto. Isso justifica a seguinte definição do grau de Brouwer.

Definição 2.2.3. Seja $(f, \Omega, y)$ uma terna admissível regular. Então, definimos o grau de Brouwer de $(f, \Omega, y)$ como

$$
\operatorname{deg}_{B}(f, \Omega, y)=\sum_{x \in f^{-1}(y) \cap \Omega} \operatorname{sgn}\left(\operatorname{det}\left(f^{\prime}(x)\right)\right)
$$

onde $\operatorname{sgn}\left(\operatorname{det}\left(f^{\prime}(x)\right)\right)$ denota o sinal do determinante da matriz jacobiana associada a $f^{\prime}(x)$. Além disso, se $f^{-1}(y) \cap \Omega=\emptyset$, definimos

$$
\operatorname{deg}_{B}(f, \Omega, y)=0
$$

O próximo resultado, cuja prova pode ser encontrada em [13], nos permite estender esta definição para o caso em que $y$ seja um valor crítico de $f$ em $\Omega$.

Proposição 2.2.4. Sejam $(f, \Omega, y)$ uma terna admísivel com $f \in C^{2}(\Omega)$ e $y_{1}, y_{2}$ valores regulares de $f$ em $\Omega$ tais que $\left\|y-y_{i}\right\|<\operatorname{dist}(y, f(\partial \Omega)), i=1,2$. Então,

$$
\operatorname{deg}_{B}\left(f, \Omega, y_{1}\right)=\operatorname{deg}_{B}\left(f, \Omega, y_{2}\right) .
$$

Agora, estendemos a definição do grau de Brouwer para valores críticos. Denotamos

$$
\rho=\operatorname{dist}(y, f(\partial \Omega))
$$

a distância do ponto $y$ do conjunto $f(\partial \Omega)$. Ressaltamos que $\rho$ é um número positivo, isso porque $\partial \Omega$ é compacto sendo $\Omega$ limitado e portanto $f(\partial \Omega)$ é compacto e não contém $y$.

Definição 2.2.5. Seja $(f, \Omega, y)$ uma terna admissível com $f \in C^{2}(\Omega)$ e $y$ um valor crítico de $f$ em $\Omega$. Então,

$$
\operatorname{deg}_{B}(f, \Omega, y)=\operatorname{deg}_{B}\left(f, \Omega, y^{1}\right),
$$

onde $y^{1}$ é um valor regular qualquer de $f$ em $\Omega$ tal que $\left\|y-y^{1}\right\|<\rho$ e $\operatorname{deg}_{B}\left(f, \Omega, y^{1}\right)$ é dado pela Definição 2.2.3.

É importante notar que a existência do valor regular $y^{1}$ é garantida pelo Lema de Sard (Lema 2.1.1).

Finalmente, estendemos a definição do grau de Brouwer às funções contínuas. O resultado que segue pode ser encontrado em [13].

Proposição 2.2.6. Sejam $(f, \Omega, y)$ uma terna admissivel e $g_{1}, g_{2}: U \longrightarrow \mathbb{R}^{n}$ funções contínuas em $\bar{\Omega}$ e de classe $C^{2}$ em $\Omega$ tais que

$$
\sup _{x \in \bar{\Omega}}\left\|g_{i}(x)-f(x)\right\|<\rho, i=1,2 .
$$

Então,

$$
\operatorname{deg}_{B}\left(g_{1}, \Omega, y\right)=\operatorname{deg}_{B}\left(g_{2}, \Omega, y\right)
$$

É importante constatar que a existência das funções $g_{1}, g_{2}$ é garantida pelo Teorema 2.1.3.

Concluímos a construcão do grau topológico de Brouwer com a seguinte definição, justificada pela proposição anterior. 
Definição 2.2.7. Seja $(f, \Omega, y)$ uma terna admissível. Então, definimos

$$
\operatorname{deg}_{B}(f, \Omega, y)=\operatorname{deg}_{B}(g, \Omega, y)
$$

onde $g: U \longrightarrow \mathbb{R}^{n}$ é qualquer função contínua em $\bar{\Omega}$ e de classe $C^{2}$ em $\Omega$ com $\sup _{x \in \bar{\Omega}}\|g(x)-f(x)\|<\rho$ e $\operatorname{deg}_{B}(g, \Omega, y)$ é dado pela Definição 2.2.5.

As demonstrações dos resultados seguintes podem ser achadas em [13] e [15].

Teorema 2.2.8. O grau de Brouwer satisfaz as três propriedades fundamentais: Normalização, Aditividade e Invariância homotópica.

Exibimos agora uma lista de outras propriedades do grau de Brouwer que são consequências das propriedades do Teorema 2.2.8.

Proposição 2.2.9. As seguintes propriedades são válidas:

1. (Existência de solução) Seja $(f, \Omega, y)$ uma terna admissivel. Se

$$
\operatorname{deg}_{B}(f, \Omega, y) \neq 0,
$$

então $f^{-1}(y) \cap \Omega \neq \emptyset$.

2. (Propriedade do bordo) Sejam $(f, \Omega, y)$ e $(g, \Omega, y)$ ternas admissiveis. Se $f(x)=g(x)$ para todo $x \in \partial \Omega$, então

$$
\operatorname{deg}_{B}(f, \Omega, y)=\operatorname{deg}_{B}(g, \Omega, y) .
$$

3. (Função oposta) Se $(f, \Omega, y)$ for uma terna admissivel, então $(-f, \Omega,-y)$ será admissivel e

$$
\operatorname{deg}_{B}(-f, \Omega,-y)=(-1)^{n} \operatorname{deg}_{B}(f, \Omega, y) .
$$

Aqui, $n$ é a dimensão do espaço euclidiano $\mathbb{R}^{n}$.

4. (Invariância por translação) Se $(f, \Omega, y)$ for uma terna admissivel, então $(f-y, \Omega, 0)$ será admissivel $e$

$$
\operatorname{deg}_{B}(f-y, \Omega, 0)=\operatorname{deg}_{B}(f, \Omega, y)
$$

5. (Excisão) Se $(f, \Omega, y)$ é uma terna admissivel e $\Omega_{1}$ é um subconjunto aberto de $\Omega$ tal que $y \notin f\left(\bar{\Omega} \backslash \Omega_{1}\right)$, então $\left(f, \Omega_{1}, y\right)$ é admissivel $e$

$$
\operatorname{deg}_{B}(f, \Omega, y)=\operatorname{deg}_{B}\left(f, \Omega_{1}, y\right)
$$

\subsection{Alguns conceitos sobre os espaços de Banach}

Nesta seção, lembramos alguns conceitos e resultados elementares sobre os espaços de Banach que serão relevantes para os próximos capítulos. Sobre esta seção, o leitor pode consultar por exemplo [15], [19] e [41].

Ao longo deste capítulo, $(X,\|\cdot\|)$ denotará um espaço de Banach real.

Definição 2.3.1. Seja $M$ um subconjunto de $X$. Diremos que $M$ é relativamente compacto se $\bar{M}$ é um subconjunto compacto de $X$. Diremos que $M$ é precompacto ou totalmente limitado se para todo $\epsilon>0$ existe um número finito de bolas $B_{\epsilon}\left(x_{i}\right) \subset X$ tais que $M \subseteq \bigcup_{i=1}^{n} B_{\epsilon}\left(x_{i}\right)$. 
Observação 2.3.2. Em um espaço métrico completo a precompacidade é equivalente à compacidade relativa.

Lembramos que $B \subset X$ é limitado se está contido numa bola. De fato, denotamos por $d(x, y)$ a distância entre os pontos $x, y$ em $X$, e definimos o diâmetro de um conjunto limitado $B$ como o número real

$$
D(B)=\sup \{d(x, y): x, y \in B\}
$$

Definição 2.3.3. Seja $V$ um espaço vetorial. Definimos o envoltório convexo de $A \subseteq V$ como o conjunto

$$
\operatorname{conv}(A)=\left\{\sum_{i=1}^{N} \alpha_{i} x_{i}: x_{i} \in A, \alpha_{i} \in \mathbb{R}, \alpha_{i} \geq 0, \sum_{i=1}^{N} \alpha_{i}=1\right\} .
$$

Uma forma de ver o envoltório convexo de $A$ é como a interseção de todos os conjuntos convexos que contêm o conjunto $A$, ou seja, o menor (por inclusão) conjunto convexo que contém $A$.

A seguir, enunciamos um interessante espaço de Banach importante para os dois próximos capítulos. Seja $I$ um intervalo compacto da reta real. Denotamos por $C_{X}(I)$ o espaço de Banach das funções contínuas $u: I \longrightarrow X$, dotado da norma uniforme $\|\cdot\|_{\infty}$. Para $A \subseteq C_{X}(I)$, usamos a notação dada em [12]:

$$
\begin{gathered}
A(t)=\{u(t): u \in A\}, \\
A(I)=\{u(t): u \in A, t \in I\} .
\end{gathered}
$$

Definição 2.3.4. Um conjunto $H \subset C_{X}(I)$ é dito equicontínuo no ponto $t_{0} \in I$ quando para todo $\epsilon>0$, existe $\delta>0$ tal que $\left|t-t_{0}\right|<\delta$ em $I$ implica $\left\|u(t)-u\left(t_{0}\right)\right\|<\epsilon$, seja qual for $u \in H$. $H$ diz-se equicontínuo quando é equicontínuo em todos os pontos de $I$.

A demonstração do resultado seguinte pode ser encontrada em [19].

Teorema 2.3.5 (Arzelà-Ascoli). $H \subset C_{X}(I)$ é relativamente compacto se, e somente se, $H$ é equicontínuo e, para cada $t \in I, H(t)$ é relativamente compacto em $X$.

Definição 2.3.6 (Diferenciação). Seja $u: I \longrightarrow X$ uma função. Diremos que $u$ é diferenciável em $t_{0} \in I$ se o limite

$$
\lim _{h \rightarrow 0} \frac{u\left(t_{0}+h\right)-u\left(t_{0}\right)}{h},
$$

chamado a derivada de $u(t)$ em $t=t_{0}$, existe em $X$. A função $u(t)$ é diferenciável quando sua derivada existe em cada ponto de $I$. Além disso, $u^{\prime}=u^{\prime}(t)$ é uma função definida em $I$ com valores no espaço $X$.

Designamos por $C_{X}^{1}(I)$ o espaço de Banach das funções contínuamente diferenciáveis com a norma usual $\|u\|_{1}=\max \left\{\|u\|_{\infty},\left\|u^{\prime}\right\|_{\infty}\right\}$.

Algumas das regras mais básicas que possui a diferenciação e que podemos verificar de forma análoga ao caso das funções reais são as seguintes:

(a) Se $x(t)$ é uma função diferenciável em $t_{0}$, então ela é contínua em $t_{0}$.

(b) Se $x(t)=x_{0}$ é um elemento constante de $X$, então $x^{\prime}(t) \equiv 0$.

(c) Se $x(t)$ e $y(t)$ são funções diferenciáveis com valores em $X$, então $x(t)+y(t)$ é diferenciável e

$$
(x(t)+y(t))^{\prime}=x^{\prime}(t)+y^{\prime}(t) .
$$


(d) Se $x(t)$ é uma função diferenciável com valores em $X$ e $\gamma(t)$ é uma função real diferenciável, então o produto $\gamma(t) x(t)$ é uma função diferenciável com valores em $X$ e

$$
(\gamma(t) x(t))^{\prime}=\gamma^{\prime}(t) x(t)+\gamma(t) x^{\prime}(t) ;
$$

em particular, $(\lambda x(t))^{\prime}=\lambda x^{\prime}(t)$, para todo $\lambda$ constante.

(e) Se $x(t)$ é uma função diferenciável em $I$ com valores em $X$, enquanto $t(\tau)$ é uma função definida em um intervalo, com valores em $I$, diferenciável em $\tau_{0}$, então a função composta de $t(\tau)$ com $x(t)$ e indicada por $y(\tau)=x(t(\tau))$ é diferenciável em $\tau_{0} \mathrm{e}$

$$
y^{\prime}\left(\tau_{0}\right)=x^{\prime}\left(t_{0}\right) t^{\prime}\left(\tau_{0}\right) \text {, onde } t_{0}=t\left(\tau_{0}\right) .
$$

O próximo resultado pode ser achado em [19].

Teorema 2.3.7 (Valor Médio). Seja $u \in C_{X}(I)$. Denotamos $I=[0, T]$. Suponha que exista no máximo um subconjunto enumerável $\Gamma$ de $[0, T]$ tal que a derivada $u^{\prime}(t)$ existe para qualquer $t \in$ $[0, T] \backslash \Gamma$. Se existe uma constante $M \geq 0$ tal que $\left|u^{\prime}(t)\right| \leq M$ para todo $t \in[0, T] \backslash \Gamma$, então

$$
|u(T)-u(0)| \leq M T .
$$

Em particular, se $u^{\prime}(t)=0$ para todo $t \in[0, T] \backslash \Gamma$, então $u(t)$ é constante, ou seja, $u(t)=u_{0} \in X$ para todo $t \in[0, T]$.

Para finalizar esta seção, vamos definir o conceito de integral para funções $u: I \longrightarrow X$ e expressar algumas de suas propriedades.

Dada uma partição

$$
P=\left\{0=t_{0} \leq \tau_{0} \leq t_{1} \leq \tau_{1} \leq t_{2} \leq \tau_{2} \leq \ldots t_{n-1} \leq \tau_{n-1} \leq t_{n}=T\right\}
$$

de $[0, T]$, em $n$ subintervalos $\left[t_{k}, t_{k+1}\right]$ com tamanhos iguais $\Delta_{k}=T / n$, e $\tau_{k}$ um ponto entre $t_{k}$ e $t_{k+1}, k=0,1,2,3, \ldots, n-1$, definimos a soma de Riemann de $f$ relativa à partição $P$ como

$$
\sum_{k=0}^{n-1} u\left(\tau_{k}\right) \Delta_{k}=\sum_{k=0}^{n-1} u\left(\tau_{k}\right) \frac{T}{n}=\frac{T}{n} \sum_{k=0}^{n-1} u\left(\tau_{k}\right),
$$

que é obviamente um elemento do espaço $X$. Suponhamos que a soma se aproxime de um limite no espaço $X$ quando $n \longrightarrow \infty$ e sob refinamento arbitrário da partição. Então esse valor limite é o que chamamos a integral de $u(t)$ no intervalo $[0, T]$, denotada por

$$
\int_{0}^{T} u(t) d t
$$

e a função $u(t)$ é chamada integrável em $[0, T]$.

O seguinte resultado pode ser encontrado em [41].

Teorema 2.3.8. Se u(t) é uma função contínua, então u(t) é integrável em $[0, T]$.

A integral definida previamente possui as seguintes propriedades, cujas provas são facilmente verificadas.

Proposição 2.3.9. Sejam $u, v: I \longrightarrow X$ funções integráveis $e \lambda \in \mathbb{R}$.

(a) $\int_{0}^{T} \lambda u(t) d t=\lambda \int_{0}^{T} u(t) d t$.

(b) $\int_{0}^{T}(u(t)+v(t)) d t=\int_{0}^{T} u(t) d t+\int_{0}^{T} v(t) d t$.

(c) $\int_{0}^{c} u(t) d t+\int_{c}^{T} u(t) d t=\int_{0}^{T} u(t) d t$, para $0<c<T$. 
(d) $\left\|\int_{0}^{T} u(t) d t\right\| \leq \int_{0}^{T}\|u(t)\| d t$.

Seja $u: I \longrightarrow X$ uma função integrável. O valor médio de $u(t)$ em $I=[0, T]$ é dado por

$$
\frac{1}{T} \int_{0}^{T} u(t) d t .
$$

Podemos agora enunciar o Teorema do Valor Médio para Integrais. A demonstração é uma consequência imediata da definição da integral vista previamente.

Teorema 2.3.10. Seja $u: I \longrightarrow X$ uma função integrável. Então, o valor médio de $u(t)$ em I pertence ao fecho do envoltório convexo do conjunto de valores de $u(t)$ em $I$.

Calcular integrais usando somas de Riemann é um trabalho extenso e por vezes muito difícil. Felizmente, existe um argumento análogo ao considerado para o caso das funções numéricas, onde se mostra que, sob boas hipóteses, uma integral pode ser calculada muito mais facilmente com uso da antiderivação, compreendida como um processo de achar uma função conhecendo-se a sua derivada.

Teorema 2.3.11. Seja $u: I \longrightarrow X$ uma função contínua em I. Então a função

$$
F(t)=\int_{0}^{t} u(s) d s
$$

é diferenciável em I, com derivada u(t). Uma função com tal propriedade é chamada de primitiva ou antiderivada de $u(t)$.

Teorema 2.3.12. Se $F(t)$ é uma primitiva de $u(t)$ em $I$, então

$$
\int_{0}^{T} u(s) d s=F(T)-F(0) .
$$

\subsection{O grau de Leray-Schauder}

Esta seção se destina a uma breve e resumida apresentação da teoria do grau topológico de Leray-Schauder. Definimos o grau e listamos algumas de suas propriedades. Este grau topológico foi introduzido por J. Leray e J. Schauder [21] em 1934 fornecendo a primeira extensão para espaços de dimensão infinita do grau de Brouwer que resumimos anteriormente, e atualmente constitui uma ferramenta importante para assegurar a existência de soluções de alguns problemas de fronteira, como veremos posteriormente. Uma construção completa pode ser encontrada nos livros de Deimling [15], Fonseca e Gangbo [17] e Lloyd [23]. No final da seção enunciamos o Teorema do ponto fixo de Schauder. As principais referências para esta seção são [15], [17] e [23]. Na dissertação de mestrado da USP de Adriano Leandro da Costa Peixoto [13], o leitor pode encontrar uma construção do grau de Leray-Schauder didaticamente interessante.

Antes de definirmos o grau de Leray-Schauder, vamos precisar de alguns resultados e noções preliminares.

Definição 2.4.1. Sejam $X$ e $Y$ dois espaços de Banach reais e $M \subseteq X$ um conjunto qualquer. Dizemos que a função $T: M \longrightarrow Y$ é completamente contínua se

(i) $T$ é contínua;

(ii) $\overline{T(A)}$ é compacto para qualquer $A \subseteq M$ conjunto limitado.

Se, além das propriedades $(i)$ e $(i i)$, tivermos $\overline{T(M)}$ compacto, dizemos que $T$ é compacta. Finalmente diremos que a função $T: M \longrightarrow Y$ tem dimensão finita se $T(M)$ está contido num subespaço de $Y$ de dimensão finita.

Sejam $M$ e $N$ espaços métricos.

Definição 2.4.2. Sejam $P \subseteq M$ um subconjunto qualquer e $f: M \longrightarrow N$ uma função. Então, dizemos que $f$ é própria em $P$ se $f^{-1}(K) \cap P$ é compacto para todo $K$ subconjunto compacto de $N$. 
Definição 2.4.3. Considere uma função $f: M \longrightarrow N$. Dizemos que $f$ é fechada, se $f(F)$ é um subconjunto fechado em $N$ para todo $F$ fechado em $M$.

Os resultados que serão apresentados a seguir servirão de base para definirmos o grau de LeraySchauder. As demonstrações podem ser encontradas em [15].

Proposição 2.4.4. Sejam $X$ um espaço de Banach e $B \subset X$ um conjunto fechado e limitado. Considere uma função compacta $T: B \longrightarrow X$. Então

(a) A perturbação da identidade I - T é uma função própria em $B$.

(b) $I-T$ é uma função fechada.

(c) Para todo $\epsilon>0$, existe uma função (compacta) de dimensão finita $T_{\epsilon}: B \longrightarrow X$ tal que

$$
\sup _{B}\left\|T(x)-T_{\epsilon}(x)\right\| \leq \epsilon .
$$

Definição 2.4.5 (Terna admissível para o grau de Leray-Schauder). Sejam $X$ um espaço de Banach, $U \subseteq X$ um conjunto qualquer e $\Omega \subset X$ um conjunto aberto limitado tal que $\bar{\Omega} \subseteq U$. Considere uma função completamente contínua $T: U \longrightarrow X$. Se $y \in X \backslash(I-T)(\partial \Omega)$, então dizemos que $(I-T, \Omega, y)$ é uma terna admissivel para o grau de Leray-Schauder.

Seja $\Delta$ o conjunto das ternas admissíveis. Queremos definir uma função, que denotaremos por $\operatorname{deg}_{L S}: \Delta \longrightarrow \mathbb{Z}$, esperando que satisfaça as seguintes propriedades fundamentais:

1. (Normalização) Sejam $I$ a função identidade e $\Omega \subset X$ um conjunto aberto e limitado. Então,

$$
\operatorname{deg}_{L S}(I, \Omega, y)=1, \quad \forall y \in \Omega \text {. }
$$

2. (Aditividade) Seja $(I-T, \Omega, y)$ uma terna admissível. Se $\Omega_{1}, \Omega_{2}$ são subconjuntos abertos e disjuntos de $\Omega$ com $y \notin(I-T)\left(\bar{\Omega} \backslash\left(\Omega_{1} \cup \Omega_{2}\right)\right)$, então

$$
\operatorname{deg}_{L S}(I-T, \Omega, y)=\operatorname{deg}_{L S}\left(I-T, \Omega_{1}, y\right)+\operatorname{deg}_{L S}\left(I-T, \Omega_{2}, y\right) .
$$

3. (Invariância homotópica) Se $H:[0,1] \times \bar{\Omega} \longrightarrow X$ é compacta, $y:[0,1] \longrightarrow X$ é contínua e $y(t) \notin(I-H(t, \cdot))(\partial \Omega)$ para todo $t \in[0,1]$, então

$$
\operatorname{deg}_{L S}(I-H(t, \cdot), \Omega, y(t)) \text { é independente de } t \in[0,1] .
$$

Nos limitaremos a enunciar o seguinte teorema que prova a existência e unicidade do grau de Leray-Schauder e que mostra que quando tivermos uma perturbação compacta da identidade $I-T$, aproximando $T$ por uma função de dimensão finita, poderemos construir o grau de Leray-Schauder a partir do grau de Brouwer. A demonstração do teorema pode ser encontrada em [15].

Teorema 2.4.6. Seja $\Delta$ o conjunto das ternas admissiveis para o grau de Leray-Schauder. Então existe exatamente uma única função que chamamos de grau de Leray-Schauder e denotamos $\operatorname{deg}_{L S}: \Delta \longrightarrow \mathbb{Z}$ que satisfaz as propriedades fundamentais: Normalização, Aditividade e Invariância homotópica. Além disso, o grau de Leray-Schauder está relacionado com o grau de Brouwer no sentido seguinte: dado $\rho:=\operatorname{dist}(y, f(\partial \Omega))$, podemos definir

$$
\operatorname{deg}_{L S}(I-T, \Omega, y)=\operatorname{deg}_{B}\left(I-\left.T_{1}\right|_{\overline{\Omega_{1}}}, \Omega_{1}, y\right),
$$

onde $T_{1}$ é qualquer função compacta de dimensão finita tal que $\sup \left\|T(x)-T_{1}(x)\right\|<\rho, \Omega_{1}=\Omega \cap X_{1}$, $X_{1}$ é qualquer subespaço de $X$ tal que $\operatorname{dim}\left(X_{1}\right)<\infty, y \in X_{1}, T_{1}(\bar{\Omega}) \subset X_{1}$, e deg $\operatorname{de}_{B}$ e grau de Brouwer. 
As três propriedades acima são chamadas de fundamentais justamente porque garantem a existência e unicidade da função que as verifica.

Observação 2.4.7. Aclaramos também que o grau de Brouwer é único no sentido de que existe uma única função $\operatorname{deg}_{B}$ com valores em $\mathbb{Z}$ e definida no conjunto das ternas admissíveis para o grau de Brouwer que verifica as três propriedades fundamentais. A prova dessa unicidade encontra-se num clássico artigo de Amann e Weiss [1].

A seguir, listamos algumas propriedades do grau de Leray-Schauder que são consequências das três propriedades fundamentais.

Proposição 2.4.8. As seguintes propriedades são válidas:

1. (Existência de solução) Seja $(I-T, \Omega, y)$ uma terna admissível. Se

$$
\operatorname{deg}_{L S}(I-T, \Omega, y) \neq 0
$$

então existe $x \in \Omega$ tal que $(I-T)(x)=y$.

2. (Propriedade do bordo) Sejam $(I-T, \Omega, y)$ e $(I-S, \Omega, y)$ ternas admissiveis. Se $T(x)=S(x)$ para todo $x \in \partial \Omega$, então

$$
\operatorname{deg}_{L S}(I-T, \Omega, y)=\operatorname{deg}_{L S}(I-S, \Omega, y) .
$$

3. (Invariância por translação) Se $(I-T, \Omega, y)$ for uma terna admissivel, então $(I-T-y, \Omega, 0)$ será admissível e

$$
\operatorname{deg}_{L S}(I-T-y, \Omega, 0)=\operatorname{deg}_{L S}(I-T, \Omega, y) .
$$

4. (Excisão) Se $(I-T, \Omega, y)$ é uma terna admissivel e $\Omega_{1}$ é um subconjunto aberto de $\Omega$ tal que $y \notin(I-T)\left(\bar{\Omega} \backslash \Omega_{1}\right)$, então $\left(I-T, \Omega_{1}, y\right)$ é admissivel $e$

$$
\operatorname{deg}_{L S}(I-T, \Omega, y)=\operatorname{deg}_{L S}\left(I-T, \Omega_{1}, y\right) .
$$

O próximo resultado pode ser encontrado por exemplo em [13].

Teorema 2.4.9. Seja $X_{0}$ um subespaço fechado de um espaço de Banach $X$. Sejam $U \subseteq X$ um conjunto qualquer, $\Omega \subset X$ um conjunto aberto limitado tal que $\bar{\Omega} \subseteq U, T: U \longrightarrow X_{0}$ uma função completamente contínua, $y \in X_{0}$ e $y \notin(I-T)(\partial \Omega)$. Então

$$
\operatorname{deg}_{L S}(I-T, \Omega, y)=\operatorname{deg}_{L S}\left(I-\left.T\right|_{U \cap X_{0}}, \Omega \cap X_{0}, y\right) .
$$

A seguir, vamos apresentar o Teorema do ponto fixo de Schauder, cuja demonstração pode ser encontrada em [15]. Este teorema foi enunciado e provado por Schauder em 1930. Veja [39].

Teorema 2.4.10 (Ponto fixo de Schauder). Sejam $X$ um espaço de Banach, $C \subset X$ um conjunto convexo limitado, fechado e não-vazio, $T: C \longrightarrow C$ uma função compacta. Então $T$ tem um ponto fixo. 


\subsection{O grau para funções $\alpha$-condensantes}

Nesta seção, apresentamos uma extensão do grau de Leray-Schauder para uma classe de perturbações da identidade definidas em termos da medida de não compacidade de Kuratowski. Esta teoria foi desenvolvida por R. Nussbaum (parcialmente em colaboração com F. Browder) [29, 30, 31] nos anos 1969-1972. No final da seção, enunciamos alguns teoremas de ponto fixo. As principais referências são [14], [15], [19] e [20].

Primeiramente, lembramos, seguindo [19], a definição da medida de não compacidade de Kuratowski para conjuntos limitados de $X$.

Seja $B \subset X$ um conjunto limitado. Então, definimos a medida de não compacidade de Kuratowski como

$$
\alpha(B)=\inf [d>0: B \text { é coberto por um número finito de conjuntos com diâmetro } \leq d] .
$$

As principais propriedades são as seguintes:

(a) $0 \leq \alpha(B)<\infty$.

(b) $\alpha(B)=0$ se, e somente se, $\bar{B}$ é compacto.

(c) Se $S \subset B$, então $\alpha(S) \leq \alpha(B)$.

(d) $\alpha(\bar{B})=\alpha(B)$.

(e) $\alpha(B \cup S)=\max \{\alpha(B), \alpha(S)\}$.

(f) $\alpha(\lambda B)=|\lambda| \alpha(B)$, onde $\lambda \in \mathbb{R}$ e $\lambda B=\{\lambda b: b \in B\}$.

(g) $\alpha(B+S) \leq \alpha(B)+\alpha(S)$, onde $B+S=\{b+s: b \in B, s \in S\}$.

(h) $\alpha(\overline{c o n v}(B))=\alpha(B)$.

Os seguintes conceitos de função $\alpha$-condensante e $\alpha$-contractiva são baseados na noção de medida de não compacidade.

Definição 2.5.1. Sejam $X$ um espaço de Banach e $M \subseteq X$ um conjunto qualquer. Dizemos que a função $F: M \longrightarrow X$ é Lipschitz se existe uma constante $k>0$ tal que

$$
|F(x)-F(y)| \leq k|x-y|
$$

para todo $x, y \in M$. O número $k$ é chamado constante de Lipschitz para $F$. Dizemos que a função $F: M \longrightarrow X$ é uma contração estrita se $k<1$; se sua constante de Lipschitz for igual a $1, F$ será chamada não expansiva.

Definição 2.5.2. Sejam $X$ um espaço de Banach e $M \subseteq X$ um conjunto qualquer. Dizemos que a função $F: M \longrightarrow X$ é $\alpha$-Lipschitz se

(i) $F$ é contínua;

(ii) $F$ é limitada (isto é, envia conjuntos limitados em conjuntos limitados);

(iii) existe uma constante $k \geq 0$ tal que

$$
\alpha(F(B)) \leq k \alpha(B)
$$

para todo $B \subseteq M$ conjunto limitado. O número $k$ é chamado constante $\alpha$-Lipschitz para $F$.

Se a constante $\alpha$-Lipschitz é menor que 1 , então $F$ é chamada uma $\alpha$-contração, e se $k=1$, então $F$ é chamada $\alpha$-não expansiva. Se, além das propriedades $(i)$ e $(i i)$, tivermos 


$$
\alpha(F(B))<\alpha(B)
$$

se $B \subseteq M$ é limitado e $\alpha(B)>0$, dizemos que $F$ é $\alpha$-condensante.

Claramente, toda função completamente contínua é uma $\alpha$-contração e toda $\alpha$-contração é uma função $\alpha$-condensante. Além disso, se $F: M \longrightarrow X$ é Lipschitz de constante $k$, então $F$ é $\alpha$-Lipschitz com a mesma constante $k$.

A prova da proposição a seguir pode ser encontrada em [15].

Proposição 2.5.3. Sejam $X$ um espaço de Banach e $\Omega \subset X$ um conjunto aberto e limitado. Considere uma função $\alpha$-condensante $F: \bar{\Omega} \longrightarrow X$. Então a perturbação $I-F$ é uma função própria (e, portanto, fechada) em $\bar{\Omega}$.

Definição 2.5.4 (Terna admissível). Sejam $X$ um espaço de Banach e $\Omega \subset X$ um conjunto aberto e limitado. Considere uma função $\alpha$-condensante $F: \bar{\Omega} \longrightarrow X$. Se $y \in X \backslash(I-F)(\partial \Omega)$, então dizemos que $(I-F, \Omega, y)$ é uma terna admissível para o grau de Nussbaum.

Seja $\Delta$ o conjunto das ternas admissíveis. Da mesma forma que foi feito com o grau de LeraySchauder, queremos definir uma função $\operatorname{deg}_{N}: \Delta \longrightarrow \mathbb{Z}$ que vamos chamar o grau de Nussbaum esperando que satisfaça as seguintes propriedades fundamentais:

1. (Normalização) Sejam $I$ a função identidade e $\Omega \subset X$ um conjunto aberto limitado. Então

$$
\operatorname{deg}_{N}(I, \Omega, y)=1, \forall y \in \Omega \text {. }
$$

2. (Aditividade) Seja $(I-F, \Omega, y)$ uma terna admissível. Se $\Omega_{1}, \Omega_{2}$ são subconjuntos abertos e disjuntos de $\Omega$ com $y \notin(I-F)\left(\bar{\Omega} \backslash\left(\Omega_{1} \cup \Omega_{2}\right)\right)$. Então,

$$
\operatorname{deg}_{N}(I-F, \Omega, y)=\operatorname{deg}_{N}\left(I-F, \Omega_{1}, y\right)+\operatorname{deg}_{N}\left(I-F, \Omega_{2}, y\right) .
$$

3. (Invariância homotópica) Se $H:[0,1] \times \bar{\Omega} \longrightarrow X$ é contínua e $\alpha(H([0,1] \times B))<\alpha(B)$ para todo $B \subset \bar{\Omega}$ com $\alpha(B)>0, y:[0,1] \longrightarrow X$ é contínua e $y(t) \notin(I-H(t, \cdot))(\partial \Omega)$ para todo $t \in[0,1]$, então

$$
\operatorname{deg}_{N}(I-H(t, \cdot), \Omega, y(t)) \text { é independente de } t \in[0,1] .
$$

A seguir, apresentamos um importante teorema que estabelece a existência e a unicidade do grau topológico de Nussbaum, cuja demonstração pode ser vista em [15].

Teorema 2.5.5. Seja $\Delta$ o conjunto das ternas admissiveis. Então existe exatamente uma única função $\operatorname{deg}_{N}: \Delta \longrightarrow \mathbb{Z}$ satisfazendo as propriedades fundamentais de Normalização, Aditividade $e$ Invariância homotópica. Além disso, temos as seguintes afirmações:

(a) Seja $F \alpha$-contractiva e suponhamos exista $C \subset X$, conjunto convexo fechado, tal que $C_{\infty} \subseteq C$, onde $C_{\infty}=\bigcap_{n=0}^{\infty} C_{n}, C_{0}=\overline{\operatorname{conv}}(F(\bar{\Omega})+y), C_{n}=\overline{\operatorname{conv}}\left(F\left(\bar{\Omega} \cap C_{n-1}\right)+y\right)$ para $n \geq 1$. Suponhamos também que $F(\bar{\Omega} \cap C)+y \subseteq C$ e que $F(\bar{\Omega} \cap C)$ seja relativamente compacto. Então

$$
\operatorname{deg}_{N}(I-F, \Omega, y)=\operatorname{deg}_{L S}\left(I-F R, R^{-1}(\Omega) \cap \Omega, y\right)
$$

onde $R$ é qualquer retração de $X$ sobre $C$. Em particular, se $C_{\infty} \neq \emptyset$, então $C=C_{\infty}$. Se não existe tal conjunto $C$, então $\operatorname{deg}_{N}(I-F, \Omega, y)=0$.

(b) Se $F$ é $\alpha$-condensante e não é $\alpha$-contractiva, então 


$$
\operatorname{deg}_{N}(I-F, \Omega, y)=\operatorname{deg}_{N}(I-k F, \Omega, y)
$$

onde $k \in[0,1),(1-k) \sup _{\bar{\Omega}}\|F(x)\|<\rho(y,(I-F)(\partial \Omega))$ e $\rho$, analogamente às seçôes anteriores, denota a distância entre y e $(I-F)(\partial \Omega)$.

As propriedades apresentadas a seguir são análogas às vistas para os graus de Brouwer e LeraySchauder. As demonstrações podem ser encontradas em [15].

Proposição 2.5.6. As seguintes propriedades são válidas:

1. (Existência de solução) Seja $(I-F, \Omega, y)$ uma terna admissivel. Se

$$
\operatorname{deg}_{N}(I-F, \Omega, y) \neq 0
$$

então existe $x \in \Omega$ tal que $(I-F)(x)=y$.

2. (Propriedade do bordo) Sejam $(I-F, \Omega, y)$ e $(I-S, \Omega, y)$ ternas admissiveis. Se $F(x)=S(x)$ para todo $x \in \partial \Omega$. Então,

$$
\operatorname{deg}_{N}(I-F, \Omega, y)=\operatorname{deg}_{N}(I-S, \Omega, y) .
$$

3. (Excisão) Se $(I-F, \Omega, y)$ é uma terna admissivel e $\Omega_{1}$ um subconjunto aberto de $\Omega$ tal que $y \notin(I-F)\left(\bar{\Omega} \backslash \Omega_{1}\right)$, então $\left(I-F, \Omega_{1}, y\right)$ é admissivel $e$

$$
\operatorname{deg}_{N}(I-F, \Omega, y)=\operatorname{deg}_{N}\left(I-F, \Omega_{1}, y\right) .
$$

A seguir, apresentamos alguns resultados que são de muita importância para os dois próximos capítulos. Ao leitor que deseje verificar as demonstrações recomendamos a leitura de [19].

Denotamos por $\alpha, \alpha_{c}$ e $\alpha_{1}$ as medidas de não compacidade de Kuratowski em $X, C_{X}(I)$ e $C_{X}^{1}(I)$, respectivamente. Lembramos que para $I=[0, T]$, designamos por $C_{X}(I)$ o espaço de Banach das funções contínuas $u: I \longrightarrow X$, dotado da norma uniforme $\|\cdot\|_{\infty}$ e $C_{X}^{1}(I)$ o espaço de Banach das funções contínuamente diferenciáveis com a norma usual $\|u\|_{1}=\max \left\{\|u\|_{\infty},\left\|u^{\prime}\right\|_{\infty}\right\}$.

Teorema 2.5.7. Seja A um subconjunto limitado e equicontínuo de $C_{X}(I)$. Então $\alpha(A(t))$ é contínu em $I$.

Teorema 2.5.8. Se A é um subconjunto limitado e equicontínuo de $C_{X}(I)$, então os seguintes enunciados são válidos:

(1) $\alpha_{c}(A)=\alpha(A(I))$.

(2) $\alpha(A(I))=\max _{I} \alpha(A(t))$.

Para $A \subseteq C_{X}^{1}(I)$, denotamos

$$
\begin{gathered}
A^{\prime}(t)=\left\{u^{\prime}(t): u \in A\right\}, \\
A^{\prime}=\left\{u^{\prime}: u \in A\right\}, \\
A^{\prime}(I)=\left\{u^{\prime}(t): u \in A, t \in I\right\} .
\end{gathered}
$$

Lema 2.5.9. Se $S$ é um subconjunto limitado de $\mathbb{R}$ e $B$ é um subconjunto limitado de $X$, então

$$
\alpha(S B)=\left(\sup _{S}|t|\right) \alpha(B)
$$

onde $S B=\{s b: s \in S, b \in B\}$.

Lema 2.5.10. Sejam $A, B$ subconjuntos limitados dos espaços de Banach $X$ e $Y$ respectivamente. Colocando a norma em $X \times Y$ 


$$
\|(x, y)\|=\max \{\|x\|,\|y\|\}
$$

então

$$
\alpha(A \times B)=\max \{\alpha(A), \alpha(B)\} .
$$

Lema 2.5.11. Se A é um subconjunto limitado de $C_{X}^{1}(I)$, então

(i) $\alpha_{1}(A) \geq \max \left\{\max _{I} \alpha(A(t)), \max _{I} \alpha\left(A^{\prime}(t)\right)\right\}$.

(ii) $\alpha_{1}(A) \geq \alpha(A(I))$.

(iii) $2 \alpha_{1}(A) \geq \alpha\left(A^{\prime}(I)\right)$.

Lema 2.5.12. Se $A$ é um subconjunto limitado de $C_{X}^{1}(I)$ e $A^{\prime}$ equicontínuo, então

$$
\alpha_{1}(A)=\max \left\{\max _{I} \alpha(A(t)), \max _{I} \alpha\left(A^{\prime}(t)\right)\right\} .
$$

Teorema 2.5.13. Um subconjunto $A$ de $C_{X}^{1}(I)$ é relativamente compacto se, e somente se,

(1) $A^{\prime}$ é equicontínuo e, para qualquer $t \in I, A^{\prime}(t)$ é um conjunto relativamente compacto em $X$,

(2) existe um $t_{0} \in I$ tal que $A\left(t_{0}\right)$ é um conjunto relativamente compacto em $X$.

Finalmente, vamos concluir esta seção apresentando alguns teoremas de ponto fixo, cujas demonstrações podem ser encontradas em [19].

Teorema 2.5.14 (Sadovskii). Sejam $X$ um espaço de Banach, $D \subset X$ um conjunto convexo limitado, fechado e não vazio. Se a função $T: D \longrightarrow D$ é $\alpha$-condensante, então $T$ tem um ponto fixo.

Teorema 2.5.15 (Darbo). Sejam $X$ um espaço de Banach, $D \subset X$ um conjunto convexo limitado, fechado e não vazio. Se a função $T: D \longrightarrow D$ é $\alpha$-contractiva, então $T$ tem um ponto fixo. 


\section{Capítulo 3}

\section{Problemas em dimensão finita}

Neste capítulo, estudamos problemas de tipo

$$
\left\{\begin{array}{l}
\left(\varphi\left(u^{\prime}\right)\right)^{\prime}=f\left(t, u, u^{\prime}\right) \\
l\left(u, u^{\prime}\right)=0,
\end{array}\right.
$$

onde as hipóteses sobre $f$ e $\varphi$ serão esclarecidas nas próximas seções. Estabelecemos condições que garantem existência de soluções para uma classe de equações diferenciais não lineares sujeitas a condições de contorno. Apresentamos seis seções. Na primeira introduzimos alguns conceitos e notações básicos que serão relevantes para este capítulo. Posteriormente, abordamos um problema de Dirichlet em $\mathbb{R}$, generalizando alguns resultados alcançados por C. Bereanu e J. Mawhin em [8]. Na terceira e quarta seções, estudamos sistemas com condições de fronteira de tipo misto e homeomorfismos singulares (na terceira) e limitados (na quarta), ou seja, $\varphi:(-a, a) \longrightarrow \mathbb{R}$ e $\varphi: \mathbb{R} \longrightarrow(-a, a)$, respectivamente. Tais problemas não encontramos na literatura. Na quinta seção, estamos interessados em provar a existência de pelo menos uma solução para problemas de valor de contorno do tipo misto e homeomorfismos clássicos $(\varphi: \mathbb{R} \longrightarrow \mathbb{R})$. Na última seção, mostramos a existência de soluções com condições de contorno do tipo misto generalizado que aparentemente não estão na literatura.

\subsection{Notações Básicas}

Para $T$ fixado, denotamos a norma usual em $L^{1}=L^{1}([0, T], \mathbb{R})$ por $\|\cdot\|_{L^{1}}$. Por $C=C([0, T], \mathbb{R})$ indicamos o espaço de Banach das funções reais contínuas sobre $[0, T]$, dotado da norma uniforme $\|\cdot\|_{\infty}$, por $C^{1}=C^{1}([0, T], \mathbb{R})$ o espaço de Banach das funções contínuamente diferenciáveis sobre $[0, T]$ com a norma usual $\|u\|_{1}=\|u\|_{\infty}+\left\|u^{\prime}\right\|_{\infty}$ e por $C_{0}^{1}$ o subespaço fechado de $C^{1}$ definido por $C_{0}^{1}=\left\{u \in C^{1}: u(T)=0=u(0)\right\}$. Consideramos também as seguintes aplicações:

o operador de Nemytskii $N_{f}: C^{1} \longrightarrow C$

$$
N_{f}(u)(t)=f\left(t, u(t), u^{\prime}(t)\right),
$$

onde $f:[0, T] \times \mathbb{R} \times \mathbb{R} \longrightarrow \mathbb{R}$ é uma função contínua

o operador integração $H: C \longrightarrow C^{1}$,

$$
H(u)(t)=\int_{0}^{t} u(s) d s
$$

as seguintes aplicações lineares contínuas:

$$
\begin{gathered}
K: C \longrightarrow C^{1}, \quad K(u)(t)=-\int_{t}^{T} u(s) d s \\
Q: C \longrightarrow C, \quad Q(u)(t)=\frac{1}{T} \int_{0}^{T} u(s) d s \\
S: C \longrightarrow C, \quad S(u)(t)=u(T)
\end{gathered}
$$




$$
P: C \longrightarrow C, P(u)(t)=u(0) .
$$

Observe que, de fato, $Q, S$ e $P$ são aplicações que associam um número a cada função.

Se $u \in C$, denotamos

$$
u_{m}=\min _{[0, T]} u, \quad u_{M}=\max _{[0, T]} u, \quad u^{+}=\max \{u, 0\}, \quad u^{-}=\max \{-u, 0\} .
$$

\subsection{Problema de Dirichlet em $\mathbb{R}$}

Consideremos o problema de Dirichlet

$$
\left\{\begin{array}{l}
\left(\varphi\left(u^{\prime}\right)\right)^{\prime}=f\left(t, u, u^{\prime}\right) \\
u(0)=0=u(T),
\end{array}\right.
$$

onde $\varphi: \mathbb{R} \longrightarrow(-a, a)$ é um homeomorfismo tal que $\varphi(0)=0$ e $f:[0, T] \times \mathbb{R} \times \mathbb{R} \longrightarrow \mathbb{R}$ é uma função contínua.

Entendemos por solução do problema (3.1) uma função $u:[0, T] \longrightarrow \mathbb{R}$ de classe $C^{1}$ satisfazendo as condições de fronteira e tal que a função $\varphi\left(u^{\prime}\right)$ seja de classe $C^{1}$ e satisfaça $\left(\varphi\left(u^{\prime}(t)\right)\right)^{\prime}=$ $f\left(t, u(t), u^{\prime}(t)\right)$ para todo $t$ em $[0, T]$.

Este sistema foi estudado por Bereanu e Mawhin no artigo [8]. Naquele trabalho, os autores convertem (3.1) em um problema de ponto fixo em oportunos espaços de funções. Em seguida, sob certas condições, usam o grau topológico de Leray-Schauder para demonstrar a existência de soluções. Vamos aqui apresentar este resultado.

Teorema 3.2.1 (Bereanu e Mawhin). Se $f$ satisfaz a seguinte condição

$$
\exists c>0 \text { tal que }|f(t, x, y)| \leq c<\frac{a}{2 T}, \quad \forall(t, x, y) \in[0, T] \times \mathbb{R} \times \mathbb{R},
$$

o problema (3.1) tem pelo menos uma solução.

Apresentamos aqui as ideias principais da demonstração, que se encontram em [8] (veja-se outros trabalhos, por exemplo [24]). A técnica de Bereanu e Mawhin será usada nesta tese para provar uma extensão do teorema acima.

Primeiramente, enunciamos um importante lema, cuja demonstração pode ser encontrada em [8], [7], [4] e [5]. Contudo, apresentamos aqui a prova, para melhor compreensão do desenvolvimento da nossa pesquisa.

Lema 3.2.2. Seja $B=\left\{h \in C:\|h\|_{\infty}<a / 2\right\}$. Para cada $h \in B$, existe um único número real $Q_{\varphi}=Q_{\varphi}(h) \in \operatorname{Im}(h)$ (onde $\operatorname{Im}(h)$ denota a imagem de $h$ ) tal que

$$
\int_{0}^{T} \varphi^{-1}\left(h(t)-Q_{\varphi}(h)\right) d t=0 .
$$

Além disso, a função $Q_{\varphi}: B \longrightarrow \mathbb{R}$ é contínua e a imagem de $Q_{\varphi}$ é limitada.

Demonstração. Seja $h \in B$ fixada. Definimos a aplicação contínua $G_{h}:\left[h_{m}, h_{M}\right] \longrightarrow \mathbb{R}$ por

$$
G_{h}(s)=\int_{0}^{T} \varphi^{-1}(h(t)-s) d t .
$$

Vejamos que a equação

$$
G_{h}(s)=0
$$

possui uma única solução $Q_{\varphi}(h)$. Sejam $r, s \in\left[h_{m}, h_{M}\right]$ números reais tais que

$$
\int_{0}^{T} \varphi^{-1}(h(t)-r) d t=0, \int_{0}^{T} \varphi^{-1}(h(t)-s) d t=0,
$$

ou seja,

$$
\int_{0}^{T} \varphi^{-1}(h(t)-r) d t=\int_{0}^{T} \varphi^{-1}(h(t)-s) d t
$$


Daí, segue-se que existe $\tau \in[0, T]$ tal que

$$
\varphi^{-1}(h(\tau)-r)=\varphi^{-1}(h(\tau)-s) .
$$

Usando o fato de que $\varphi^{-1}$ é injetora, temos $r=s$. Desta forma, se (3.2) tem solução, ela é única. Agora vamos provar a existência. Como $\varphi^{-1}$ é estritamente monótona e $\varphi^{-1}(0)=0$, então

$$
G_{h}\left(h_{m}\right) G_{h}\left(h_{M}\right) \leq 0 .
$$

Portanto, existe $s \in\left[h_{m}, h_{M}\right]$ tal que $G_{h}(s)=0$. Por conseguinte, para cada $h \in B$, a equação

$$
G_{h}(s)=0
$$

tem solução. E, como já vimos, é única. Desta forma, definimos a função $Q_{\varphi}: B \longrightarrow \mathbb{R}$ que satisfaz

$$
\int_{0}^{T} \varphi^{-1}\left(h(t)-Q_{\varphi}(h)\right) d t=0 .
$$

Agora vamos mostrar que a imagem de $Q_{\varphi}: B \longrightarrow \mathbb{R}$ é limitada. Para tanto, seja $h \in B$. Então

$$
\left|Q_{\varphi}(h)\right| \leq\|h\|_{\infty}<a / 2 .
$$

Portanto, a imagem de $Q_{\varphi}$ se torna limitada.

Para finalizar, vamos mostrar que $Q_{\varphi}$ é contínua. Seja $\left(h_{n}\right) \subseteq B$ uma sequência tal que

$$
\lim _{n \rightarrow \infty} h_{n}=h \in B \text {. }
$$

Como a função $Q_{\varphi}$ é limitada, então a sequência $\left(Q_{\varphi}\left(h_{n}\right)\right)$ é limitada. Portanto, há uma subsequência convergente para um certo $\widetilde{a} \in \mathbb{R}$. Sem perda de generalidade, podemos escrever

$$
\lim _{n \rightarrow \infty} Q_{\varphi}\left(h_{n}\right)=\widetilde{a} .
$$

Temos, para cada $n \in \mathbb{N}$,

$$
\int_{0}^{T} \varphi^{-1}\left(h_{n}(t)-Q_{\varphi}\left(h_{n}\right)\right) d t=0
$$

Portanto, usando o Teorema da convergência dominada, obtemos

$$
\int_{0}^{T} \varphi^{-1}(h(t)-\widetilde{a}) d t=0 .
$$

Assim, $Q_{\varphi}(h)=\widetilde{a}$, o que mostra que $Q_{\varphi}$ é contínua.

Voltamos agora ao sistema (3.1) e introduzimos uma família de sistemas dependendo de um parâmetro real $\lambda$. Para $\lambda \in[0,1]$, consideramos a família de problemas de valor na fronteira

$$
\left\{\begin{array}{l}
\left(\varphi\left(u^{\prime}\right)\right)^{\prime}=\lambda f\left(t, u, u^{\prime}\right) \\
u(0)=0=u(T) .
\end{array}\right.
$$

Seja

$$
\Omega=\left\{(\lambda, u) \in[0,1] \times C_{0}^{1}:\left\|\lambda H\left(N_{f}(u)\right)\right\|_{\infty}<a / 2\right\} .
$$

É claro que $\Omega$ é aberto em $[0,1] \times C_{0}^{1}$, e é não vazio porque $\{0\} \times C_{0}^{1} \subset \Omega$. Assim, usando o Lema 3.2 .2 , podemos definir o seguinte operador $M: \Omega \longrightarrow C_{0}^{1}$ :

$$
M(\lambda, u)=H\left(\varphi^{-1}\left[\lambda H\left(N_{f}(u)\right)-Q_{\varphi}\left(\lambda H\left(N_{f}(u)\right)\right)\right]\right) .
$$

Denotamos por $B_{a}(0)$ a bola em $C$ de centro em 0 e raio $a, \varphi^{-1}$ acima é entendido, com abuso de notação, como o operador $\varphi^{-1}: B_{a}(0) \longrightarrow C$ definido por $\varphi^{-1}(v)(t)=\varphi^{-1}(v(t))$. É claro que $\varphi^{-1}$ é contínuo. 
Lema 3.2.3. O operador $M$ está bem definido e é contínuo. Além disso, se $(\lambda, u) \in \Omega$ é tal que $M(\lambda, u)=u$, então u é solução de (3.5), relativa ao mesmo $\lambda$.

Demonstração. Seja $(\lambda, u) \in \Omega$. A continuidade de $M(\lambda, u)$ segue do fato de que se trata de uma composição de funções contínuas. Além disso, temos

$$
(M(\lambda, u))^{\prime}(t)=\varphi^{-1}\left[\lambda H\left(N_{f}(u)\right)-Q_{\varphi}\left(\lambda H\left(N_{f}(u)\right)\right)\right](t),
$$

também que é uma composição de funções contínuas e, portanto, é contínua.

Por outro lado, usando o Lema 3.2.2, obtemos

$$
M(\lambda, u)(0)=0=M(\lambda, u)(T) .
$$

Portanto, $M(\Omega) \subset C_{0}^{1}$, o que significa que $M$ está bem definido. A continuidade de $M: \Omega \longrightarrow C_{0}^{1}$ é devida à continuidade das funções que a compõem.

Agora, seja $(\lambda, u) \in \Omega$ tal que $M(\lambda, u)=u$. Então $u$ satisfaz

$$
u(t)=M(\lambda, u)(t)=H\left(\varphi^{-1}\left[\lambda H\left(N_{f}(u)\right)-Q_{\varphi}\left(\lambda H\left(N_{f}(u)\right)\right)\right]\right)(t),
$$

para todo $t \in[0, T]$. Derivando, obtemos

$$
u^{\prime}(t)=\varphi^{-1}\left[\lambda H\left(N_{f}(u)\right)-Q_{\varphi}\left(\lambda H\left(N_{f}(u)\right)\right)\right](t) .
$$

Aplicando a função $\varphi$, segue que

$$
\varphi\left(u^{\prime}(t)\right)=\lambda H\left(N_{f}(u)\right)(t)-Q_{\varphi}\left(\lambda H\left(N_{f}(u)\right)\right) .
$$

Agora, derive novamente e conclua que

$$
\left(\varphi\left(u^{\prime}(t)\right)\right)^{\prime}=\lambda N_{f}(u)(t)
$$

para todo $t \in[0, T]$. Logo, $u$ é solução de (3.5).

Observação 3.2.4. A recíproca do lema anterior não sabemos se é válida devido à natureza de $\varphi$, pois não podemos garantir que $\| \lambda H\left(N_{f}(u) \|_{\infty}<a / 2\right.$ onde $u$ é uma solução de (3.5).

Antes de provarmos o principal resultado desta seção, apresentamos os dois seguintes lemas. O primeiro é para a obtenção de estimativas a priori para os possíveis pontos fixos de $M$. Devido à dependência de $M$ de $(\lambda, u)$ a expressão ponto fixo é aqui usada com um pequeno abuso de notação que o leitor certamente vai entender. O segundo lema mostra que o operador $M$ sob certo domínio tem propriedades importantes.

Destacamos que nosso lema seguinte generaliza a condição sobre $f$ do Teorema 3.2.1.

Lema 3.2.5. Sejam $h \in C\left([0, T], \mathbb{R}^{+}\right), n \in C^{1}(\mathbb{R}, \mathbb{R})$ e $f$ tais que

$$
\|h\|_{L^{1}}<a / 2, \varphi(y) n^{\prime}(x) y \geq 0, n(0)=0,
$$

$e$

$$
|f(t, x, y)| \leq f(t, x, y) n(x)+h(t)
$$

para todo $x, y \in \mathbb{R}$ e todo $t \in[0, T]$. Se $(\lambda, u) \in \Omega$ é tal que $M(\lambda, u)=u$, então

$$
\left\|\lambda H\left(N_{f}(u)\right)\right\|_{\infty} \leq\|h\|_{L^{1}},\left\|u^{\prime}\right\|_{\infty} \leq L \quad e \quad\|u\|_{1} \leq L+L T,
$$

onde $L=\max \left\{\left|\varphi^{-1}\left(-2\|h\|_{L^{1}}\right)\right|,\left|\varphi^{-1}\left(2\|h\|_{L^{1}}\right)\right|\right\}$.

Demonstração. Seja $(\lambda, u) \in \Omega, \operatorname{com} \lambda \neq 0$ tal que $M(\lambda, u)=u$. Então pelo Lema 3.2.3, $u$ é solução de (3.5), portanto

$$
\varphi\left(u^{\prime}\right)=\lambda H\left(N_{f}(u)\right)-Q_{\varphi}\left(\lambda H\left(N_{f}(u)\right)\right), u(0)=0=u(T),
$$


onde, para todo $t \in[0, T]$, temos

$$
\begin{aligned}
\left|\lambda H\left(N_{f}(u)\right)(t)\right| & \leq \int_{0}^{T}\left|f\left(s, u(s), u^{\prime}(s)\right)\right| d s \\
& \leq \int_{0}^{T} f\left(s, u(s), u^{\prime}(s)\right) n(u(s)) d s+\int_{0}^{T} h(s) d s .
\end{aligned}
$$

Por outro lado, como $\varphi$ é um homeomorfismo tal que

$$
\varphi(y) n^{\prime}(x) y \geq 0
$$

para todo $x, y \in \mathbb{R}, \log$,

$$
\left.\varphi\left(u^{\prime}(t)\right)\right) n^{\prime}(u(t)) u^{\prime}(t) \geq 0
$$

para todo $t \in[0, T]$ e, por conseguinte,

$$
-\int_{0}^{T} \varphi\left(u^{\prime}(t)\right) n^{\prime}(u(t)) u^{\prime}(t) d t \leq 0 .
$$

Usando a integração por partes, as condições iniciais e o fato de que $n(0)=0$, deduzimos a igualdade

$$
\int_{0}^{T}\left(\varphi\left(u^{\prime}(t)\right)\right)^{\prime} n(u(t)) d t=-\int_{0}^{T} \varphi\left(u^{\prime}(t)\right) n^{\prime}(u(t)) u^{\prime}(t) d t \leq 0 \text { para todo } t \in[0, T] .
$$

Como $\lambda \in(0,1]$ e $u$ é solução de (3.5), obtemos

$$
\int_{0}^{T} f\left(t, u(t), u^{\prime}(t)\right) n(u(t)) d t \leq 0
$$

e, portanto,

$$
\left|\lambda H\left(N_{f}(u)\right)(t)\right| \leq\|h\|_{L^{1}} .
$$

Por outro lado, como $Q_{\varphi}\left(\lambda H\left(N_{f}(u)\right)\right)$ pertence à imagem de $\lambda H\left(N_{f}(u)\right)$, obtemos que

$$
\left|\varphi\left(u^{\prime}(t)\right)\right| \leq 2\|h\|_{L^{1}}
$$

para todo $t \in[0, T]$. Assim,

$$
\left\|\varphi\left(u^{\prime}\right)\right\|_{\infty} \leq 2\|h\|_{L^{1}}
$$

Disto, concluimos que $\left\|u^{\prime}\right\|_{\infty} \leq L$, onde $L=\max \left\{\left|\varphi^{-1}\left(-2\|h\|_{L^{1}}\right)\right|,\left|\varphi^{-1}\left(2\|h\|_{L^{1}}\right)\right|\right\}$. Usando novamente as condições iniciais, temos que, para cada $t \in[0, T]$,

$$
|u(t)| \leq \int_{0}^{t}\left|u^{\prime}(s)\right| d s \leq \int_{0}^{T}\left|u^{\prime}(s)\right| d s \leq L T .
$$

Assim,

$$
\|u\|_{1} \leq L+L T
$$

Por último, se $u=M(0, u)$, então $u=0$, finalizando a demonstração .

Considerando $\rho, \kappa \in \mathbb{R}$ tais que $\|h\|_{L^{1}}<\kappa<a / 2, \rho>L+L T$, então definimos o seguinte conjunto

$$
V=\left\{(\lambda, u) \in[0,1] \times C_{0}^{1}:\left\|\lambda H\left(N_{f}(u)\right)\right\|_{\infty}<\kappa,\|u\|_{1}<\rho\right\} .
$$

Por sua definição, o conjunto $V$ é não vazio, pois $\{0\} \times\left\{u \in C_{0}^{1}:\|u\|_{1}<\rho\right\} \subset V$, é aberto e limitado em $[0,1] \times C_{0}^{1}$ e $\bar{V} \subset \Omega$. Além disso, usando os Lemas 3.2.3 e 3.2.5, deduzimos que o operador $M: \bar{V} \longrightarrow C_{0}^{1}$ está bem definido, contínuo e

$$
u \neq M(\lambda, u) \text { para todo }(\lambda, u) \in \partial V .
$$

Lema 3.2.6. O operador $M: \bar{V} \longrightarrow C_{0}^{1}$ é completamente contínuo. 
Demonstração. Seja $\Lambda$ um conjunto (limitado) de $\bar{V}$. Então, se $(\lambda, u) \in \Lambda$, existe $\delta>0$ tal que

$$
\|(\lambda, u)\|=\max \left\{\|\lambda\|,\|u\|_{1}\right\} \leq \delta .
$$

Queremos mostrar que $\overline{M(\Lambda)} \subset C_{0}^{1}$ é um conjunto compacto. Para tanto, tome $\left(v_{n}\right)$ uma sequência em $M(\Lambda)$ e $\left(\lambda_{n}, u_{n}\right)$ uma sequência em $\Lambda$ tal que $v_{n}=M\left(\lambda_{n}, u_{n}\right)$. Da fórmula (3.7), deduzimos que existe $\beta>0$ tal que

$$
\left\|N_{f}\left(u_{n}\right)\right\|_{\infty} \leq \beta
$$

para todo $n \in \mathbb{N}$.

Por outro lado, como $\left\|\lambda_{n} H\left(N_{f}\left(u_{n}\right)\right)\right\|_{\infty}<\kappa<a / 2$ para todo $n \in \mathbb{N}$, então

$$
\left\|\lambda_{n} H\left(N_{f}\left(u_{n}\right)\right)-Q_{\varphi}\left(\lambda_{n} H\left(N_{f}\left(u_{n}\right)\right)\right)\right\|_{\infty}<2 \kappa .
$$

Portanto, a sequência $\left(\lambda_{n} H\left(N_{f}\left(u_{n}\right)\right)-Q_{\varphi}\left(\lambda_{n} H\left(N_{f}\left(u_{n}\right)\right)\right)\right)$ é limitada em $C$. Além disso, para todo $n \in \mathbb{N}$ e para $t, t_{1} \in[0, T]$, segue que

$$
\begin{aligned}
& \left|\lambda_{n} H\left(N_{f}\left(u_{n}\right)\right)(t)-Q_{\varphi}\left(\lambda_{n} H\left(N_{f}\left(u_{n}\right)\right)\right)-\lambda_{n} H\left(N_{f}\left(u_{n}\right)\right)\left(t_{1}\right)+Q_{\varphi}\left(\lambda_{n} H\left(N_{f}\left(u_{n}\right)\right)\right)\right| \\
& \leq\left|\int_{0}^{t} f\left(s, u_{n}(s), u_{n}^{\prime}(s)\right) d s-\int_{0}^{t_{1}} f\left(s, u_{n}(s), u_{n}^{\prime}(s)\right) d s\right| \\
& \leq \int_{t_{1}}^{t}\left|f\left(s, u_{n}(s), u_{n}^{\prime}(s)\right)\right| d s \\
& \leq \beta\left|t-t_{1}\right|
\end{aligned}
$$

o que implica que a sequência $\left(\lambda_{n} H\left(N_{f}\left(u_{n}\right)\right)-Q_{\varphi}\left(\lambda_{n} H\left(N_{f}\left(u_{n}\right)\right)\right)\right)$ é equicontínua. Assim, usando o Teorema de Arzelà-Ascoli (Teorema 2.3.5), $\left(\lambda_{n} H\left(N_{f}\left(u_{n}\right)\right)-Q_{\varphi}\left(\lambda_{n} H\left(N_{f}\left(u_{n}\right)\right)\right)\right)$ possui uma subsequência convergente em $C$, que vamos chamar de

$$
\left(\lambda_{n_{j}} H\left(N_{f}\left(u_{n_{j}}\right)\right)-Q_{\varphi}\left(\lambda_{n_{j}} H\left(N_{f}\left(u_{n_{j}}\right)\right)\right)\right) .
$$

Sabendo que $\varphi^{-1}: B_{a}(0) \subset C \longrightarrow C$ é contínua e que

$$
M\left(\lambda_{n_{j}}, u_{n_{j}}\right)^{\prime}=\varphi^{-1}\left(\lambda_{n_{j}} H\left(N_{f}\left(u_{n_{j}}\right)\right)-Q_{\varphi}\left(\lambda_{n_{j}} H\left(N_{f}\left(u_{n_{j}}\right)\right)\right)\right),
$$

segue que a sequência $\left(M\left(\lambda_{n_{j}}, u_{n_{j}}\right)^{\prime}\right)$ é convergente em $C$ e, portanto, $\left(v_{n_{j}}\right)=\left(M\left(\lambda_{n_{j}}, u_{n_{j}}\right)\right)$ é convergente em $C_{0}^{1}$. Para finalizar a prova, suponha $\left(v_{n}\right) \subseteq \overline{M(\Lambda)}$. Seja $\left(z_{n}\right) \subseteq M(\Lambda)$ tal que

$$
\lim _{n \rightarrow \infty}\left\|z_{n}-v_{n}\right\|_{1}=0
$$

Seja, de acordo com a primeira parte da demonstração, $\left(z_{n_{j}}\right)$ uma subsequência de $\left(z_{n}\right)$ que converge para um elemento $z$. Segue que $z \in \overline{M(\Lambda)}$ e $\left(v_{n_{j}}\right)$ converge para $z$. Portanto, o resultado está provado.

Para mostrar a existência de soluções de (3.1), precisamos da seguinte propriedade de invariância homotópica estendida do grau de Leray-Schauder cuja demonstração é dada em [25].

Proposição 3.2.7. Seja $X$ um espaço de Banach, $V \subset[0,1] \times X$ aberto limitado e seja $M$ um operador completamente contínuo sobre $\bar{V}$ tal que $x \neq M(\lambda, x)$ para todo $(\lambda, x) \in \partial V$. Então o grau de Leray-Schauder

$$
\operatorname{deg}_{L S}\left(I-M(\lambda, .), V_{\lambda}, 0\right)
$$

está bem definido e independente de $\lambda$ em $[0,1]$, onde $V_{\lambda}$ é aberto, limitado (possivelmente vazio) definido por $V_{\lambda}=\{x \in X:(\lambda, x) \in V\}$.

Observação 3.2.8. Na proposição acima, foi usado o termo invariância homotópica estendida porque a invariância homotópica clássica é usualmente apresentada para funções definidas em produtos $[0,1] \times \bar{W} \subset[0,1] \times X$ onde $W$ é aberto e limitado em $X$. 
Podemos finalmente enunciar a extensão que obtemos do Teorema 3.2.1.

Teorema 3.2.9. Se a funcão $f$ for contínua e se as condições do Lema 3.2.5 forem satisfeitas, então o problema (3.1) possuirá uma solução.

Demonstração. Seja $M$ o operador dado por (3.6). Usando a Proposição 3.2.7, podemos dizer que o grau de Leray-Schauder $\operatorname{deg}_{L S}\left(I-M(\lambda,),. V_{\lambda}, 0\right)$ está bem definido. Além disso, é constante para $\lambda \in[0,1]$. Por conseguinte,

$$
\operatorname{deg}_{L S}\left(I-M(0, .), V_{0}, 0\right)=\operatorname{deg}_{L S}\left(I-M(1, .), V_{1}, 0\right),
$$

onde $\operatorname{deg}_{L S}\left(I-M(0,),. V_{0}, 0\right)=\operatorname{deg}_{L S}\left(I, B_{\rho}(0), 0\right)=1$. Logo, pela propriedade de existência do grau (Proposição 2.4.8), existe $u \in V_{1}$ tal que $M(1, u)=u$, o que significa que $u$ é solução de (3.1).

Observação 3.2.10. É facil observar que o Teorema 3.2.1 é um caso particular do Teorema 3.2.9.

O corolário seguinte é uma simples consequência do Teorema 3.2.9.

Corolário 3.2.11. Sejam $\varphi$ um homeomorfismo crescente, $h \in C\left([0, T], \mathbb{R}^{+}\right)$e $f$ tais que

$$
\|h\|_{L^{1}}<a / 2,|f(t, x, y)| \leq f(t, x, y) x+h(t)
$$

para todo $x, y \in \mathbb{R}$ e todo $t \in[0, T]$. Então o problema (3.1) possuirá uma solução.

Demonstração. Como $\varphi$ é um homeomorfismo crescente, então

$$
\varphi(y) y \geq 0
$$

para todo $y \in \mathbb{R}$. Usando o Lema 3.2.5 com $n(x)=x$ para todo $x \in \mathbb{R}$ e o Teorema 3.2.9, provamos o resultado.

Concluímos a seção mostrando um exemplo de um problema cuja solução é obtida aplicando o Teorema 3.2.9 e que não pode ser abordado usando o Teorema 3.2.1 de Bereanu e Mawhin porque a função considerada $f$ se torna não limitada.

Exemplo 3.2.12. Sejam $\varphi: \mathbb{R} \longrightarrow(-1,1) \operatorname{com} \varphi(y)=\frac{y}{\sqrt{1+y^{2}}}, f(t, x, y)=x-2, h(t)=4$. Então usando o Corolário 3.2.11 o probema

$$
\left(\frac{u^{\prime}}{\sqrt{1+u^{\prime 2}}}\right)^{\prime}=u-2, u(0)=u(T)=0,
$$

tem ao menos uma solução se $T<1 / 8$.

\subsection{Problemas com homeomorfismos singulares em $\mathbb{R}$}

Nesta seção, estudamos a existência de pelo menos uma solução do seguinte problema que não conseguimos encontrar na literatura:

$$
\left\{\begin{array}{l}
\left(\varphi\left(u^{\prime}\right)\right)^{\prime}=f\left(t, u, u^{\prime}\right) \\
u(T)=u(0)=u^{\prime}(T),
\end{array}\right.
$$

onde $\varphi:(-a, a) \longrightarrow \mathbb{R}$ é um homeomorfismo tal que $\varphi(0)=0$ e $f:[0, T] \times \mathbb{R} \times \mathbb{R} \longrightarrow \mathbb{R}$ é uma função contínua.

Dizemos que uma função $u:[0, T] \longrightarrow \mathbb{R}$ de classe $C^{1}$ é solução do problema $(3.8)$ se $u^{\prime}(t) \in$ $(-a, a)$ para todo $t$ em $[0, T], u$ satisfaz as condições de fronteira e a função $\varphi\left(u^{\prime}\right)$ é de classe $C^{1} \mathrm{e}$ verifica $\left(\varphi\left(u^{\prime}(t)\right)\right)^{\prime}=f\left(t, u(t), u^{\prime}(t)\right)$ para todo $t$ em $[0, T]$.

O método utilizado aqui para obter uma solução de (3.8) consiste em reduzir o mesmo a uma aplicacão do Teorema do ponto fixo de Schauder (Teorema 2.4.10). Vamos aqui apresentar esta abordagem. 
Usando um argumento similar ao introduzido no Lema 3.2.2 para $h \in C$ e considerando a função $\varphi^{-1}$ com abuso de notação como o operador $\varphi^{-1}: C \longrightarrow B_{a}(0) \subset C$ definido por $\varphi^{-1}(v)(t)=$ $\varphi^{-1}(v(t))$, obtemos o seguinte teorema que estabelece uma relação direta do problema (3.8) com um operador definido em $C^{1}$.

Teorema 3.3.1. A função $u \in C^{1}$ é uma solução de (3.8) se, e somente se, u é um ponto fixo do operador $M$ definido sobre $C^{1}$ por

$$
M(u)=\varphi^{-1}\left(-Q_{\varphi}\left(K\left(N_{f}(u)\right)\right)\right)+H\left(\varphi^{-1}\left[K\left(N_{f}(u)\right)-Q_{\varphi}\left(K\left(N_{f}(u)\right)\right)\right]\right) .
$$

Demonstração. Se $u \in C^{1}$ é uma solução de (3.8), então $u$ satisfaz a equação (3.8), ou seja, para todo $t \in[0, T]$,

$$
\left(\varphi\left(u^{\prime}(t)\right)\right)^{\prime}=N_{f}(u)(t)=f\left(t, u(t), u^{\prime}(t)\right), u(0)=u(T), u(0)=u^{\prime}(T) .
$$

Aplicando $K$ e usando o fato de que $u(0)=u^{\prime}(T)$, segue que

$$
\varphi\left(u^{\prime}(t)\right)=\varphi(u(0))+K\left(N_{f}(u)\right)(t), u(0)=u(T) .
$$

Compondo com a função $\varphi^{-1}$, temos

$$
u^{\prime}(t)=\varphi^{-1}\left[K\left(N_{f}(u)\right)(t)+c\right], u(0)=u(T),
$$

onde $c=\varphi(u(0))$. Integrando de 0 a $t \in[0, T]$, deduzimos que, se $u$ é solução do problema (3.8), então

$$
u(t)=u(0)+H\left(\varphi^{-1}\left[K\left(N_{f}(u)\right)+c\right]\right)(t) .
$$

Como $u(0)=u(T)$, então

$$
\int_{0}^{T} \varphi^{-1}\left[K\left(N_{f}(u)\right)(t)+c\right] d t=0 .
$$

Assim, usando um argumento análogo ao Lema 3.2.2, temos que $c=-Q_{\varphi}\left(K\left(N_{f}(u)\right)\right)$. Portanto, $u$ satisfaz a equação

$$
u=\varphi^{-1}\left(-Q_{\varphi}\left(K\left(N_{f}(u)\right)\right)\right)+H\left(\varphi^{-1}\left[K\left(N_{f}(u)\right)-Q_{\varphi}\left(K\left(N_{f}(u)\right)\right)\right]\right)=M(u) .
$$

Reciprocamente, se $u \in C^{1}$ é um ponto fixo de $M$, então para todo $t \in[0, T]$,

$$
u(t)=\varphi^{-1}\left(-Q_{\varphi}\left(K\left(N_{f}(u)\right)\right)\right)+H\left(\varphi^{-1}\left[K\left(N_{f}(u)\right)-Q_{\varphi}\left(K\left(N_{f}(u)\right)\right)\right]\right)(t) .
$$

Como $\int_{0}^{T} \varphi^{-1}\left[K\left(N_{f}(u)\right)(t)-Q_{\varphi}\left(K\left(N_{f}(u)\right)\right)\right] d t=0$, então

$$
u(0)=u(T) .
$$

Derivando ambos os lados de (3.9), obtemos

$$
u^{\prime}(t)=\varphi^{-1}\left[K\left(N_{f}(u)\right)-Q_{\varphi}\left(K\left(N_{f}(u)\right)\right)\right](t) .
$$

Em particular, lembrando a definição de $K$,

$$
u^{\prime}(T)=\varphi^{-1}\left(-Q_{\varphi}\left(K\left(N_{f}(u)\right)\right)\right)=u(0) .
$$

Aplicando a função $\varphi$, segue que

$$
\varphi\left(u^{\prime}(t)\right)=K\left(N_{f}(u)\right)(t)-Q_{\varphi}\left(K\left(N_{f}(u)\right)\right), \quad u(0)=u(T), u(0)=u^{\prime}(T) .
$$

Agora, derive novamente e conclua que

$$
\left(\varphi\left(u^{\prime}(t)\right)\right)^{\prime}=N_{f}(u)(t), \quad u(0)=u(T), \quad u(0)=u^{\prime}(T)
$$

para todo $t \in[0, T]$. Portanto, $u$ é solução de (3.8). 
Conseguimos provar o seguinte lema por um argumento clássico usando o Teorema de ArzelàAscoli (Teorema 2.3.5). Este resultado de completa continuidade do operador é analogo ao da seção anterior, onde os operadores $M$ considerados aqui e na seção anterior não são iguais e, por conseguinte, damos a demonstração para maior clareza para o leitor.

Lema 3.3.2. O operador $M: C^{1} \longrightarrow C^{1}$ é completamente contínuo.

Demonstração. Seja $\Lambda$ um conjunto limitado de $C^{1}$. Então, existe $\rho>0$ tal que

$$
\|u\|_{1} \leq \rho
$$

para todo $u \in \Lambda$. Queremos mostrar que $\overline{M(\Lambda)} \subset C^{1}$ é um conjunto compacto. Para tanto, tome $\left(v_{n}\right)$ uma sequência em $M(\Lambda)$ e $\left(u_{n}\right)$ uma sequência em $\Lambda$ tal que $v_{n}=M\left(u_{n}\right)$. Da fórmula (3.10), segue que existe $L>0$ tal que

$$
\left\|N_{f}\left(u_{n}\right)\right\|_{\infty} \leq L
$$

para todo $n \in \mathbb{N}$. Daí se segue que

$$
\left\|K\left(N_{f}\left(u_{n}\right)\right)-Q_{\varphi}\left(K\left(N_{f}\left(u_{n}\right)\right)\right)\right\|_{\infty} \leq 2 L T .
$$

Portanto, a sequência $\left(K\left(N_{f}\left(u_{n}\right)\right)-Q_{\varphi}\left(K\left(N_{f}\left(u_{n}\right)\right)\right)\right)$ é limitada em $C$. Além disso, para todo $n \in \mathbb{N}$ e para $t, t_{1} \in[0, T]$, segue que

$$
\begin{aligned}
& \left|K\left(N_{f}\left(u_{n}\right)\right)(t)-Q_{\varphi}\left(K\left(N_{f}\left(u_{n}\right)\right)\right)-K\left(N_{f}\left(u_{n}\right)\right)\left(t_{1}\right)+Q_{\varphi}\left(K\left(N_{f}\left(u_{n}\right)\right)\right)\right| \\
& \leq\left|-\int_{t}^{T} f\left(s, u_{n}(s), u_{n}^{\prime}(s)\right) d s+\int_{t_{1}}^{T} f\left(s, u_{n}(s), u_{n}^{\prime}(s)\right) d s\right| \\
& \leq\left|\int_{t_{1}}^{t} f\left(s, u_{n}(s), u_{n}^{\prime}(s)\right) d s\right| \\
& \leq L\left|t-t_{1}\right|
\end{aligned}
$$

o que implica que a sequência $\left(K\left(N_{f}\left(u_{n}\right)\right)-Q_{\varphi}\left(K\left(N_{f}\left(u_{n}\right)\right)\right)\right)$ é equicontínua. Assim, usando o Teorema de Arzelà-Ascoli (Teorema 2.3.5), $\left(K\left(N_{f}\left(u_{n}\right)\right)-Q_{\varphi}\left(K\left(N_{f}\left(u_{n}\right)\right)\right)\right)$ possui uma subsequência convergente em $C$, que vamos chamar de

$$
\left(K\left(N_{f}\left(u_{n_{j}}\right)\right)-Q_{\varphi}\left(K\left(N_{f}\left(u_{n_{j}}\right)\right)\right)\right) .
$$

Sabendo que o operador $\varphi^{-1}: C \longrightarrow B_{a}(0) \subset C$ é contínuo e que

$$
M\left(u_{n_{j}}\right)^{\prime}=\varphi^{-1}\left[K\left(N_{f}\left(u_{n_{j}}\right)\right)-Q_{\varphi}\left(K\left(N_{f}\left(u_{n_{j}}\right)\right)\right)\right],
$$

segue que a sequência $\left(M\left(u_{n_{j}}\right)^{\prime}\right)$ é convergente em $C$. Então, passando a uma subsequência, se necessário, temos que a sequência $\left(v_{n_{j}}\right)=\left(M\left(u_{n_{j}}\right)\right)$ é convergente em $C^{1}$. Para finalizar a prova, suponha $\left(v_{n}\right) \subseteq \overline{M(\Lambda)}$. Seja $\left(z_{n}\right) \subseteq M(\Lambda)$ tal que

$$
\lim _{n \rightarrow \infty}\left\|z_{n}-v_{n}\right\|_{1}=0
$$

Seja também $\left(z_{n_{j}}\right)$ uma subsequência de $\left(z_{n}\right)$ que converge para $z$. Segue que $z \in \overline{M(\Lambda)}$ e $\left(v_{n_{j}}\right)$ converge para $z$. A prova está concluída.

A seguir temos o resultado principal desta seção que mostra a existência de pelo menos uma solução de (3.8).

Teorema 3.3.3. Para qualquer função contínua $f:[0, T] \times \mathbb{R} \times \mathbb{R} \longrightarrow \mathbb{R}$, o problema (3.8) tem ao menos uma solução.

Demonstração. Seja $u \in C^{1}$. Então,

$$
M(u)=\varphi^{-1}\left(-Q_{\varphi}\left(K\left(N_{f}(u)\right)\right)\right)+H\left(\varphi^{-1}\left[K\left(N_{f}(u)\right)-Q_{\varphi}\left(K\left(N_{f}(u)\right)\right)\right]\right),
$$


onde

$$
\begin{aligned}
& M(u)(0)=\varphi^{-1}\left(-Q_{\varphi}\left(K\left(N_{f}(u)\right)\right)\right)=M(u)(T), \\
& M(u)^{\prime}(T)=\varphi^{-1}\left(-Q_{\varphi}\left(K\left(N_{f}(u)\right)\right)\right)=M(u)(0) .
\end{aligned}
$$

Além disso,

$$
\left\|M(u)^{\prime}\right\|_{\infty}=\left\|\varphi^{-1}\left[K\left(N_{f}(u)\right)-Q_{\varphi}\left(K\left(N_{f}(u)\right)\right)\right]\right\|_{\infty}<a
$$

e

$$
\|M(u)\|_{\infty}<a+a T .
$$

Portanto,

$$
\|M(u)\|_{1}=\|M(u)\|_{\infty}+\left\|M(u)^{\prime}\right\|_{\infty}<a+a T+a=2 a+a T .
$$

Assim, o operador $M$ é uma aplicação de $C^{1}$ em $\overline{B_{2 a+a T}(0)}$ de $C^{1}$. Logo, pelo Teorema do ponto fixo de Schauder (Teorema (2.4.10)), $M$ tem um ponto fixo, que é solução de (3.8).

\subsubsection{Problemas do tipo misto com homeomorfismos singulares em $\mathbb{R}$}

Nesta subseção, operamos uma variação a respeito do problema anterior em que concerne as condições de contorno. Precisamente estamos interessados em problemas de valor de contorno do tipo

$$
\left\{\begin{array}{l}
\left(\varphi\left(u^{\prime}\right)\right)^{\prime}=f\left(t, u, u^{\prime}\right) \\
u(T)=u^{\prime}(0)=u^{\prime}(T),
\end{array}\right.
$$

onde $\varphi:(-a, a) \rightarrow \mathbb{R}$ é como na seção anterior um homeomorfismo tal que $\varphi(0)=0$ e $f$ : $[0, T] \times \mathbb{R} \times \mathbb{R} \longrightarrow \mathbb{R}$ uma função contínua.

A estratégia para mostrar a existência de ao menos uma solução de (3.11) é análoga à da seção anterior. Provamos que a existência de uma solução é equivalente a existência de um ponto fixo de um oportuno operador (o $M_{1}$ que vamos definir aqui junto). De fato, nesta seção o resultado de ponto fixo é provado usando o grau de Leray-Schauder, diversamente da seção anterior, onde foi usado o Teorema do ponto fixo de Schauder (Teorema (2.4.10)).

Vamos considerar o operador

$$
\begin{gathered}
M_{1}: C^{1} \rightarrow C^{1}, \\
u \mapsto S(u)+Q\left(N_{f}(u)\right)+K\left(\varphi^{-1}\left[H\left(N_{f}(u)-Q\left(N_{f}(u)\right)\right)+\varphi(S(u))\right]\right) .
\end{gathered}
$$

Quando $\varphi: \mathbb{R} \rightarrow \mathbb{R}$, tal operador foi considerado em [33].

Lema 3.3.4. Uma aplicação $u \in C^{1}$ é uma solução de (3.11) se, e somente se, u é um ponto fixo do operador $M_{1}$.

Demonstração. Seja $u \in C^{1}$. Temos as seguintes equivalências:

$$
\begin{aligned}
& \left(\varphi\left(u^{\prime}\right)\right)^{\prime}=N_{f}(u), u^{\prime}(T)=u^{\prime}(0), u^{\prime}(0)=u(T) \\
\Leftrightarrow & \left(\varphi\left(u^{\prime}\right)\right)^{\prime}=N_{f}(u)-Q\left(N_{f}(u)\right), \\
& Q\left(N_{f}(u)\right)=0, u^{\prime}(0)=u(T) \\
\Leftrightarrow & \varphi\left(u^{\prime}\right)=H\left(N_{f}(u)-Q\left(N_{f}(u)\right)\right)+\varphi\left(u^{\prime}(0)\right), \\
& Q\left(N_{f}(u)\right)=0, u^{\prime}(0)=u(T) \\
\Leftrightarrow & u^{\prime}=\varphi^{-1}\left[H\left(N_{f}(u)-Q\left(N_{f}(u)\right)+\varphi\left(u^{\prime}(0)\right)\right],\right. \\
& Q\left(N_{f}(u)\right)=0, u^{\prime}(0)=u(T) \\
\Leftrightarrow & u=u(T)+K\left(\varphi^{-1}\left[H\left(N_{f}(u)-Q\left(N_{f}(u)\right)\right)+\varphi\left(u^{\prime}(0)\right)\right]\right),
\end{aligned}
$$




$$
\begin{aligned}
& Q\left(N_{f}(u)\right)=0, u^{\prime}(0)=u(T) \\
\Leftrightarrow & u=u(T)+Q\left(N_{f}(u)\right)+K\left(\varphi^{-1}\left[H\left(N_{f}(u)-Q\left(N_{f}(u)\right)\right)+\varphi(u(T))\right]\right) \\
\Leftrightarrow & u=S(u)+Q\left(N_{f}(u)\right)+K\left(\varphi^{-1}\left[H\left(N_{f}(u)-Q\left(N_{f}(u)\right)\right)+\varphi(S(u))\right]\right) .
\end{aligned}
$$

Observação 3.3.5. Observe que $u^{\prime}(T)=u^{\prime}(0)$ se e somente se $Q\left(N_{f}(u)\right)=0$.

De modo semelhante ao Lema 3.3.2, temos o seguinte resultado do qual omitimos a demonstração.

Lema 3.3.6. O operador $M_{1}: C^{1} \rightarrow C^{1}$ é completamente contínuo.

A fim de aplicar o grau de Leray-Schauder para o operador ponto fixo $M_{1}$, introduzimos, para $\lambda \in[0,1]$, a família de problemas de valor de contorno

$$
\left\{\begin{array}{l}
\left(\varphi\left(u^{\prime}\right)\right)^{\prime}=\lambda N_{f}(u)+(1-\lambda) Q\left(N_{f}(u)\right) \\
u(T)=u^{\prime}(0)=u^{\prime}(T) .
\end{array}\right.
$$

Note-se que (3.12) coincide com (3.11) para $\lambda=1$. Assim, para cada $\lambda \in[0,1]$, o operador não linear associado ao problema (3.12) pelo Lema 3.3.4 é o operador $M(\lambda, \cdot)$, onde $M$ é definido sobre $[0,1] \times C^{1}\left(\right.$ com valores em $\left.C^{1}\right)$ por

$$
M(\lambda, u)=S(u)+Q\left(N_{f}(u)\right)+K\left(\varphi^{-1}\left[\lambda H\left(N_{f}(u)-Q\left(N_{f}(u)\right)\right)+\varphi(S(u))\right]\right) .
$$

Usando o Teorema de Arzelà-Ascoli, não é difícil ver que $M$ é completamente contínuo. Além disso, pelo raciocínio do Lema 3.3.4, vemos que o sistema (3.12) é equivalente ao problema

$$
u=M(\lambda, u) .
$$

O seguinte lema dá limitações a priori para os possíveis pontos fixos das aplicações $M(\lambda, \cdot)$.

Lema 3.3.7. Seja $f:[0, T] \times \mathbb{R} \times \mathbb{R} \rightarrow \mathbb{R}$ contínua. Se $(\lambda, u) \in[0,1] \times C^{1}$ é tal que $u=M(\lambda, u)$, então

$$
\|u\|_{1} \leq a(2+T) .
$$

Demonstração. Seja $[0, T] \times C^{1}$ tal que $u=M(\lambda, u)$. Então

$$
u=M(\lambda, u)=S(u)+Q\left(N_{f}(u)\right)+K\left(\varphi^{-1}\left[\lambda H\left(N_{f}(u)-Q\left(N_{f}(u)\right)\right)+\varphi(S(u))\right]\right) .
$$

Diferenciando, obtemos

$$
u^{\prime}=[M(\lambda, u)]^{\prime}=\varphi^{-1}\left[\lambda H\left(N_{f}(u)-Q\left(N_{f}(u)\right)\right)+\varphi(S(u))\right],
$$

de modo que

$$
\left\|u^{\prime}\right\|_{\infty} \leq a
$$

Como $u \in C^{1}$ é tal que $u(T)=u^{\prime}(0)$, temos que

$$
|u(t)| \leq|u(T)|+\int_{0}^{T}\left|u^{\prime}(s)\right| d s \leq a+a T \quad(t \in[0, T]),
$$

e portanto $\|u\|_{1}=\|u\|_{\infty}+\left\|u^{\prime}\right\|_{\infty} \leq a+a T+a=a(2+T)$.

Podemos agora fornecer um teorema de existência para (3.11). Lembramos os símbolos $\operatorname{deg}_{B}$ e $d_{e} g_{L S}$ para denotar o grau de Brouwer e o grau de Leray-Schauder, respectivamente, e definimos a aplicação contínua $G: \mathbb{R}^{2} \rightarrow \mathbb{R}^{2}$ por

$$
G: \mathbb{R}^{2} \rightarrow \mathbb{R}^{2},(x, y) \mapsto\left(x T+y T^{2}-y T-\frac{1}{T} \int_{0}^{T} f(t, x+y t, y) d t, y-x-y T\right) .
$$

Teorema 3.3.8. Seja $f:[0, T] \times \mathbb{R} \times \mathbb{R} \rightarrow \mathbb{R}$ uma função continua. Então para todo $\rho>a(2+T)$ 


$$
\operatorname{deg}_{L S}\left(I-M(1, \cdot), B_{\rho}(0), 0\right)=\operatorname{deg}_{B}\left(G, B_{\rho}(0) \cap \mathbb{R}^{2}, 0\right) .
$$

Se, além disso,

$$
\operatorname{deg}_{B}\left(G, B_{\rho}(0) \cap \mathbb{R}^{2}, 0\right) \neq 0,
$$

o problema (3.11) tem pelo menos uma solução.

Demonstração. Seja $M$ o operador dado por (3.13) e seja $\rho>a(2+T)$. Usando o Lema 3.3.7, temos que, para cada $\lambda \in[0, T]$, o grau de Leray-Schauder $\operatorname{deg}_{L S}\left(I-M(\lambda, \cdot), B_{\rho}(0), 0\right)$ está bem definido, e pela propriedade de invariância homotópica, temos

$$
\operatorname{deg}_{L S}\left(I-M(0, \cdot), B_{\rho}(0), 0\right)=\operatorname{deg}_{L S}\left(I-M(1, \cdot), B_{\rho}(0), 0\right) .
$$

Por outro lado, temos que

$$
\operatorname{deg}_{L S}\left(I-M(0, \cdot), B_{\rho}(0), 0\right)=\operatorname{deg}_{L S}\left(I-\left(S+Q N_{f}+K S\right), B_{\rho}(0), 0\right) .
$$

Mas a imagem da aplicação

$$
u \mapsto S(u)+Q\left(N_{f}(u)\right)+K(S(u))
$$

está contida no subespaço das funções afins, isomorfo a $\mathbb{R}^{2}$. Usando a propriedade de invariância homotópica e propriedades de redução do grau de Leray-Schauder [15], obtemos

$$
\begin{aligned}
& \operatorname{deg}_{L S}\left(I-\left(S+Q N_{f}+K S\right), B_{\rho}(0), 0\right) \\
& =\operatorname{deg}_{B}\left(I-\left(S+Q N_{f}+K S\right) \mid \overline{B_{\rho}(0) \cap \mathbb{R}^{2}}, B_{\rho}(0) \cap \mathbb{R}^{2}, 0\right) \\
& =\operatorname{deg}_{B}\left(G, B_{\rho}(0) \cap \mathbb{R}^{2}, 0\right) \neq 0 .
\end{aligned}
$$

Então, $\operatorname{deg}_{L S}\left(I-M(1, \cdot), B_{\rho}(0), 0\right) \neq 0$. Portanto, existe $u \in B_{\rho}(0)$ tal que $M_{1}(u)=u$, que é uma solução de (3.11).

Vamos dar agora uma aplicação do Teorema 3.3.8.

Exemplo 3.3.9. Consideramos o problema

$$
\left\{\begin{array}{l}
\left(\varphi\left(u^{\prime}\right)\right)^{\prime}=e^{u^{\prime}}+e \\
u(T)=u^{\prime}(0)=u^{\prime}(T)
\end{array}\right.
$$

onde $\varphi(s)=\frac{s}{\sqrt{1-s^{2}}}$.

$N a \tilde{o}$ é difícil de verificar que $\varphi:(-1,1) \rightarrow \mathbb{R}$ é um homeomorfismo e que $f(t, x, y)=e^{y}+e$ é uma função contínua. Se escolhermos $\rho>2+T$, temos que a equação

$$
\begin{aligned}
G(x, y) & =\left(x T+y T^{2}-y T-\frac{1}{T} \int_{0}^{T} f(t, x+y t, y) d t, y-x-y T\right)=(0,0) \\
& =\left(x T+y T^{2}-y T-\frac{1}{T} \int_{0}^{T}\left(e^{y}-e\right) d t, y-x-y T\right)=(0,0) \\
& =\left(x T+y T^{2}-y T-e^{y}+e, y-x-y T\right)=(0,0)
\end{aligned}
$$

não tem solução em $\partial B_{\rho}(0) \cap \mathbb{R}^{2}$. Então temos que o grau de Brouwer deg $g_{B}\left(G, B_{\rho}(0) \cap \mathbb{R}^{2},(0,0)\right)$ está bem definido e, pelas propriedades do grau, temos que

$$
\operatorname{deg}_{B}\left(G, B_{\rho}(0) \cap \mathbb{R}^{2},(0,0)\right)=\sum_{(x, y) \in G^{-1}(0,0)} \operatorname{sgn}\left(\operatorname{det}\left(G^{\prime}(x, y)\right)\right)=\operatorname{sgn}(-e)=-1,
$$

onde $(0,0)$ é um valor regular de $G$. Assim, usando o Teorema 3.3.8, obtemos que o problema (3.14) tem ao menos uma solução. 
Observação 3.3.10. Usando a família de problemas de valor de contorno

$$
\left\{\begin{array}{l}
\left(\varphi\left(u^{\prime}\right)\right)^{\prime}=\lambda N_{f}(u)+(1-\lambda) Q\left(N_{f}(u)\right) \\
u(0)=u^{\prime}(0)=u^{\prime}(T)
\end{array}\right.
$$

o que dá a homotopia completamente contínua $\widetilde{M}$ definida em $[0,1] \times C^{1}$ por

$$
\widetilde{M}(\lambda, u)=P(u)+Q\left(N_{f}(u)\right)+H\left(\varphi^{-1}\left[\lambda H\left(N_{f}(u)-Q\left(N_{f}(u)\right)\right)+\varphi(P(u))\right]\right),
$$

não é difícil ver que (3.15) tem uma solução para $\lambda=1$.

\subsection{Problemas com homeomorfismos limitados em $\mathbb{R}$}

Nesta seção, estudamos a existência de soluções para problemas de valor de contorno da forma

$$
\left\{\begin{array}{l}
\left(\varphi\left(u^{\prime}\right)\right)^{\prime}=f\left(t, u, u^{\prime}\right) \\
u(0)=u^{\prime}(0)=u^{\prime}(T),
\end{array}\right.
$$

onde $\varphi: \mathbb{R} \rightarrow(-a, a)$ é um homeomorfismo, $\varphi(0)=0$ e $f:[0, T] \times \mathbb{R} \times \mathbb{R} \rightarrow \mathbb{R}$ é uma função contínua. Quando as condições de contorno são periódicas ou Neumann, em (3.16) Bereanu e Mawhin em [8] estabelecem resultados de existência.

Como na subseção acima, queremos aplicar o grau de Leray-Schauder para abordar o problema (3.16). Com esta finalidade, introduzimos para $\lambda \in[0,1]$, a família de problemas de valor de contorno

$$
\left\{\begin{array}{l}
\left(\varphi\left(u^{\prime}\right)\right)^{\prime}=\lambda N_{f}(u)+(1-\lambda) Q\left(N_{f}(u)\right) \\
u(0)=u^{\prime}(0)=u^{\prime}(T) .
\end{array}\right.
$$

Observe que (3.17) coincide, para $\lambda=1$, com (3.16). Agora, introduzimos o conjunto

$$
\Omega=\left\{(\lambda, u) \in[0,1] \times C^{1}:\left\|\lambda H\left(N_{f}(u)-Q\left(N_{f}(u)\right)\right)+\varphi(P(u))\right\|_{\infty}<a\right\},
$$

que é aberto em $[0,1] \times C^{1}$, e não vazio porque $\{0\} \times C^{1} \subset \Omega$. Além disso, introduzimos o operador $M: \Omega \rightarrow C^{1}$ definido por

$$
M(\lambda, u)=P(u)+Q\left(N_{f}(u)\right)+H\left(\varphi^{-1}\left[\lambda H\left(N_{f}(u)-Q\left(N_{f}(u)\right)\right)+\varphi(P(u))\right]\right) .
$$

Aqui, por um abuso de notação, $\varphi^{-1}$ é entendida como uma função $\varphi^{-1}: B_{a}(0) \subset C \rightarrow C$ dada por $\varphi^{-1}(v)(t)=\varphi^{-1}(v(t))$. O símbolo $B_{a}(0)$ denota a bola aberta em $C$ de centro 0 e raio $a$. É claro que $\varphi^{-1}$ é contínua.

O seguinte lema desempenha um papel fundamental para estudar as soluções do problema (3.17).

Lema 3.4.1. O operador $M: \Omega \rightarrow C^{1}$ está bem definido e é contínuo. Além disso, se $(\lambda, u) \in \Omega$ é tal que $M(\lambda, u)=u$, então u é uma solução de (3.17).

Demonstração. Seja $(\lambda, u) \in \Omega$. Vamos mostrar que de fato $M(\lambda, u) \in C^{1}$. A continuidade de $M(\lambda, u)$ segue do fato de que é uma composição de funções contínuas. Além disso, temos que

$$
(M(\lambda, u))^{\prime}=\varphi^{-1}\left[\lambda H\left(N_{f}(u)-Q\left(N_{f}(u)\right)\right)+\varphi(P(u))\right],
$$

que é uma composição de funções contínuas. Desta forma, concluímos que $M(\lambda, u) \in C^{1}$. A continuidade de $M$ segue da continuidade dos operadores que compõem $M$.

Agora, suponha que $(\lambda, u) \in \Omega$ seja tal que $M(\lambda, u)=u$. Resulta de (3.18) que

$$
u(t)=u(0)+Q\left(N_{f}(u)\right)(t)+H\left(\varphi^{-1}\left[\lambda H\left(N_{f}(u)-Q\left(N_{f}(u)\right)\right)+\varphi(P(u))\right]\right)(t)
$$


para todo $t \in[0, T]$. Então, tomando $t=0$, segue que

$$
Q\left(N_{f}(u)\right)=0 .
$$

Derivando (3.19), obtemos

$$
\begin{aligned}
u^{\prime}(t) & =\varphi^{-1}\left[\lambda H\left(N_{f}(u)-Q\left(N_{f}(u)\right)\right)+\varphi(P(u))\right](t) \\
& =\varphi^{-1}\left[\lambda H\left(N_{f}(u)-Q\left(N_{f}(u)\right)\right)(t)+\varphi(u(0))\right] .
\end{aligned}
$$

Em particular,

$$
u(0)=u^{\prime}(0)=u^{\prime}(T)
$$

Aplicando a função $\varphi$, derivando novamente, e usando (3.20), temos

$$
\left(\varphi\left(u^{\prime}(t)\right)\right)^{\prime}=\lambda N_{f}(u)+(1-\lambda) Q\left(N_{f}(u)\right)(t)
$$

pata todo $t \in[0, T]$. Assim, $u$ satisfaz (3.17). Isso completa a prova.

Observação 3.4.2. Note-se que, para $\lambda \in[0,1]$, se $u$ é uma solução de $(3.17)$, então $Q\left(N_{f}(u)\right)=0$.

O seguinte lema dá uma limitação a priori para os possíveis pontos fixos de $M$.

Lema 3.4.3. Suponha que $f$ satisfaça as seguintes condições.

1. Existem $M_{1}<M_{2}$ tais que para todo $u \in C^{1}$

$$
\begin{aligned}
& \int_{0}^{T} f\left(t, u(t), u^{\prime}(t)\right) d t \neq 0 \quad \text { se } \quad u_{m}^{\prime} \geq M_{2}, \\
& \int_{0}^{T} f\left(t, u(t), u^{\prime}(t)\right) d t \neq 0 \quad \text { se } \quad u_{M}^{\prime} \leq M_{1} .
\end{aligned}
$$

2. Existe $c \in C$ tal que

$$
f(t, x, y) \geq c(t) \quad e \quad L+2\left\|c^{-}\right\|_{L^{1}}<a
$$

para todo $(t, x, y) \in[0, T] \times \mathbb{R} \times \mathbb{R}$ e $L=\max \left\{\left|\varphi\left(M_{2}\right)\right|,\left|\varphi\left(M_{1}\right)\right|\right\}$. Acima, $c^{-}=\max \{-c, 0\}$.

Se $(\lambda, u) \in \Omega$ é tal que $u=M(\lambda, u)$, então

$$
\left\|\lambda H\left(N_{f}(u)-Q\left(N_{f}(u)\right)\right)+\varphi(P(u))\right\|_{\infty}<L+2\left\|c^{-}\right\|_{L^{1}} \quad \text { e }\|u\|_{1}<r(2+T),
$$

onde

$$
r=\max \left\{\left|\varphi^{-1}\left(L+2\left\|c^{-}\right\|_{L^{1}}\right)\right|,\left|\varphi^{-1}\left(-L-2\left\|c^{-}\right\|_{L^{1}}\right)\right|\right\} .
$$

Demonstração. Seja $(\lambda, u) \in \Omega$ tal que $u=M(\lambda, u)$. Usando o Lemma 3.2.3, u é uma solução de (3.17), então

$$
Q\left(N_{f}(u)\right)=0
$$

e, assim, $u$ resolve o problema

$$
\left(\varphi\left(u^{\prime}\right)\right)^{\prime}=\lambda N_{f}(u), \quad u(0)=u^{\prime}(0)=u^{\prime}(T) .
$$

Portanto,

$$
\varphi\left(u^{\prime}(t)\right)=\lambda H\left(N_{f}(u)-Q\left(N_{f}(u)\right)\right)(t)+\varphi(u(0)) \quad(t \in[0, T]) .
$$

Por outro lado usando a hipótese 1, existem

$$
u_{m}^{\prime}<M_{2} \text { e } u_{M}^{\prime}>M_{1} .
$$

Daqui resulta que existe $\omega \in[0, T]$ tal que $M_{1}<u^{\prime}(\omega)<M_{2}$ e

$$
\int_{\omega}^{t}\left(\varphi\left(u^{\prime}(s)\right)\right)^{\prime} d s=\lambda \int_{\omega}^{t} N_{f}(u)(s) d s,
$$


o que implica que

$$
\left|\varphi\left(u^{\prime}(t)\right)\right| \leq\left|\varphi\left(u^{\prime}(\omega)\right)\right|+\int_{0}^{T}\left|f\left(s, u(s), u^{\prime}(s)\right)\right| d s,
$$

onde

$$
\int_{0}^{T}\left|f\left(s, u(s), u^{\prime}(s)\right)\right| d s \leq \int_{0}^{T} f\left(s, u(s), u^{\prime}(s)\right) d s+2 \int_{0}^{T} c^{-}(s) d s
$$

Portanto,

$$
\left|\varphi\left(u^{\prime}(t)\right)\right|<L+2\left\|c^{-}\right\|_{L^{1}},
$$

onde $L=\max \left\{\left|\varphi\left(M_{2}\right)\right|,\left|\varphi\left(M_{1}\right)\right|\right\}$ e $t \in[0, T]$. Usando a igualdade (3.22), segue que

$$
\left\|\lambda H\left(N_{f}(u)-Q\left(N_{f}(u)\right)\right)+\varphi(P(u))\right\|_{\infty}<L+2\left\|c^{-}\right\|_{L^{1}}<a,
$$

que fornece

$$
\left\|u^{\prime}\right\|_{\infty}<r
$$

onde $r=\max \left\{\left|\varphi^{-1}\left(L+2\left\|c^{-}\right\|_{L^{1}}\right)\right|,\left|\varphi^{-1}\left(-L-2\left\|c^{-}\right\|_{L^{1}}\right)\right|\right\}$. Como $u \in C^{1}$ é tal que $u^{\prime}(0)=u(0)$ temos que

$$
|u(t)| \leq|u(0)|+\int_{0}^{T}\left|u^{\prime}(s)\right| d s<r+r T \quad(t \in[0, T]) .
$$

Assim, obtemos que $\|u\|_{1}=\|u\|_{\infty}+\left\|u^{\prime}\right\|_{\infty}<r+r T+r=r(2+T)$. Isso completa a prova.

Sejam agora $\rho, \kappa \in \mathbb{R}$ tais que $L+2\left\|c^{-}\right\|_{L^{1}}<\kappa<a, \rho>r(2+T)$ e consideramos o conjunto

$$
V=\left\{(\lambda, u) \in[0,1] \times C^{1}:\left\|\lambda H\left(N_{f}(u)-Q\left(N_{f}(u)\right)\right)+\varphi(P(u))\right\|_{\infty}<\kappa,\|u\|_{1}<\rho\right\} .
$$

Como o conjunto $\{0\} \times\left\{u \in C^{1}:\|u\|_{1}<\rho,\|\varphi(P(u))\|_{\infty}<\kappa\right\} \subset V$, então deduzimos que $V$ é não vazio. Além disso, é evidente que $V$ é aberto e limitado em $[0,1] \times C^{1}$ e $\bar{V} \subset \Omega$. Por outro lado, usando um argumento similar ao apresentado na prova do Lema 3.2.6, não é difícil ver que $M: \bar{V} \rightarrow C^{1}$ está bem definido e completamente contínuo. Além disso, usando o Lema 3.4.3, temos

$$
u \neq M(\lambda, u) \text { para todo }(\lambda, u) \in \partial V .
$$

Apresentamos finalmente um teorema de existência para (3.16). Definimos a aplicação $G: \mathbb{R}^{2} \rightarrow$ $\mathbb{R}^{2}$ por

$$
G: \mathbb{R}^{2} \rightarrow \mathbb{R}^{2},(x, y) \mapsto\left(-\frac{1}{T} \int_{0}^{T} f(t, x+y t, y) d t, y-x\right) .
$$

Teorema 3.4.4. Seja $f:[0, T] \times \mathbb{R} \times \mathbb{R} \rightarrow \mathbb{R}$ uma função contínua satisfazendo as condições (1) $e$ (2) do Lema 3.4.3. Então, para $\rho>r(2+T)$ e $\kappa \in \mathbb{R}$ com $L+2\left\|c^{-}\right\|_{L^{1}}<\kappa<$ a, temos

$$
\operatorname{deg}_{L S}\left(I-M(1, \cdot), V_{1}, 0\right)=\operatorname{deg}_{B}(G, \Delta, 0),
$$

onde $V_{1}$ é o conjunto aberto limitado definido por $V_{1}=\left\{u \in C^{1}:(1, u) \in V\right\}$ e $\Delta=B_{\rho}(0) \cap \mathbb{R}^{2} \cap$ $\left\{(x, y) \in \mathbb{R}^{2}:|\varphi(x)|<\kappa\right\}$. Se, além disso,

$$
\operatorname{deg}_{B}(G, \Delta, 0) \neq 0
$$

problema (3.16) tem pelo menos uma solução.

Demonstração. Fixamos $\rho>r(2+T)$ e $\kappa \in \mathbb{R} \operatorname{com} L+2\left\|c^{-}\right\|_{L^{1}}<\kappa<a$ e seja $M$ o operador dado por (3.18) definido sobre $\bar{V}$. Usando a Proposição 3.2.7, deduzimos que

$$
\operatorname{deg}_{L S}\left(I-M(0, .), V_{0}, 0\right)=\operatorname{deg}_{L S}\left(I-M(1, .), V_{1}, 0\right),
$$

Por outro lado, temos que

$$
\operatorname{deg}_{L S}\left(I-M(0, .), V_{0}, 0\right)=\operatorname{deg}_{L S}\left(I-\left(P+Q N_{f}+H P\right), V_{0}, 0\right) .
$$

Mas o conjunto imagem da aplicação

$$
u \mapsto P(u)+Q\left(N_{f}(u)\right)+H(P(u))
$$


está contido no subespaço das funções afins isomorfo a $\mathbb{R}^{2}$. Utilizando as propriedades de invariância homotópica e de redução do grau de Leray-Schauder [15], obtemos

$$
\begin{aligned}
& \operatorname{deg}_{L S}\left(I-\left(P+Q N_{f}+H P\right), V_{0}, 0\right) \\
& =\operatorname{deg}_{B}\left(I-\left.\left(P+Q N_{f}+H P\right)\right|_{\bar{\Delta}}, \Delta, 0\right) \\
& =\operatorname{deg}_{B}(G, \Delta, 0) \neq 0 .
\end{aligned}
$$

Então, $\operatorname{deg}_{L S}\left(I-M(1,),. V_{1}, 0\right) \neq 0$. Isto implica que, existe $u \in V_{1}$ tal que $M(1, u)=u$, que é uma solução para (3.16).

Observação 3.4.5. Usando a família de problemas de valor de contorno

$$
\left\{\begin{array}{l}
\left(\varphi\left(u^{\prime}\right)\right)^{\prime}=\lambda N_{f}(u)+(1-\lambda) Q\left(N_{f}(u)\right) \\
u(T)=u^{\prime}(0)=u^{\prime}(T)
\end{array}\right.
$$

o que dá a homotopia completamente contínua $\widetilde{M}$ definida por

$$
\widetilde{M}(\lambda, u)=S(u)+Q\left(N_{f}(u)\right)+K\left(\varphi^{-1}\left[\lambda H\left(N_{f}(u)-Q\left(N_{f}(u)\right)\right)+\varphi(S(u))\right]\right),
$$

e similares limitações a priori como no Lema 3.4.3, não é difícil ver que (3.23) tem uma solução para $\lambda=1$.

Vamos dar agora uma aplicação do Teorema 3.4.4.

Exemplo 3.4.6. Considere o problema de valor de contorno

$$
\left\{\begin{array}{l}
\left(\frac{u^{\prime}}{\sqrt{1+u^{\prime 2}}}\right)^{\prime}=e^{4 u^{\prime}}-e \\
u(0)=u^{\prime}(0)=u^{\prime}(T) .
\end{array}\right.
$$

Seja $u \in C^{1}, M_{1}=0$ e $M_{2}=\frac{1}{2}$. Se supusermos que $u_{m}^{\prime} \geq M_{2}$ ou $u_{M}^{\prime} \leq M_{1}$, então

$$
\int_{0}^{T}\left(e^{4 u^{\prime}(t)}-e\right) d t \geq\left(e^{2}-e\right) T>0, \quad \int_{0}^{T}\left(e^{4 u^{\prime}(t)}-e\right) d t \leq(1-e) T<0 .
$$

Seja $c(t)=-3$ para todo $t \in[0, T]$, e seja $L=\frac{1}{\sqrt{5}}$. Se $L+6 T<\kappa=0,9<1$ e $\rho>r(2+T)=$ $\frac{L+6 T}{\sqrt{1-(L+6 T)^{2}}}(2+T)$, então a equação

$$
\begin{aligned}
G(x, y) & =\left(-\frac{1}{T} \int_{0}^{T} f(t, x+y t, y) d t, y-x\right)=(0,0) \\
& =\left(-\frac{1}{T} \int_{0}^{T}\left(e^{4 y}-e\right) d t, y-x\right)=(0,0) \\
& =\left(-e^{4 y}+e, y-x\right)=(0,0)
\end{aligned}
$$

não tem solução em $\partial \Delta$, e portanto o grau de Brouwer deg $g_{B}(G, \Delta,(0,0))$ está bem definido. Assim, utilizando as propriedades do grau de Brouwer, temos

$$
\operatorname{deg}_{B}(G, \Delta,(0,0))=\sum_{(x, y) \in G^{-1}(0,0)} \operatorname{sgn}\left(\operatorname{det}\left(G^{\prime}(x, y)\right)\right) \neq 0,
$$

onde $(0,0)$ é um valor regular de $G$. Por conseguinte, o problema (3.24) tem pelo menos uma solução. 


\subsubsection{Problemas do tipo misto com homeomorfismos limitados em $\mathbb{R}$}

Nesta subseção, vamos estudar a existência de soluções para problemas do tipo

$$
\left\{\begin{array}{l}
\left(\varphi\left(u^{\prime}\right)\right)^{\prime}=f\left(t, u, u^{\prime}\right) \\
u(T)=u(0)=u^{\prime}(T),
\end{array}\right.
$$

onde $\varphi: \mathbb{R} \rightarrow(-a, a)$ é um homeomorfismo, $\varphi(0)=0$ e $f:[0, T] \times \mathbb{R} \times \mathbb{R} \rightarrow \mathbb{R}$ é uma função contínua tal que

$$
|f(t, x, y)| \leq c<\frac{a}{2 T} \quad \text { para todo }(t, x, y) \in[0, T] \times \mathbb{R} \times \mathbb{R} .
$$

A estratégia para procurar soluções é análoga à das seções anteriores: vamos reduzir (3.25) a um problema de ponto fixo. A aplicação do grau de Leray-Schauder irá nos dar a existência de soluções do problema (3.25).

Aplicamos o Lema 3.2.2, usando a função $Q_{\varphi}$ do lema, e a condição (3.26) introduzimos o operador $M_{1}: C^{1} \rightarrow C^{1}$ definido por

$$
M_{1}(u)=\varphi^{-1}\left(-Q_{\varphi}\left(K\left(N_{f}(u)\right)\right)\right)+H\left(\varphi^{-1}\left[K\left(N_{f}(u)\right)-Q_{\varphi}\left(K\left(N_{f}(u)\right)\right)\right]\right) .
$$

Os seguintes resultados foram apresentados em [33]. As provas são semelhantes às estabelecidas no Teorema 3.3.1 e Lema 3.3.2, respectivamente.

Lema 3.4.7. Se $u \in C^{1}$ é tal que $u=M_{1}(u)$, então u é uma solução de (3.25).

Lema 3.4.8. O operador $M_{1}: C^{1} \rightarrow C^{1}$ é completamente contínuo.

A fim de aplicar o grau de Leray-Schauder para o operador ponto fixo $M_{1}$, introduzimos, para $\lambda \in[0,1]$, a família de problemas de valor de contorno

$$
\left\{\begin{array}{l}
\left(\varphi\left(u^{\prime}\right)\right)^{\prime}=\lambda f\left(t, u, u^{\prime}\right) \\
u(T)=u(0)=u^{\prime}(T) .
\end{array}\right.
$$

Observe que (3.27) coincide com (3.25) para $\lambda=1$. Para cada $\lambda \in[0,1]$, podemos definir em $C^{1}$ o operador $M(\lambda, \cdot)$, onde $M$ é definido em $[0,1] \times C^{1}$ por

$$
M(\lambda, u)=\varphi^{-1}\left(-Q_{\varphi}\left(\lambda K\left(N_{f}(u)\right)\right)\right)+H\left(\varphi^{-1}\left[\lambda K\left(N_{f}(u)\right)-Q_{\varphi}\left(\lambda K\left(N_{f}(u)\right)\right)\right]\right) .
$$

Usando o Teorema de Arzelà-Ascoli não é difícil ver que $M$ é completamente contínuo.

Lema 3.4.9. Se $(\lambda, u) \in[0,1] \times C^{1}$ é tal que $u=M(\lambda, u)$, então u é uma solução de (3.27).

Demonstração. Seja $(\lambda, u) \in[0,1] \times C^{1}$ tal que $u=M(\lambda, u)$. Então

$$
u(t)=\varphi^{-1}\left(-Q_{\varphi}\left(\lambda K\left(N_{f}(u)\right)\right)\right)+H\left(\varphi^{-1}\left[\lambda K\left(N_{f}(u)\right)-Q_{\varphi}\left(\lambda K\left(N_{f}(u)\right)\right)\right]\right)(t)
$$

para todo $t \in[0, T]$. Como $\int_{0}^{T} \varphi^{-1}\left[\lambda K\left(N_{f}(u)\right)(t)-Q_{\varphi}\left(\lambda K\left(N_{f}(u)\right)\right)\right] d t=0$, por conseguinte, temos que $u(0)=u(T)$. Diferenciando (3.29), obtemos

$$
u^{\prime}(t)=\varphi^{-1}\left[\lambda K\left(N_{f}(u)\right)-Q_{\varphi}\left(\lambda K\left(N_{f}(u)\right)\right)\right](t) .
$$

Em particular,

$$
u^{\prime}(T)=\varphi^{-1}\left(0-Q_{\varphi}\left(\lambda K\left(N_{f}(u)\right)\right)\right)=\varphi^{-1}\left(-Q_{\varphi}\left(\lambda K\left(N_{f}(u)\right)\right)\right)=u(0) .
$$

Aplicando $\varphi$, e diferenciando novamente, segue que

$$
\left(\varphi\left(u^{\prime}(t)\right)\right)^{\prime}=\lambda N_{f}(u)(t), \quad u(0)=u(T), u(0)=u^{\prime}(T)
$$

para todo $t \in[0, T]$. Isso completa a prova. 
Observação 3.4.10. Note-se que o recíproco do Lema 3.4.9 é verdadeiro, porque podemos garantir que $\| \lambda K\left(N_{f}(u) \|_{\infty}<a / 2\right.$ para toda solução $u$ de (3.27).

Agora vamos mostrar a existência de pelo menos uma solução para o problema (3.8) por meio do grau de Leray-Schauder. Este resultado é inspirado em trabalhos de Bereanu e Mawhin [8].

Teorema 3.4.11. Seja $f:[0, T] \times \mathbb{R} \times \mathbb{R} \longrightarrow \mathbb{R}$ uma função contínua. Se $f$ satisfaz a condição (3.26), então o problema (3.25) tem ao menos uma solução.

Demonstração. Seja $(\lambda, u) \in[0,1] \times C^{1}$ tal que $u=M(\lambda, u)$. Então

$$
u(t)=\varphi^{-1}\left(-Q_{\varphi}\left(\lambda K\left(N_{f}(u)\right)\right)\right)+H\left(\varphi^{-1}\left[\lambda K\left(N_{f}(u)\right)-Q_{\varphi}\left(\lambda K\left(N_{f}(u)\right)\right)\right]\right)(t)
$$

para todo $t \in[0, T]$. Diferenciando, temos

$$
\begin{aligned}
u^{\prime}(t) & =\varphi^{-1}\left[\lambda K\left(N_{f}(u)\right)-Q_{\varphi}\left(\lambda K\left(N_{f}(u)\right)\right)\right](t) \\
& =\varphi^{-1}\left[\lambda K\left(N_{f}(u)\right)(t)-Q_{\varphi}\left(\lambda K\left(N_{f}(u)\right)\right)\right] .
\end{aligned}
$$

Apicando $\varphi$, segue que

$$
\varphi\left(u^{\prime}(t)\right)=\lambda K\left(N_{f}(u)\right)(t)-Q_{\varphi}\left(\lambda K\left(N_{f}(u)\right)\right) \quad(t \in[0, T]) .
$$

Usando o fato de que $f$ é limitada, deduzimos a desigualdade elementar

$$
\left\|\varphi\left(u^{\prime}\right)\right\|_{\infty} \leq 2 c T<a .
$$

Portanto,

$$
\left\|u^{\prime}\right\|_{\infty} \leq L
$$

onde $L=\max \left\{\left|\varphi^{-1}(2 c T)\right|,\left|\varphi^{-1}(-2 c T)\right|\right\}$. Como $u \in C^{1}$ é tal que $u(0)=u^{\prime}(T)$, temos que

$$
|u(t)| \leq|u(0)|+\int_{0}^{T}\left|u^{\prime}(s)\right| d s \leq L+L T \quad(t \in[0, T]),
$$

e portanto

$$
\|u\|_{1}=\|u\|_{\infty}+\left\|u^{\prime}\right\|_{\infty} \leq L+L T+L=L(2+T) .
$$

Seja $M$ o operador dado por (3.28), e seja $\rho>L(2+T)$. Usando a invariância homotópica do grau Leray-Schauder, temos

$$
\operatorname{deg}_{L S}\left(I-M(0, \cdot), B_{\rho}(0), 0\right)=\operatorname{deg}_{L S}\left(I-M(1, \cdot), B_{\rho}(0), 0\right) .
$$

Por outro lado, temos que

$$
\operatorname{deg}_{L S}\left(I-M(0, \cdot), B_{\rho}(0), 0\right)=\operatorname{deg}_{L S}\left(I, B_{\rho}(0), 0\right)=1 .
$$

Então, a partir da propriedade de existência do grau de Leray-Schauder [15], existe $u \in B_{\rho}(0)$ tal que $u=M(1, u)$, que é uma solução de (3.25).

Exemplo 3.4.12. Considere o problema de valor de contorno

$$
\left\{\begin{array}{l}
\left(\frac{u^{\prime}}{\sqrt{1+u^{\prime 2}}}\right)^{\prime}=\beta \cos u \\
u(0)=u(T)=u^{\prime}(T) .
\end{array}\right.
$$

Então, podemos escolher $\beta<\frac{1}{2 T}$ para aplicar Teorema 3.4.11 e assim o problema (3.30) tem uma solução. 


\subsection{Problemas do tipo misto com homeomorfismos clássicos em $\mathbb{R}$}

Nesta seção estamos interessados em obter uma solução para uma classe de problemas da forma

$$
\left\{\begin{array}{l}
\left(\varphi\left(u^{\prime}\right)\right)^{\prime}=f\left(t, u, u^{\prime}\right) \\
u(T)=u^{\prime}(0)=u^{\prime}(T),
\end{array}\right.
$$

onde $\varphi: \mathbb{R} \longrightarrow \mathbb{R}$ é um homeomorfismo tal que $\varphi(0)=0$ e $f:[0, T] \times \mathbb{R} \times \mathbb{R} \longrightarrow \mathbb{R}$ uma função contínua.

Entendemos por solução do problema (3.31) uma função $u:[0, T] \longrightarrow \mathbb{R}$ de classe $C^{1}$ satisfazendo as condições de fronteira e tal que a função $t \longmapsto \varphi\left(u^{\prime}(t)\right)$ seja de classe $C^{1}$ e satisfaça $\left(\varphi\left(u^{\prime}(t)\right)\right)^{\prime}=f\left(t, u(t), u^{\prime}(t)\right)$ para todo $t$ em $[0, T]$.

Procedemos analogamente às seções anteriores. O símbolo $\varphi^{-1}$ continua denotando com abuso de notação o operador $\varphi^{-1}: C \longrightarrow C$ definido por $\varphi^{-1}(v)(t)=\varphi^{-1}(v(t))$. Claramente $\varphi^{-1}$ é contínuo e envia conjuntos limitados em conjuntos limitados.

Usando o fato acima obtemos o seguinte resultado.

Lema 3.5.1. $u \in C^{1}$ é uma solução de (3.31) se e somente se u é um ponto fixo do operador $M_{1}$ definido sobre $C^{1}$ por

$$
M_{1}(u)=S(u)+Q\left(N_{f}(u)\right)+K\left(\varphi^{-1}\left[H\left(N_{f}(u)-Q\left(N_{f}(u)\right)\right)+\varphi(S(u))\right]\right) .
$$

Comparando o lema acima com o Lema 3.3.4, podemos observar que a construção do operador $M_{1}$ é a mesma, levando em conta, por outro lado, que o operador $\varphi^{-1}$ satisfaz hipóteses diferentes nos dois lemas. A demonstração do lema acima poderia ser facilmente desenvolvida no mesmo espírito da demonstração do Lema 3.3.4 e é portanto omitida.

A partir deste ponto $M_{1}$ é chamado operador ponto fixo associado ao problema (3.31).

Conseguimos provar o seguinte lema por um argumento clássico, utilizando o Teorema de ArzelàAscoli. A demonstração usa o mesmo raciocínio do Lema 3.4.8. Contudo, apresentamos aqui a prova, para ajudar o leitor.

Lema 3.5.2. O operador $M_{1}: C^{1} \longrightarrow C^{1}$ é completamente contínuo.

Demonstração. Seja $\Lambda$ um conjunto limitado de $C^{1}$. Então, existe $\rho>0$ tal que

$$
\|u\|_{1} \leq \rho
$$

para todo $u \in \Lambda$. Queremos mostrar que $\overline{M_{1}(\Lambda)} \subset C^{1}$ é um conjunto compacto. Para tanto, tome $\left(v_{n}\right)$ uma sequência em $M_{1}(\Lambda)$ e $\left(u_{n}\right)$ uma sequência em $\Lambda$ tal que $v_{n}=M_{1}\left(u_{n}\right)$. Da equação (3.32), segue que existe $L>0$ tal que

$$
\left\|N_{f}\left(u_{n}\right)\right\|_{\infty} \leq L
$$

para todo $n \in \mathbb{N}$. Daí se segue que

$$
\left\|H\left(N_{f}\left(u_{n}\right)-Q\left(N_{f}\left(u_{n}\right)\right)\right)\right\|_{\infty} \leq 2 L T .
$$

Portanto, a sequência $\left(H\left(N_{f}\left(u_{n}\right)-Q\left(N_{f}\left(u_{n}\right)\right)\right)\right)$ é limitada em $C$. Além disso, para todo $n \in \mathbb{N}$ e para $t, t_{1} \in[0, T]$, segue que

$$
\begin{aligned}
& \left|H\left(N_{f}\left(u_{n}\right)-Q\left(N_{f}\left(u_{n}\right)\right)\right)(t)-H\left(N_{f}\left(u_{n}\right)-Q\left(N_{f}\left(u_{n}\right)\right)\right)\left(t_{1}\right)\right| \\
& \leq\left|\int_{t_{1}}^{t} N_{f}\left(u_{n}\right)(s) d s\right|+\left|\int_{t_{1}}^{t} Q\left(N_{f}\left(u_{n}\right)\right)(s) d s\right| \\
& \leq L\left|t-t_{1}\right|+\left|t-t_{1}\right|\left\|Q\left(N_{f}\left(u_{n}\right)\right)\right\|_{\infty} \\
& \leq L\left|t-t_{1}\right|+L\left|t-t_{1}\right| \\
& \leq 2 L\left|t-t_{1}\right|
\end{aligned}
$$


o que implica que a sequência $\left(H\left(N_{f}\left(u_{n}\right)-Q\left(N_{f}\left(u_{n}\right)\right)\right)\right)$ é equicontínua. Assim, usando o Teorema de Arzelà-Ascoli, $\left(H\left(N_{f}\left(u_{n}\right)-Q\left(N_{f}\left(u_{n}\right)\right)\right)\right)$ possui uma subsequência convergente em $C$, que vamos chamar de

$$
\left(H\left(N_{f}\left(u_{n_{j}}\right)-Q\left(N_{f}\left(u_{n_{j}}\right)\right)\right)\right) .
$$

Então, passando a uma subsequência, se necessário, temos que a sequência

$$
\left(H\left(N_{f}\left(u_{n_{j}}\right)-Q\left(N_{f}\left(u_{n_{j}}\right)\right)\right)+\varphi\left(S\left(u_{n_{j}}\right)\right)\right)
$$

é convergente em $C$. Sabendo que $\varphi^{-1}: C \longrightarrow C$ é contínua e que

$$
M_{1}\left(u_{n_{j}}\right)^{\prime}=\varphi^{-1}\left[H\left(N_{f}\left(u_{n_{j}}\right)-Q\left(N_{f}\left(u_{n_{j}}\right)\right)\right)+\varphi\left(S\left(u_{n_{j}}\right)\right)\right],
$$

segue que $\left(M_{1}\left(u_{n_{j}}\right)^{\prime}\right)$ é convergente em $C$. Portanto, passando-se a uma subsequência, se necessário, obtemos que $\left(v_{n_{j}}\right)=\left(M_{1}\left(u_{n_{j}}\right)\right)$ é convergente em $C^{1}$. Para finalizar a prova, suponha $\left(v_{n}\right) \subseteq \overline{M_{1}(\Lambda)}$. Seja $\left(z_{n}\right) \subseteq M_{1}(\Lambda)$ tal que

$$
\lim _{n \rightarrow \infty}\left\|z_{n}-v_{n}\right\|_{1}=0 .
$$

Seja também $\left(z_{n_{j}}\right)$ uma subsequência de $\left(z_{n}\right)$ que converge para $z$. Segue que $z \in \overline{M_{1}(\Lambda)}$ e $\left(v_{n_{j}}\right)$ converge para $z$. Portanto, o resultado está provado.

Como feito no estudo do primeiro sistema (3.1), também aqui introduzimos uma família de problemas dependendo de um parametro real $\lambda$. Relembrando o operador de Nemytskii associado à $f$, ou seja,

$$
N_{f}(u)(t)=f\left(t, u(t), u^{\prime}(t)\right),
$$

para $\lambda \in[0,1]$, consideramos a família de problemas de valor na fronteira

$$
\left\{\begin{array}{l}
\left(\varphi\left(u^{\prime}\right)\right)^{\prime}=\lambda N_{f}(u)+(1-\lambda) Q\left(N_{f}(u)\right) \\
u(T)=u^{\prime}(0)=u^{\prime}(T) .
\end{array}\right.
$$

Note que (3.33) coincide com (3.31) quando $\lambda=1$. Para cada $\lambda \in[0,1]$, definimos o operador ponto fixo $M(\lambda, \cdot)$ sobre $C^{1}$ associado ao sistema (3.33), onde $M$ é definido em $[0,1] \times C^{1}$ por

$$
M(\lambda, u)=S(u)+Q\left(N_{f}(u)\right)+K\left(\varphi^{-1}\left[\lambda H\left(N_{f}(u)-Q\left(N_{f}(u)\right)\right)+\varphi(S(u))\right]\right) .
$$

Utilizando um argumento análogo ao Lema 3.5.1, conseguimos mostrar que podemos escrever (3.33) na forma equivalente $M(\lambda, u)=u$. Além disso, seguindo o mesmo raciocínio usado no Lema 3.5.2, é possível provar que $M$ é completamente contínuo.

Antes de provarmos o principal resultado da seção, definimos a seguinte aplicação contínua:

$$
G: \mathbb{R}^{2} \longrightarrow \mathbb{R}^{2},(a, b) \longmapsto\left(a T+b T^{2}-b T-\frac{1}{T} \int_{0}^{T} f(t, a+b t, b) d t, b-a-b T\right) .
$$

Finalmente, temos o resultado principal desta seção que mostra a existência de pelo menos uma solução de (3.31).

Teorema 3.5.3. Seja $\Omega$ um subconjunto aberto limitado de $C^{1}$. Consideramos a identificação natural $(a, b) \approx a+b t$ de $\mathbb{R}^{2}$ com as funções afins de $C^{1}$. Suponhamos sejam verificadas as seguintes condições.

1. Para cada $\lambda \in(0,1)$, o problema

$$
\left\{\begin{array}{l}
\left(\varphi\left(u^{\prime}\right)\right)^{\prime}=\lambda N_{f}(u) \\
u(T)=u^{\prime}(0)=u^{\prime}(T)
\end{array}\right.
$$

não tem solução em $\partial \Omega$. 
2. A equação

$$
G(a, b)=(0,0)
$$

não tem solução em $\partial \Omega \cap \mathbb{R}^{2}$.

3. O grau de Brouwer

$$
\operatorname{deg}_{B}\left(G, \Omega \cap \mathbb{R}^{2}, 0\right) \neq 0
$$

Então, o problema (3.31) tem uma solução.

Demonstração. Para $\lambda \in(0,1]$, se $u$ é solução de (3.34), então $Q\left(N_{f}(u)\right)=0$, portanto $u$ é solução de (3.33). Por outro lado, ainda para $\lambda \in(0,1]$, se $u$ é solução de (3.33) e tendo em conta que

$$
Q\left(\lambda N_{f}(u)+(1-\lambda) Q\left(N_{f}(u)\right)\right)=Q\left(N_{f}(u)\right),
$$

temos que $Q\left(N_{f}(u)\right)=0$. Desta maneira, $u$ é solução de (3.34). Por conseguinte, os problemas (3.33) e (3.34) possuem o mesmo conjunto de soluções para $\lambda \in(0,1]$.

Suponha que (3.33) não tenha solução para $\lambda=1$ (do contrario o teorema estaria trivialmente provado). Portanto, (3.33) não tem solução em $\partial \Omega$ para todo $\lambda \in(0,1]$. Para $\lambda=0$, (3.33) é equivalente ao sistema

$$
\left\{\begin{array}{l}
\left(\varphi\left(u^{\prime}\right)\right)^{\prime}=Q\left(N_{f}(u)\right) \\
u(T)=u^{\prime}(0)=u^{\prime}(T) .
\end{array}\right.
$$

Se $u$ é solução de (3.36), então $Q\left(N_{f}(u)\right)=0$. Portanto, $u$ satisfaz a equação

$$
\left(\varphi\left(u^{\prime}(t)\right)\right)^{\prime}=0, u(T)=u^{\prime}(0)=u^{\prime}(T)
$$

para todo $t \in[0, T]$. Desta forma, concluimos que $u$ é uma função de tipo $u(t)=a+b t, a=b-b T$. Como $Q\left(N_{f}(u)\right)=0$, temos

$$
\frac{1}{T} \int_{0}^{T} f(t, a+b t, b) d t=0
$$

Utilizando a condição (3.35) da hipótese, podemos afirmar que $u \notin \partial \Omega$. Sendo assim, provamos que o problema (3.33) não tem solução em $\partial \Omega$ para todo $\lambda \in[0,1]$. Portanto, para cada $\lambda \in[0,1]$, o grau de Leray-Schauder $\operatorname{deg}_{L S}(I-M(\lambda, \cdot), \Omega, 0)$ está bem definido. Logo, usando a propriedade de invariância homotópica, podemos afirmar que

$$
\operatorname{deg}_{L S}(I-M(0, \cdot), \Omega, 0)=\operatorname{deg}_{L S}(I-M(1, \cdot), \Omega, 0) .
$$

Por outro lado,

$$
\operatorname{deg}_{L S}(I-M(0, \cdot), \Omega, 0)=\operatorname{deg}_{L S}\left(I-\left(S+Q N_{f}+K S\right), \Omega, 0\right) .
$$

Além disso, o conjunto imagem da aplicação

$$
u \longmapsto S(u)+Q\left(N_{f}(u)\right)+K(S(u))
$$

está contido no subespaço das funções afins isomorfo a $\mathbb{R}^{2}$. Assim, usando a propriedade de redução finita dimensional do grau de Leray-Schauder, temos

$$
\begin{aligned}
& \operatorname{deg}_{L S}\left(I-\left(S+Q N_{f}+K S\right), \Omega, 0\right) \\
& =\operatorname{deg}_{B}\left(I-\left(S+Q N_{f}+K S\right) \mid \overline{\Omega \cap \mathbb{R}^{2}}, \Omega \cap \mathbb{R}^{2}, 0\right) \\
& =\operatorname{deg}_{B}\left(G, \Omega \cap \mathbb{R}^{2}, 0\right) \neq 0 .
\end{aligned}
$$

Então, $\operatorname{deg}_{L S}(I-M(1, \cdot), \Omega, 0) \neq 0$. Portanto, existe $u \in \Omega$ tal que $M_{1}(u)=u$, o que significa que $u$ é solução de (3.31).

Nosso seguinte teorema estabelece condições para garantir limitações a priori para as soluções do problema (3.34). Este resultado foi inspirado em [8] (Bereanu e Mawhin). 
Teorema 3.5.4. Seja $f$ uma função contínua que satisfaz as seguintes condições.

1. Existe uma função $c \in C$ tal que

$$
f(t, x, y) \geq c(t)
$$

para todo $(t, x, y) \in[0, T] \times \mathbb{R} \times \mathbb{R}$.

2. Existem $M_{1}<M_{2}$ tais que para toda $u \in C^{1}$

$$
\int_{0}^{T} f\left(t, u(t), u^{\prime}(t)\right) d t \neq 0 \quad \text { se } u_{m}^{\prime} \geq M_{2}
$$

ou

$$
\int_{0}^{T} f\left(t, u(t), u^{\prime}(t)\right) d t \neq 0 \quad \text { se } \quad u_{M}^{\prime} \leq M_{1} .
$$

Se $(\lambda, u) \in(0,1) \times C^{1}$ é tal que u é solução de (3.34), então

$$
\|u\|_{1}<r(2+T)
$$

onde $r=\max \left\{\left|\varphi^{-1}\left(L+2\left\|c^{-}\right\|_{L^{1}}\right)\right|,\left|\varphi^{-1}\left(-L-2\left\|c^{-}\right\|_{L^{1}}\right)\right|\right\} \quad$ e $L=\max \left\{\left|\varphi\left(M_{2}\right)\right|,\left|\varphi\left(M_{1}\right)\right|\right\}$.

Demonstração. Para $\lambda \in(0,1)$, se $u$ é solução do problema (3.34), então para todo $t \in[0, T]$,

$$
\left(\varphi\left(u^{\prime}(t)\right)\right)^{\prime}=\lambda N_{f}(u)(t), u^{\prime}(0)=u^{\prime}(T)=u(T)
$$

$\mathrm{e}$

$$
\int_{0}^{T} f\left(t, u(t), u^{\prime}(t)\right) d t=0 .
$$

Usando a hipótese 2 acima, temos

$$
u_{m}^{\prime}<M_{2} \text { e } u_{M}^{\prime}>M_{1} .
$$

Portanto, existe $\omega \in[0, T]$ tal que $M_{1}<u^{\prime}(\omega)<M_{2}$ e

$$
\int_{\omega}^{t}\left(\varphi\left(u^{\prime}(s)\right)\right)^{\prime} d s=\lambda \int_{\omega}^{t} N_{f}(u)(s) d s .
$$

Assim,

$$
\left|\varphi\left(u^{\prime}(t)\right)\right| \leq\left|\varphi\left(u^{\prime}(\omega)\right)\right|+\int_{0}^{T}\left|f\left(s, u(s), u^{\prime}(s)\right)\right| d s,
$$

com

$$
\int_{0}^{T}\left|f\left(s, u(s), u^{\prime}(s)\right)\right| d s \leq \int_{0}^{T} f\left(s, u(s), u^{\prime}(s)\right) d s+2 \int_{0}^{T} c^{-}(s) d s,
$$

onde, lembramos, $c^{-}=\max \{-c, 0\}$. Por conseguinte,

$$
\left|\varphi\left(u^{\prime}(t)\right)\right| \leq\left|\varphi\left(u^{\prime}(\omega)\right)\right|+2\left\|c^{-}\right\|_{L^{1}}
$$

para todo $t \in[0, T]$. Logo,

$$
\left|\varphi\left(u^{\prime}(t)\right)\right|<L+2\left\|c^{-}\right\|_{L^{1}},
$$

onde $L=\max \left\{\left|\varphi\left(M_{2}\right)\right|,\left|\varphi\left(M_{1}\right)\right|\right\}$ e $t \in[0, T]$. Desta forma, concluímos que

$$
\left\|u^{\prime}\right\|_{\infty}<r
$$

onde $r=\max \left\{\left|\varphi^{-1}\left(L+2\left\|c^{-}\right\|_{L^{1}}\right)\right|, \quad\left|\varphi^{-1}\left(-L-2\left\|c^{-}\right\|_{L^{1}}\right)\right|\right\}$. Usando o fato de que $u^{\prime}(0)=u(T)$, deduzimos que

$$
|u(t)| \leq|u(T)|+\int_{0}^{T}\left|u^{\prime}(s)\right| d s<r+r T \quad(t \in[0, T]),
$$

e, portanto,

$$
\|u\|_{1}=\|u\|_{\infty}+\left\|u^{\prime}\right\|_{\infty}<r+r T+r=r(2+T) .
$$

Assim completamos a demonstração. 
Nosso próximo resultado nos permite estabelecer a existência de pelo menos uma solução do problema (3.31).

Teorema 3.5.5. Seja $f$ uma função contínua que atende as condições do Teorema 3.5.4. Suponha que as seguintes condiçôes sejam satisfeitas para algum $\rho \geq r(2+T)$.

1. A equação

$$
G(a, b)=(0,0)
$$

não tem solução em $\partial B_{\rho}(0) \cap \mathbb{R}^{2}$.

2. O grau de Brouwer

$$
\operatorname{deg}_{B}\left(G, B_{\rho}(0) \cap \mathbb{R}^{2}, 0\right) \neq 0 .
$$

Então, o problema (3.31) tem uma solução.

Demonstração. Para $\lambda \in(0,1)$, se $u$ é solução do problema (3.34), então pelo Teorema 3.5.4, temos

$$
\|u\|_{1}=\|u\|_{\infty}+\left\|u^{\prime}\right\|_{\infty}<r+r T+r=r(2+T),
$$

onde $r=\max \left\{\left|\varphi^{-1}\left(L+2\left\|c^{-}\right\|_{L^{1}}\right)\right|,\left|\varphi^{-1}\left(-L-2\left\|c^{-}\right\|_{L^{1}}\right)\right|\right\}$. Usando o Teorema 3.5.3 com $\Omega=$ $B_{\rho}(0)$, completamos a demonstração.

O seguinte exemplo é uma aplicacão do Teorema 3.5.5.

Exemplo 3.5.6. Vamos considerar o problema

$$
\left(u^{\prime 3}\right)^{\prime}=\frac{e^{u^{\prime}}}{2}-1, \quad u(T)=u^{\prime}(0)=u^{\prime}(T) .
$$

Para $M_{1}=-1, M_{2}=1$ e $c(t)=-1$ para todo $t \in[0, T]$. O problema (3.38) tem ao menos uma solucão para $\rho \geq(1+2 T)^{1 / 3}(2+T)$.

\subsection{Problemas do tipo misto generalizado em $\mathbb{R}$}

Nesta seção, nosso objeto de estudo será uma classe de equações diferenciais com condições de fronteira da forma

$$
u^{\prime}(0)=u(0), u^{\prime}(T)=b u^{\prime}(0)
$$

para $b \in \mathbb{R}, \quad b \neq 0$.

No artigo [10] Bouches e Mawhin estudam a existência de ao menos uma solução do problema de tipo

$$
\left\{\begin{array}{l}
(\varphi(u))^{\prime}=f(t, u) \\
u(T)=b u(0),
\end{array}\right.
$$

onde $\varphi: \mathbb{R} \longrightarrow(-a, a)$ é um homeomorfismo tal que $\varphi(0)=0, f:[0, T] \times \mathbb{R} \longrightarrow \mathbb{R}$ uma função contínua e $b \in \mathbb{R}, \quad b \neq 0$.

Inspirados por [10], investigamos a existência de soluções para o seguinte sistema que não encontramos na literatura:

$$
\left\{\begin{array}{l}
\left(\varphi\left(u^{\prime}\right)\right)^{\prime}=f\left(t, u, u^{\prime}\right) \\
u^{\prime}(0)=u(0), u^{\prime}(T)=b u^{\prime}(0)
\end{array}\right.
$$

onde $\varphi: \mathbb{R} \longrightarrow \mathbb{R}$ é um homeomorfismo tal que $\varphi(0)=0, \quad f:[0, T] \times \mathbb{R} \times \mathbb{R} \longrightarrow \mathbb{R}$ uma função contínua e $b \in \mathbb{R}, \quad b \neq 0$. 
Diremos que $u:[0, T] \longrightarrow \mathbb{R}$ de classe $C^{1}$ é uma solução do problema (3.40) quando as condições seguintes forem satisfeitas: $u$ atende as condições de fronteira e a função $\varphi\left(u^{\prime}\right)$ é de classe $C^{1} \mathrm{e}$ satisfaz $\left(\varphi\left(u^{\prime}(t)\right)\right)^{\prime}=f\left(t, u(t), u^{\prime}(t)\right)$ para todo $t$ em $[0, T]$.

O procedimento adotado para mostrar a existência de pelo menos uma solução de (3.40) é transformar o sistema em um problema de ponto fixo para um operador definido em $C^{1}$. Em seguida, sob certas condições, usamos o grau de Leray-Schauder e o grau de Brouwer. Vamos aqui apresentar esta abordagem.

Como nos casos anteriores, $\varphi^{-1}$ é entendida como uma função $\varphi^{-1}: C \longrightarrow C$ dada por $\varphi^{-1}(v)(t)=\varphi^{-1}(v(t))$. É claro que $\varphi^{-1}$ é contínua e envia conjuntos limitados em conjuntos limitados.

Diante do exposto acima consideramos a seguinte aplicação:

$$
M_{1}(u)=Q\left(N_{f}(u)\right)-\frac{B_{\varphi, b}(P u)}{T}+H\left(\varphi^{-1}\left[\varphi(P u)+H\left(N_{f}(u)-Q\left(N_{f}(u)\right)\right)+\frac{\Psi B_{\varphi, b}(P u)}{T}\right]\right)+P(u),
$$

onde $\Psi$ denota a função que envia $t$ em $t, \mathbb{R}$ é identificado com o subespaço de $C^{1}$ das funções constantes e $B_{\varphi, b}: \mathbb{R} \longrightarrow \mathbb{R}$ é definido por $x \longmapsto B_{\varphi, b}(x)=\varphi(b x)-\varphi(x)$. Lembramos que os espaços $C$ e $C^{1}$ foram definidos na seção 3.1 .

Usando as propriedades do operador $M_{1}$ juntamente com o Teorema de Arzelà-Ascoli (Teorema 2.3.5), temos o seguinte lema. A demonstração é apresentada, mesmo sendo o resultado análogo a vários resultados anteriores.

Lema 3.6.1. O operador $M_{1}: C^{1} \longrightarrow C^{1}$ é completamente contínuo.

Demonstração. Seja $\Lambda$ um conjunto limitado de $C^{1}$. Então, existe $\rho>0$ tal que

$$
\|u\|_{1} \leq \rho
$$

para todo $u \in \Lambda$. Queremos mostrar que $\overline{M_{1}(\Lambda)} \subset C^{1}$ é um conjunto compacto. Para tanto, tome $\left(v_{n}\right)$ uma sequência em $M_{1}(\Lambda)$ e $\left(u_{n}\right)$ uma sequência em $\Lambda$ tal que $v_{n}=M_{1}\left(u_{n}\right)$. Da fórmula (3.41), segue que existe $L>0$ tal que

$$
\left\|N_{f}\left(u_{n}\right)\right\|_{\infty} \leq L
$$

para todo $n \in \mathbb{N}$. Daí se segue que

$$
\left\|H\left(N_{f}\left(u_{n}\right)-Q\left(N_{f}\left(u_{n}\right)\right)\right)\right\|_{\infty} \leq 2 L T .
$$

Portanto, a sequência $\left(H\left(N_{f}\left(u_{n}\right)-Q\left(N_{f}\left(u_{n}\right)\right)\right)\right)$ é limitada em $C$. Além disso, para todo $n \in \mathbb{N}$ e para $t, t_{1} \in[0, T]$, segue que

$$
\begin{aligned}
& \left|H\left(N_{f}\left(u_{n}\right)-Q\left(N_{f}\left(u_{n}\right)\right)\right)(t)-H\left(N_{f}\left(u_{n}\right)-Q\left(N_{f}\left(u_{n}\right)\right)\right)\left(t_{1}\right)\right| \\
& \leq\left|\int_{t_{1}}^{t} N_{f}\left(u_{n}\right)(s) d s\right|+\left|\int_{t_{1}}^{t} Q\left(N_{f}\left(u_{n}\right)\right)(s) d s\right| \\
& \leq L\left|t-t_{1}\right|+\left|t-t_{1}\right|\left\|Q\left(N_{f}\left(u_{n}\right)\right)\right\|_{\infty} \\
& \leq L\left|t-t_{1}\right|+L\left|t-t_{1}\right| \\
& \leq 2 L\left|t-t_{1}\right|
\end{aligned}
$$

o que implica que a sequência $\left(H\left(N_{f}\left(u_{n}\right)-Q\left(N_{f}\left(u_{n}\right)\right)\right)\right)$ é equicontínua. Assim, usando o Teorema de Arzelà-Ascoli (Teorema 2.3.5), $\left(H\left(N_{f}\left(u_{n}\right)-Q\left(N_{f}\left(u_{n}\right)\right)\right)\right)$ possui uma subsequência convergente em $C$, que vamos chamar de

$$
\left(H\left(N_{f}\left(u_{n_{j}}\right)-Q\left(N_{f}\left(u_{n_{j}}\right)\right)\right)\right) .
$$

Então, passando a uma subsequência, se necessário, temos que a sequência

$$
\left(H\left(N_{f}\left(u_{n_{j}}\right)-Q\left(N_{f}\left(u_{n_{j}}\right)\right)\right)+\frac{\Psi B_{\varphi, b}\left(P u_{n_{j}}\right)}{T}+\varphi\left(P\left(u_{n_{j}}\right)\right)\right)
$$


é convergente em $C$. Sabendo que $\varphi^{-1}: C \longrightarrow C$ é contínua e que

$$
M_{1}\left(u_{n_{j}}\right)^{\prime}=\varphi^{-1}\left[\left(H\left(N_{f}\left(u_{n_{j}}\right)-Q\left(N_{f}\left(u_{n_{j}}\right)\right)\right)+\frac{\Psi B_{\varphi, b}\left(P u_{n_{j}}\right)}{T}+\varphi\left(P\left(u_{n_{j}}\right)\right)\right)\right],
$$

segue que a sequência $\left(M_{1}\left(u_{n_{j}}\right)^{\prime}\right)$ é convergente em $C$. Portanto, de novo passando a uma subsequência, se necessário, obtemos que $\left(v_{n_{j}}\right)=\left(M_{1}\left(u_{n_{j}}\right)\right)$ é convergente em $C^{1}$. Para finalizar a prova, suponha $\left(v_{n}\right) \subseteq \overline{M_{1}(\Lambda)}$. Seja $\left(z_{n}\right) \subseteq M_{1}(\Lambda)$ tal que

$$
\lim _{n \rightarrow \infty}\left\|z_{n}-v_{n}\right\|_{1}=0
$$

Seja também $\left(z_{n_{j}}\right)$ uma subsequência de $\left(z_{n}\right)$ que converge para $z$. Segue que $z \in \overline{M_{1}(\Lambda)}$ e $\left(v_{n_{j}}\right)$ converge para $z$. Portanto, o resultado está provado.

O seguinte lema estabelece uma relação direta do problema (3.40) com o operador $M_{1}$. Alguns detalhes técnicos da demonstração do resultado nos sugerem que seja oportuno apresentá-la.

Lema 3.6.2. $u \in C^{1}$ é uma solução de (3.40) se, e somente se, u é um ponto fixo do operador $M_{1}$.

Demonstração. Seja $u \in C^{1}$. Obtemos as seguintes equivalencias:

$$
\begin{aligned}
& \left(\varphi\left(u^{\prime}\right)\right)^{\prime}=N_{f}(u), u^{\prime}(T)=b u^{\prime}(0), u^{\prime}(0)=u(0) \\
\Leftrightarrow & \left(\varphi\left(u^{\prime}\right)\right)^{\prime}=N_{f}(u)-\left(Q\left(N_{f}(u)\right)-\frac{B_{\varphi, b}\left(u^{\prime}(0)\right)}{T}\right) \\
& Q\left(N_{f}(u)\right)-\frac{B_{\varphi, b}\left(u^{\prime}(0)\right)}{T}=0, u^{\prime}(0)=u(0) \\
\Leftrightarrow & \varphi\left(u^{\prime}\right)=H\left(N_{f}(u)-Q\left(N_{f}(u)\right)\right)+\frac{\Psi B_{\varphi, b}\left(u^{\prime}(0)\right)}{T}+\varphi\left(u^{\prime}(0)\right), \\
& Q\left(N_{f}(u)\right)-\frac{B_{\varphi, b}\left(u^{\prime}(0)\right)}{T}=0, u^{\prime}(0)=u(0) \\
\Leftrightarrow & u^{\prime}=\varphi^{-1}\left[H\left(N_{f}(u)-Q\left(N_{f}(u)\right)+\frac{\Psi B_{\varphi, b}\left(u^{\prime}(0)\right)}{T}+\varphi\left(u^{\prime}(0)\right)\right]\right. \\
& Q\left(N_{f}(u)\right)-\frac{B_{\varphi, b}\left(u^{\prime}(0)\right)}{T}=0, u^{\prime}(0)=u(0) \\
\Leftrightarrow & u=H\left(\varphi^{-1}\left[H\left(N_{f}(u)-Q\left(N_{f}(u)\right)\right)+\frac{\Psi B_{\varphi, b}\left(u^{\prime}(0)\right)}{T}+\varphi\left(u^{\prime}(0)\right)\right]\right)+u(0), \\
& Q\left(N_{f}(u)\right)-\frac{B_{\varphi, b}\left(u^{\prime}(0)\right)}{T}=0, u^{\prime}(0)=u(0) \\
\Leftrightarrow & u=H\left(\varphi^{-1}\left[H\left(N_{f}(u)-Q\left(N_{f}(u)\right)\right)+\frac{\Psi B_{\varphi, b}(u(0))}{T}+\varphi(u(0))\right]\right)+u(0), \\
& Q\left(N_{f}(u)\right)-\frac{B_{\varphi, b}(u(0))}{T}=0 \\
\Leftrightarrow & u=Q\left(N_{f}(u)\right)-\frac{B_{\varphi, b}(P u)}{T}+H\left(\varphi^{-1}\left[H\left(N_{f}(u)-Q\left(N_{f}(u)\right)\right)+\frac{\Psi B_{\varphi, b}(P u)}{T}+\varphi(P u)\right]\right)+P u .
\end{aligned}
$$

Observação 3.6.3. Observe que $u^{\prime}(T)=b u^{\prime}(0)$ se, e somente se, $Q\left(N_{f}(u)\right)=\frac{B_{\varphi, b}\left(u^{\prime}(0)\right)}{T}$.

A partir deste ponto, $M_{1}$ será chamado operador ponto fixo associado ao sistema (3.40).

Queremos aplicar o grau de Leray-Schauder ao operador $M_{1}$. Introduzimos uma família de problemas de valor na fronteira dependendo de um parametro real $\lambda$. Para $\lambda \in[0,1]$, consideramos a família

$$
\left\{\begin{array}{l}
\left(\varphi\left(u^{\prime}\right)\right)^{\prime}=\lambda N_{f}(u)+(1-\lambda) Q\left(N_{f}(u)\right) \\
u^{\prime}(0)=u(0), u^{\prime}(T)=b u^{\prime}(0) .
\end{array}\right.
$$

Note que (3.42) coincide com (3.40) quando $\lambda=1$. Assim, para cada $\lambda \in[0,1]$, o operador ponto fixo associado ao problema (3.42) pelo Lema 3.6.2 é o operador $M(\lambda, \cdot)$, onde $M$ é definido em $[0,1] \times C^{1}$ por 
$M(\lambda, u)=Q\left(N_{f}(u)\right)-\frac{B_{\varphi, b}(P u)}{T}+H\left(\varphi^{-1}\left[\varphi(P u)+\lambda H\left(N_{f}(u)-Q\left(N_{f}(u)\right)\right)+\frac{\Psi B_{\varphi, b}(P u)}{T}\right]\right)+P(u)$.

Utilizando argumentos análogos aos Lemas 3.6.2 e 3.6.1, conseguimos mostrar que podemos escrever (3.42) de forma equivalente $M(\lambda, u)=u$ e que $M$ é completamente contínuo, respectivamente.

A fim de provar a existência de ao menos uma solução de (3.40) vamos introduzir um novo sistema

$$
\left\{\begin{array}{l}
\left(\varphi\left(u^{\prime}\right)\right)^{\prime}=\lambda Q\left(N_{f}(u)\right) \\
\int_{0}^{T} f\left(t, u(t), u^{\prime}(t)\right) d t=\varphi(b u(0))-\varphi(u(0)), u^{\prime}(0)=u(0) .
\end{array}\right.
$$

Por outro lado, vamos considerar a seguinte aplicação $Z$ definida em $[0,1] \times C^{1}$ por

$$
Z(\lambda, u)=P(u)+Q\left(N_{f}(u)\right)-\frac{B_{\varphi, b}(P u)}{T}+H\left(\varphi^{-1}\left[\lambda \frac{\Psi B_{\varphi, b}(P u)}{T}+\varphi(P u)\right]\right),
$$

onde $Z(1, \cdot)$ coincide com o operador $M(0, \cdot)$.

A seguir, apresentamos duas propriedades importantes de $Z$.

Lema 3.6.4. Se $u \in C^{1}$ é ponto fixo do operador $Z(\lambda, \cdot)$ para $\lambda \in[0,1]$, então u é solução de (3.43).

Demonstração. Para $\lambda \in[0,1]$, se $u \in C^{1}$ é ponto fixo do operador $Z(\lambda, \cdot)$, então $u=Z(\lambda, u)$. Avaliando a função $u$ em 0 , segue que

$$
\int_{0}^{T} f\left(t, u(t), u^{\prime}(t)\right) d t=\varphi(b u(0))-\varphi(u(0)) .
$$

Derivando $u$, obtemos

$$
u^{\prime}(t)=\varphi^{-1}\left[t \lambda \frac{\varphi(b u(0))-\varphi(u(0))}{T}+\varphi(u(0))\right]
$$

para todo $t \in[0, T]$. Aplicando $\varphi$, segue que

$$
\varphi\left(u^{\prime}(t)\right)=t \lambda \frac{\varphi(b u(0))-\varphi(u(0))}{T}+\varphi(u(0)) .
$$

Agora, derive novamente e conclua que

$$
\left(\varphi\left(u^{\prime}(t)\right)\right)^{\prime}=\lambda \frac{\varphi(b u(0))-\varphi(u(0))}{T}=\lambda Q\left(N_{f}(u)\right)
$$

para todo $t \in[0, T]$.

Por outro lado, usando (3.45) para $t=0$, obtemos $u^{\prime}(0)=u(0)$, finalizando a demonstração do lema.

Lema 3.6.5. O operador $Z:[0,1] \times C^{1} \longrightarrow C^{1}$ é completamente contínuo.

Demonstração. Seja $\Lambda$ um conjunto limitado de $[0,1] \times C^{1}$. Então, existe $\rho>0$ tal que

$$
\|(\lambda, u)\|=\max \left\{\|\lambda\|,\|u\|_{1}\right\} \leq \rho
$$

para todo $(\lambda, u) \in \Lambda$. Queremos mostrar que $\overline{Z(\Lambda)} \subset C^{1}$ é um conjunto compacto. Para tanto, tome $\left(v_{n}\right)$ uma sequência em $Z(\Lambda)$ e $\left(\lambda_{n}, u_{n}\right)$ uma sequência em $\Lambda$ tal que $v_{n}=Z\left(\lambda_{n}, u_{n}\right)$. De (3.46) e sabendo que $\varphi$ é um homeomorfismo, deduzimos que existe uma constante $L>0$ tal que

$$
\left\|\lambda_{n} \frac{B_{\varphi, b}\left(P u_{n}\right)}{T}\right\|_{\infty} \leq L
$$


para todo $n \in \mathbb{N}$. Logo, a sequência $\left(\lambda_{n} \frac{\Psi B_{\varphi, b}\left(P u_{n}\right)}{T}\right)$ é limitada em $C$. Além disso, para todo $n \in \mathbb{N}$ e para $t, t_{1} \in[0, T]$, segue que

$$
\begin{aligned}
& \left|\lambda_{n} \frac{t B_{\varphi, b}\left(P u_{n}\right)(t)}{T}-\lambda_{n} \frac{t_{1} B_{\varphi, b}\left(P u_{n}\right)\left(t_{1}\right)}{T}\right| \\
& \leq\left|\lambda_{n} t \frac{\varphi\left(b u_{n}(0)\right)-\varphi\left(u_{n}(0)\right)}{T}-\lambda_{n} t_{1} \frac{\varphi\left(b u_{n}(0)\right)-\varphi\left(u_{n}(0)\right)}{T}\right| \\
& \leq\left|\left(t-t_{1}\right)\left(\lambda_{n} \frac{\varphi\left(b u_{n}(0)\right)-\varphi\left(u_{n}(0)\right)}{T}\right)\right| \\
& \leq L\left|t-t_{1}\right|,
\end{aligned}
$$

o que implica que a sequência $\left(\lambda_{n} \frac{\Psi B_{\varphi, b}\left(P u_{n}\right)}{T}\right)$ é equicontínua. Assim, usando o Teorema de ArzelàAscoli (Teorema 2.3.5), $\left(\lambda_{n} \frac{\Psi B_{\varphi, b}\left(P u_{n}\right)}{T}\right)$ possui uma subsequência convergente em $C$, que vamos chamar de

$$
\left(\lambda_{n_{j}} \frac{\Psi B_{\varphi, b}\left(P u_{n_{j}}\right)}{T}\right) .
$$

Então, passando a uma subsequência, se necessário, temos que a sequência

$$
\left(\lambda_{n_{j}} \frac{\Psi B_{\varphi, b}\left(P u_{n_{j}}\right)}{T}+\varphi\left(P\left(u_{n_{j}}\right)\right)\right)
$$

é convergente em $C$. Usando a continuidade de $\varphi^{-1}: C \longrightarrow C$ e a igualdade seguinte:

$$
Z\left(\lambda_{n_{j}}, u_{n_{j}}\right)^{\prime}=\varphi^{-1}\left[\left(\lambda_{n_{j}} \frac{\Psi B_{\varphi, b}\left(P u_{n_{j}}\right)}{T}+\varphi\left(P\left(u_{n_{j}}\right)\right)\right)\right]
$$

segue que a sequência $\left(Z\left(\lambda_{n_{j}}, u_{n_{j}}\right)^{\prime}\right)$ é convergente em $C$ e, portanto, passando novamente a uma subsequência, se necessário, obtemos que $\left(v_{n_{j}}\right)=\left(Z\left(\lambda_{n_{j}}, u_{n_{j}}\right)\right)$ é convergente em $C^{1}$. Para finalizar a prova, suponha $\left(v_{n}\right) \subseteq \overline{Z(\Lambda)}$. Seja $\left(z_{n}\right) \subseteq Z(\Lambda)$ tal que

$$
\lim _{n \rightarrow \infty}\left\|z_{n}-v_{n}\right\|_{1}=0
$$

Seja também $\left(z_{n_{j}}\right)$ uma subsequência de $\left(z_{n}\right)$ que converge para $z$. Segue que $z \in \overline{Z(\Lambda)}$ e $\left(v_{n_{j}}\right)$ converge para $z$. Portanto, o resultado está provado.

Neste momento, estamos em condições de enunciar o principal resultado desta seção que mostra a existência de alguma solução do problema (3.40). Consideramos a seguinte aplicação contínua:

$$
G: \mathbb{R}^{2} \longrightarrow \mathbb{R}^{2}, \quad(x, y) \longmapsto\left(\frac{B_{\varphi, b}(x)}{T}-\frac{1}{T} \int_{0}^{T} f(t, x+y t, y) d t,-x+y\right) .
$$

Teorema 3.6.6. Seja $\Omega$ um subconjunto aberto limitado de $C^{1}$. Consideramos a identificação natural $(a, b) \approx a+b t$ de $\mathbb{R}^{2}$ com as funções afins de $C^{1}$. Suponhamos sejam verificadas as seguintes condições.

1. Se u é ponto fixo do operador $Z(\lambda, \cdot)$ para $\lambda \in[0,1]$, então u não pertence a $\partial \Omega$.

2. O grau de Brouwer

$$
\operatorname{deg}_{B}\left(G, \Omega \cap \mathbb{R}^{2}, 0\right) \neq 0 .
$$

3. Para cada $\lambda \in(0,1]$, (3.42) não tem solução em $\partial \Omega$.

Então, o problema (3.40) tem uma solução. 
Demonstração. Pelo Lema 3.6.5 e usando a hipótese 1 acima, temos que para $\lambda \in[0,1]$, o grau de Leray-Schauder $\operatorname{deg}_{L S}(I-Z(\lambda, \cdot), \Omega, 0)$ está bem definido. A invariância homotópica diz que

$$
\operatorname{deg}_{L S}(I-Z(1, \cdot), \Omega, 0)=\operatorname{deg}_{L S}(I-Z(0, \cdot), \Omega, 0) .
$$

Por outro lado,

$$
\operatorname{deg}_{L S}(I-Z(0, \cdot), \Omega, 0)=\operatorname{deg}_{L S}\left(I-\left(P+Q N_{f}-\frac{B_{\varphi, b} P}{T}+H P\right), \Omega, 0\right) .
$$

Além disso, o conjunto imagem da aplicação

$$
u \longrightarrow P(u)+Q\left(N_{f}(u)\right)-\frac{B_{\varphi, b}(P(u))}{T}+H(P(u))
$$

está contido no subespaço das funções afins isomorfo a $\mathbb{R}^{2}$. Assim, usando a propriedade de redução em dimensão finita do grau de Leray-Schauder [15, 27]

$$
\begin{aligned}
& \operatorname{deg}_{L S}\left(I-\left(P+Q N_{f}-\frac{B_{\varphi, b} P}{T}+H P\right), \Omega, 0\right) \\
& =\operatorname{deg}_{B}\left(I-\left(P+Q N_{f}-\frac{B_{\varphi, b} P}{T}+H P\right) \mid \overline{\Omega \cap \mathbb{R}^{2}}, \Omega \cap \mathbb{R}^{2}, 0\right) \\
& =\operatorname{deg}_{B}\left(G, \Omega \cap \mathbb{R}^{2}, 0\right) \neq 0 .
\end{aligned}
$$

Por outro lado, como $M(0, \cdot)$ coincide com $Z(1, \cdot), M$ é completamente contínuo e $(3.42)$ não tem solução em $\partial \Omega$ para $\lambda \in(0,1]$, então podemos concluir que, para cada $\lambda \in[0,1]$, $\operatorname{deg}_{L S}(I-$ $M(\lambda, \cdot), \Omega, 0)$ está bem definido. Logo, utilizando a propriedade de invariância homotópica, podemos afirmar que

$$
\operatorname{deg}_{L S}(I-M(1, \cdot), \Omega, 0)=\operatorname{deg}_{L S}(I-M(0, \cdot), \Omega, 0) .
$$

Por conseguinte, $\operatorname{deg}_{L S}(I-M(1, \cdot), \Omega, 0) \neq 0$. Portanto, existe $u \in \Omega$ tal que $M_{1}(u)=u$, o que significa que $u$ é solução de (3.40).

O problema (3.40) pode ser estudado colocando algumas condições particulares sobre a função $f$ (ou o homeomorfismo $\varphi$ no Teorema 3.6.6). Provamos os resultados seguintes.

Teorema 3.6.7. Seja $f$ uma função contínua. Suponha que as seguintes condições sejam satisfeitas.

1. Existem $M_{1}<M_{2}$ tais que para toda $u \in C^{1}$

$$
\int_{0}^{T} f\left(t, u(t), u^{\prime}(t)\right) d t-B_{\varphi, b}\left(u^{\prime}(0)\right) \neq 0 \quad \text { se } \quad u_{m}^{\prime} \geq M_{2}
$$

ou

$$
\int_{0}^{T} f\left(t, u(t), u^{\prime}(t)\right) d t-B_{\varphi, b}\left(u^{\prime}(0)\right) \neq 0 \text { se } u_{M}^{\prime} \leq M_{1} .
$$

2. Existe $h \in C$ tal que

$$
|f(t, x, y)| \leq h(t)
$$

para todo $x, y \in \mathbb{R}$ e todo $t \in[0, T]$.

3. Para um oportuno $\rho>0$, o grau de Brouwer

$$
\operatorname{deg}_{B}\left(G, B_{\rho}(0) \cap \mathbb{R}^{2}, 0\right) \neq 0 \text {, onde } G \text { é definida em }(3.47)
$$

Então, o problema (3.40) tem ao menos uma solução.

Demonstração. Para $\lambda \in[0,1]$, se $u$ é uma solução de (3.42), então $u=M(\lambda, u)$, onde

$M(\lambda, u)=Q\left(N_{f}(u)\right)-\frac{B_{\varphi, b}(P u)}{T}+H\left(\varphi^{-1}\left[\varphi(P u)+\lambda H\left(N_{f}(u)-Q\left(N_{f}(u)\right)\right)+\frac{\Psi B_{\varphi, b}(P u)}{T}\right]\right)+P(u)$. 
Avaliando $u$ em 0 , segue que

$$
\int_{0}^{T} f\left(t, u(t), u^{\prime}(t)\right) d t-B_{\varphi, b}(u(0))=0 .
$$

Derivando $u$, obtemos

$$
u^{\prime}(t)=\varphi^{-1}\left[\lambda H\left(N_{f}(u)-Q\left(N_{f}(u)\right)\right)(t)+t \frac{\varphi(b u(0))-\varphi(u(0))}{T}+\varphi(u(0))\right]
$$

para todo $t \in[0, T]$. Avaliando $u^{\prime}$ em 0 , segue que $u^{\prime}(0)=u(0)$. Portanto,

$$
\int_{0}^{T} f\left(t, u(t), u^{\prime}(t)\right) d t-B_{\varphi, b}\left(u^{\prime}(0)\right)=0 .
$$

Usando a hipótese 1 acima, temos

$$
u_{m}^{\prime}<M_{2} \text { e } u_{M}^{\prime}>M_{1} .
$$

Portanto, existe $\omega \in[0, T]$ tal que $M_{1}<u^{\prime}(\omega)<M_{2}$. Além disso,

$$
\int_{\omega}^{t}\left(\varphi\left(u^{\prime}(s)\right)\right)^{\prime} d s=\lambda \int_{\omega}^{t} N_{f}(u)(s) d s+(1-\lambda) \int_{\omega}^{t} Q\left(N_{f}(u)\right)(s) d s
$$

para todo $t \in[0, T]$. Logo, utilizando a condição 2 , segue que

$$
\left|\varphi\left(u^{\prime}(t)\right)\right| \leq\left|\varphi\left(u^{\prime}(\omega)\right)\right|+2\|h\|_{L^{1}}<L+2\|h\|_{L^{1}},
$$

onde $L=\max \left\{\left|\varphi\left(M_{2}\right)\right|,\left|\varphi\left(M_{1}\right)\right|\right\}$. Por conseguinte,

$$
\left\|u^{\prime}\right\|_{\infty}<r_{1}
$$

onde $r_{1}=\max \left\{\left|\varphi^{-1}\left(L+2\|h\|_{L^{1}}\right)\right|,\left|\varphi^{-1}\left(-L-2\|h\|_{L^{1}}\right)\right|\right\}$. Usando o fato de que $u^{\prime}(0)=u(0)$, temos

$$
|u(t)| \leq|u(0)|+\int_{0}^{T}\left|u^{\prime}(s)\right| d t<r_{1}+r_{1} T
$$

para todo $t \in[0, T]$. Desse modo,

$$
\|u\|_{1}=\|u\|_{\infty}+\left\|u^{\prime}\right\|_{\infty}<r_{1}+r_{1} T+r_{1}=r_{1}(2+T)=R_{1} .
$$

Dado $\lambda \in[0,1]$, considere $u \in C^{1}$ tal que $u=Z(\lambda, u)$. Pelo Lema (3.6.4) segue que

$$
\left(\varphi\left(u^{\prime}\right)\right)^{\prime}=\lambda Q\left(N_{f}(u)\right), \quad \int_{0}^{T} f\left(t, u(t), u^{\prime}(t)\right) d t=\varphi(b u(0))-\varphi(u(0)), \quad u^{\prime}(0)=u(0) .
$$

Utilizando novamente a hipótese 1 acima, temos

$$
u_{m}^{\prime}<M_{2} \text { e } u_{M}^{\prime}>M_{1} .
$$

Por conseguinte, existe $\tau \in[0, T]$ tal que $M_{1}<u^{\prime}(\tau)<M_{2}$. Além disso,

$$
\int_{\tau}^{t}\left(\varphi\left(u^{\prime}(s)\right)\right)^{\prime} d s=\lambda \int_{\tau}^{t} Q\left(N_{f}(u)\right)(s) d s
$$

e, portanto, resulta

$$
\left|\varphi\left(u^{\prime}(t)\right)\right| \leq\left|\varphi\left(u^{\prime}(\tau)\right)\right|+\left|\lambda \int_{\tau}^{t} Q\left(N_{f}(u)\right)(s) d s\right|
$$

para todo $t \in[0, T]$. Logo, usando novamente a condição 2 , segue que

$$
\left|\varphi\left(u^{\prime}(t)\right)\right|<L+\|h\|_{L^{1}} .
$$

Por conseguinte,

$$
\left\|u^{\prime}\right\|_{\infty}<r_{2}
$$

onde $r_{2}=\max \left\{\left|\varphi^{-1}\left(L+\|h\|_{L^{1}}\right)\right|,\left|\varphi^{-1}\left(-L-\|h\|_{L^{1}}\right)\right|\right\}$.

Para $t \in[0, T]$ 


$$
|u(t)| \leq|u(0)|+\int_{0}^{T}\left|u^{\prime}(s)\right| d t<r_{2}+r_{2} T
$$

Em consequência,

$$
\|u\|_{1}=\|u\|_{\infty}+\left\|u^{\prime}\right\|_{\infty}<r_{2}+r_{2} T+r_{2}=r_{2}(2+T)=R_{2} .
$$

Definindo $\Omega=B_{\rho}(0)$ no Teorema 3.6.6, onde $\rho$ é um valor real positivo tal que $\rho \geq \max \left\{R_{1}, R_{2}\right\}$, podemos estabelecer a existência de pelo menos uma solução de (3.40).

Teorema 3.6.8. Seja $\varphi$ um homeomorfismo impar. Suponha que as seguintes condições sejam satisfeitas.

1. Existe $h \in C$ tal que

$$
|f(t, x, y)| \leq h(t)
$$

para todo $x, y \in \mathbb{R}$ e todo $t \in[0, T]$.

2. Para um oportuno $r>0$, o grau de Brouwer

$$
\operatorname{deg}_{B}\left(G, B_{r}(0) \cap \mathbb{R}^{2}, 0\right) \neq 0 .
$$

Então, o problema (3.40) com $b=-1$ tem ao menos uma solução.

Demonstração. Para $\lambda \in(0,1]$ e $b=-1$, se $u \in C^{1}$ é uma solução do problema (3.42), então

$$
u=M(\lambda, u),
$$

onde

$M(\lambda, u)=Q\left(N_{f}(u)\right)-\frac{B_{\varphi, b}(P u)}{T}+H\left(\varphi^{-1}\left[\varphi(P u)+\lambda H\left(N_{f}(u)-Q\left(N_{f}(u)\right)\right)+\frac{\Psi B_{\varphi, b}(P u)}{T}\right]\right)+P(u)$.

Como $u^{\prime}(0)$ e $u^{\prime}(T)$ possuem sinais diferentes, existe $\tau \in[0, T]$ tal que $u^{\prime}(\tau)=0$, que implica $\varphi\left(u^{\prime}(\tau)\right)=0 \mathrm{e}$

$$
\begin{aligned}
\varphi\left(u^{\prime}(t)\right) & =\int_{\tau}^{t}\left(\varphi\left(u^{\prime}(s)\right)\right)^{\prime} d s \\
& =\int_{\tau}^{t}\left(\lambda N_{f}(u)+(1-\lambda) Q\left(N_{f}(u)\right)\right)(s) d s .
\end{aligned}
$$

Usando a hipótese 1 acima, temos

$$
\left|\varphi\left(u^{\prime}(t)\right)\right| \leq 2\|h\|_{L^{1}} \quad(t \in[0, T]),
$$

e, portanto,

$$
\left\|u^{\prime}\right\|_{\infty} \leq \mid \varphi^{-1}\left(2\|h\|_{L^{1}} \mid\right) .
$$

Avaliando a função $u$ em 0 e considerando que $\varphi$ é ímpar, temos que

$$
\varphi(u(0))=\frac{-1}{2} \int_{0}^{T} f\left(t, u(t), u^{\prime}(t)\right) d t .
$$

Utilizando novamente a hipótese 1 acima, segue que

$$
|\varphi(u(0))| \leq\|h\|_{L^{1}} .
$$

Portanto,

$$
|u(0)| \leq\left|\varphi^{-1}\left(\|h\|_{L^{1}}\right)\right|
$$

Daí, que para $t \in[0, T]$,

$$
|u(t)| \leq|u(0)|+\int_{0}^{T}\left|u^{\prime}(s)\right| d s \leq\left|\varphi^{-1}\left(\|h\|_{L^{1}}\right)\right|+\mid \varphi^{-1}\left(2\|h\|_{L^{1}} \mid\right) T .
$$


Assim,

$$
\|u\|_{1}=\|u\|_{\infty}+\left\|u^{\prime}\right\|_{\infty} \leq\left|\varphi^{-1}\left(\|h\|_{L^{1}}\right)\right|+\left|\varphi^{-1}\left(2\|h\|_{L^{1}} \mid\right) T+\right| \varphi^{-1}\left(2\|h\|_{L^{1}} \mid\right)=r_{1} .
$$

Seja $\lambda \in[0,1]$. Se $u \in C^{1}$ é tal que $u=Z(\lambda, u)$, então usando o Lema 3.6.4 segue que

$$
\left(\varphi\left(u^{\prime}\right)\right)^{\prime}=\lambda Q\left(N_{f}(u)\right), \quad \int_{0}^{T} f\left(t, u(t), u^{\prime}(t)\right) d t=-2 \varphi(u(0)), \quad u^{\prime}(0)=u(0) .
$$

Portanto,

$$
|\varphi(u(0))| \leq\|h\|_{L^{1}},|u(0)| \leq\left|\varphi^{-1}\left(\|h\|_{L^{1}}\right)\right| .
$$

Como $u=Z(\lambda, u)$, então

$$
\varphi\left(u^{\prime}(t)\right)=\frac{-\lambda t 2 \varphi(u(0))}{T}+\varphi(u(0))
$$

para todo $t \in[0, T]$. Logo,

$$
\left|\varphi\left(u^{\prime}(t)\right)\right| \leq 3|\varphi(u(0))| \leq 3\|h\|_{L^{1}},\left|u^{\prime}(t)\right| \leq\left|\varphi^{-1}\left(3\|h\|_{L^{1}}\right)\right|,
$$

e, portanto,

$$
\left\|u^{\prime}\right\|_{\infty} \leq\left|\varphi^{-1}\left(3\|h\|_{L^{1}}\right)\right|
$$

Assim,

$$
|u(t)| \leq|u(0)|+\int_{0}^{T}\left|u^{\prime}(s)\right| d s \leq\left|\varphi^{-1}\left(\|h\|_{L^{1}}\right)\right|+\left|\varphi^{-1}\left(3\|h\|_{L^{1}}\right)\right| T
$$

para todo $t \in[0, T]$. Logo,

$$
\|u\|_{1}=\|u\|_{\infty}+\left\|u^{\prime}\right\|_{\infty} \leq\left|\varphi^{-1}\left(\|h\|_{L^{1}}\right)\right|+\left|\varphi^{-1}\left(3\|h\|_{L^{1}} \mid\right) T+\right| \varphi^{-1}\left(3\|h\|_{L^{1}} \mid\right)=r_{2} .
$$

Chamando $\Omega=B_{r}(0)$ no Teorema 3.6.6, onde $r$ é um valor real positivo tal que $r>\max \left\{r_{1}, r_{2}\right\}$, podemos garantir a existência de ao menos uma solução de (3.40).

A seguir, o problema (3.40) pode ser estudado aproveitando algumas propriedades particulares do homeomorfismo $\varphi$.

Antes de provarmos o Teorema 3.6.10 que trata da existência de ao menos uma solução do problema (3.40), vamos estudar as seguintes aplicações.

O operador diferencial

$$
D: \operatorname{dom}(D) \subset C_{b}^{1} \longrightarrow C, \quad u \longmapsto u^{\prime},
$$

onde $\operatorname{dom}(D)=\left\{u \in C_{b}^{1}: \varphi\left(u^{\prime}\right) \in C^{1}\right\}, C_{b}^{1}=\left\{u \in C^{1}: u^{\prime}(T)=b u^{\prime}(0), u^{\prime}(0)=u(0)\right\}$.

O operador

$$
D_{\varphi}: \operatorname{dom}\left(D_{\varphi}\right) \longrightarrow C, \quad u \longmapsto(\varphi(u))^{\prime},
$$

onde $\operatorname{dom}\left(D_{\varphi}\right)=\left\{u \in C: \varphi(u) \in C^{1}\right\}$.

O operador

$$
D_{\varphi} D: \operatorname{dom}(D) \longrightarrow C, \quad u \longmapsto\left(\varphi\left(u^{\prime}\right)\right)^{\prime} .
$$

Se $b<0,-\varphi(\cdot)$ e $\varphi(b \cdot)$ são simultaneamente crescentes ou decrescentes. Por conseguinte, $B_{\varphi, b}(\cdot)=\varphi(b \cdot)-\varphi(\cdot)$ é injetora. Assim, o operador $D_{\varphi} D$ tem inverso que é dado por

$$
u \longmapsto H\left(\varphi^{-1}\left[\varphi\left(B_{\varphi, b}^{-1}\left(\int_{0}^{T} u(s) d s\right)\right)+\int_{0}^{t} u(s) d s\right]\right)+B_{\varphi, b}^{-1}\left(\int_{0}^{T} u(s) d s\right) .
$$

Portanto,

$$
\begin{aligned}
& \left(\varphi\left(u^{\prime}\right)\right)^{\prime}=N_{f}(u), u^{\prime}(T)=b u^{\prime}(0), u^{\prime}(0)=u(0) \\
& \Leftrightarrow\left(D_{\varphi} D\right)(u)=N_{f}(u), u \in \operatorname{dom}(D) \\
& \Leftrightarrow u=\left(D_{\varphi} D\right)^{-1} N_{f}(u), u \in C^{1} .
\end{aligned}
$$

Desse modo, nosso objetivo a seguir será encontrar um ponto fixo do operador 


$$
\begin{gathered}
\Gamma:=\left(D_{\varphi} D\right)^{-1} N_{f}: C^{1} \longrightarrow \operatorname{dom}(D) . \\
u \longmapsto H\left(\varphi^{-1}\left[\varphi\left(B_{\varphi, b}^{-1}\left(\int_{0}^{T} N_{f}(u)(s) d s\right)\right)+\int_{0}^{t} N_{f}(u)(s) d s\right]\right)+B_{\varphi, b}^{-1}\left(\int_{0}^{T} N_{f}(u)(s) d s\right) .
\end{gathered}
$$

Lema 3.6.9. O operador $\Gamma$ é completamente contínuo.

Demonstração. Seja $\Lambda$ um conjunto limitado de $C^{1}$. Então, existe $\rho>0$ tal que

$$
\|u\|_{1} \leq \rho
$$

para todo $u \in \Lambda$. Queremos mostrar que $\overline{\Gamma(\Lambda)} \subset \operatorname{dom}(D)$ é um conjunto compacto. Para tanto, tome $\left(v_{n}\right)$ uma sequência em $\Gamma(\Lambda)$ e $\left(u_{n}\right)$ uma sequência em $\Lambda$ tal que $v_{n}=\Gamma\left(u_{n}\right)$. Da fórmula (3.52), segue que existe $W>0$ tal que

$$
\left\|N_{f}\left(u_{n}\right)\right\|_{\infty} \leq W
$$

para todo $n \in \mathbb{N}$. Daí se segue que

$$
\left\|H\left(N_{f}\left(u_{n}\right)\right)\right\|_{\infty} \leq W T .
$$

Portanto, a sequência $\left(H\left(N_{f}\left(u_{n}\right)\right)\right)$ é limitada em $C$. Além disso, para todo $n \in \mathbb{N}$ e para $t, t_{1} \in$ $[0, T]$, segue que

$$
\begin{aligned}
& \left|H\left(N_{f}\left(u_{n}\right)\right)(t)-H\left(N_{f}\left(u_{n}\right)\right)\left(t_{1}\right)\right| \\
& \leq\left|\int_{t_{1}}^{t} N_{f}\left(u_{n}\right)(s) d s\right| \\
& \leq W\left|t-t_{1}\right|
\end{aligned}
$$

o que implica que a sequência $\left(H\left(N_{f}\left(u_{n}\right)\right)\right)$ é equicontínua. Assim, usando o Teorema Arzelà-Ascoli (Teorema 2.3.5), $\left(H\left(N_{f}\left(u_{n}\right)\right)\right)$ possui uma subsequência convergente em $C$, que vamos chamar de

$$
\left(H\left(N_{f}\left(u_{n_{j}}\right)\right)\right) \text {. }
$$

Então, passando a uma subsequência, se necessário, temos que a sequência

$$
\left(\varphi\left(B_{\varphi, b}^{-1}\left(\int_{0}^{T} N_{f}\left(u_{n_{j}}\right)(s) d s\right)\right)+H\left(N_{f}\left(u_{n_{j}}\right)\right)\right)
$$

é convergente em $C$. Sabendo que $\varphi^{-1}: C \longrightarrow C$ é contínua e que

$$
\Gamma\left(u_{n_{j}}\right)^{\prime}(t)=\varphi^{-1}\left[\varphi\left(B_{\varphi, b}^{-1}\left(\int_{0}^{T} N_{f}\left(u_{n_{j}}\right)(s) d s\right)\right)+\int_{0}^{t} N_{f}\left(u_{n_{j}}\right)(s) d s\right]
$$

segue que a sequência $\left(\Gamma\left(u_{n_{j}}\right)^{\prime}\right)$ é convergente em $C$ e, portanto, $\left(v_{n_{j}}\right)=\left(\Gamma\left(u_{n_{j}}\right)\right)$ é convergente em $C^{1}$. Para finalizar a prova, suponha $\left(v_{n}\right) \subseteq \overline{\Gamma(\Lambda)}$. Seja $\left(z_{n}\right) \subseteq \Gamma(\Lambda)$ tal que

$$
\lim _{n \rightarrow \infty}\left\|z_{n}-v_{n}\right\|_{1}=0 .
$$

Seja também $\left(z_{n_{j}}\right)$ uma subsequência de $\left(z_{n}\right)$ que converge para $z$. Segue que $z \in \overline{\Gamma(\Lambda)}$ e $\left(v_{n_{j}}\right)$ converge para $z$. Portanto, o resultado está provado.

Vamos finalmente provar o resultado de existência que generaliza o Teorema 3.6.8.

Teorema 3.6.10. Seja $f$ uma função contínua. Suponha que exista $h \in C$ tal que

$$
|f(t, x, y)| \leq h(t)
$$

para todo $x, y \in \mathbb{R}$ e todo $t \in[0, T]$. Então, o problema (3.40) com $b<0$ tem ao menos uma solução.

Demonstração. Considerando $v=\Gamma(u):=\left(D_{\varphi} D\right)^{-1} N_{f}(u)$, temos,

$$
v^{\prime}(T)=b v^{\prime}(0), v^{\prime}(0)=v(0)
$$

e, também, 


$$
N_{f}(u)=\left(D_{\varphi} D\right)(v)=\left(\varphi\left(v^{\prime}\right)\right)^{\prime} .
$$

Observando que $v^{\prime}(T), v^{\prime}(0)$ possuem sinais diferentes, existe um $\tau \in[0, T], v^{\prime}(\tau)=0$. Portanto, $\varphi\left(v^{\prime}(\tau)\right)=0$. Assim,

$$
\left|\varphi\left(v^{\prime}(t)\right)\right|=\left|\int_{\tau}^{t}\left(\varphi\left(v^{\prime}(s)\right)\right)^{\prime} d s\right| \leq \int_{\tau}^{t}\left|N_{f}(u)(s)\right| d s \leq \int_{0}^{T}\left|f\left(s, u(s), u^{\prime}(s)\right)\right| d s \leq\|h\|_{L^{1}}
$$

para todo $t \in[0, T]$. Logo,

$$
\left\|v^{\prime}\right\|_{\infty} \leq L
$$

onde $L=\max \left\{\left|\varphi^{-1}\left(\|h\|_{L^{1}}\right)\right|,\left|\varphi^{-1}\left(-\|h\|_{L^{1}}\right)\right|\right\}$. Usando o fato de que $v^{\prime}(0)=v(0)$, deduzimos que

$$
|v(t)| \leq|v(0)|+\int_{0}^{t}\left|v^{\prime}(s)\right| d s \leq+|v(0)|+\int_{0}^{T}\left|v^{\prime}(s)\right| d s \leq L+L T \quad(t \in[0, T]),
$$

e, portanto,

$$
\|v\|_{1}=\|v\|_{\infty}+\left\|v^{\prime}\right\|_{\infty} \leq L+L T+L=L(2+T) .
$$

Desta forma, $\Gamma$ é uma aplicação de $C^{1} \mathrm{em} \overline{B_{L(2+T)}(0)}$. Logo, pelo Teorema do ponto fixo de Schauder (Teorema (2.4.10)), $\Gamma$ tem um ponto fixo, ou seja, solução de (3.40).

Provaremos o seguinte resultado que trata da existência de soluções de (3.40) com outras condições sobre $f(t, x, y)$.

Teorema 3.6.11. Consideramos a identificação natural $(a, b) \approx a+b t$ de $\mathbb{R}^{2}$ com as funções afins de $C^{1}$. Suponhamos que $f$ seja uma função continua que satisfaz as seguintes condições para um oportuno $\rho>0$.

1. Existe uma função $c \in C$ tal que

$$
f(t, x, y) \geq c(t)
$$

para todo $(t, x, y) \in[0, T] \times \mathbb{R} \times \mathbb{R}$.

2. Existe $M_{1}<M_{2}$ tal que para toda $u \in C^{1}$,

$$
\int_{0}^{T} f\left(t, u(t), u^{\prime}(t)\right) d t \neq 0 \text { se } u_{m}^{\prime} \geq M_{2},
$$

ou

$$
\int_{0}^{T} f\left(t, u(t), u^{\prime}(t)\right) d t \neq 0 \text { se } u_{M}^{\prime} \leq M_{1} .
$$

3. A equação

$$
G(x, y)=(0,0)
$$

não tem solução em $\partial B_{\rho}(0) \cap \mathbb{R}^{2}$.

4. O grau de Brouwer

$$
\operatorname{deg}_{B}\left(G, B_{\rho}(0) \cap \mathbb{R}^{2}, 0\right) \neq 0 .
$$

Então, o problema (3.40) tem uma solução para $b=1$.

Demonstração. Para $\lambda \in[0,1]$ e $b=1$, temos que o operador ponto fixo associado ao problema (3.42) é dado por

$$
M(\lambda, u)=P(u)+Q\left(N_{f}(u)\right)+H\left(\varphi^{-1}\left[\lambda H\left(N_{f}(u)-Q\left(N_{f}(u)\right)\right)+\varphi(P u)\right]\right) .
$$

Portanto, para $\lambda \in[0,1]$ e $b=1$, se $u$ é solução de (3.42), então

$$
\int_{0}^{T} f\left(t, u(t), u^{\prime}(t)\right) d t=0 .
$$


Desse modo, usando a condição 2 da hipótese, temos

$$
u_{m}^{\prime}<M_{2} \text { e } u_{M}^{\prime}>M_{1},
$$

Por conseguinte, existe um $\omega \in[0, T]$ tal que $M_{1}<u^{\prime}(\omega)<M_{2}$ e

$$
\int_{\omega}^{t}\left(\varphi\left(u^{\prime}(s)\right)\right)^{\prime} d s=\lambda \int_{\omega}^{t} N_{f}(u)(s) d s
$$

para todo $t \in[0, T]$. Logo,

$$
\begin{aligned}
\left|\varphi\left(u^{\prime}(t)\right)\right| & \leq\left|\varphi\left(u^{\prime}(\omega)\right)\right|+\int_{0}^{T}\left|f\left(s, u(s), u^{\prime}(s)\right)\right| d s \\
& \leq\left|\varphi\left(u^{\prime}(\omega)\right)\right|+\int_{0}^{T} f\left(s, u(s), u^{\prime}(s)\right) d s+2 \int_{0}^{T} c^{-}(s) d s \\
& \leq\left|\varphi\left(u^{\prime}(\omega)\right)\right|+2\left\|c^{-}\right\|_{L^{1}} .
\end{aligned}
$$

Assim,

$$
\left|\varphi\left(u^{\prime}(t)\right)\right|<L+2\left\|c^{-}\right\|_{L^{1}},
$$

onde $L=\max \left\{\left|\varphi\left(M_{2}\right)\right|,\left|\varphi\left(M_{1}\right)\right|\right\}$. Portanto,

$$
\left\|u^{\prime}\right\|_{\infty}<r,
$$

onde $r=\max \left\{\left|\varphi^{-1}\left(L+2\left\|c^{-}\right\|_{L^{1}}\right)\right|,\left|\varphi^{-1}\left(-L-2\left\|c^{-}\right\|_{L^{1}}\right)\right|\right\}$.

Como $u$ é tal que $u^{\prime}(0)=u(0)$, então

$$
|u(t)| \leq|u(0)|+\int_{0}^{T}\left|u^{\prime}(s)\right| d s<r+r T
$$

para todo $t \in[0, T]$. Logo,

$$
\|u\|_{1}=\|u\|_{\infty}+\left\|u^{\prime}\right\|_{\infty}<r+r T+r=r(2+T) .
$$

Portanto, (3.42) com $b=1$ não tem solução em $\partial B_{\rho}(0)$ para todo $\lambda \in[0,1]$ e $\rho \geq r(2+T)$.

Sejam $b=1$ e $(\lambda, u) \in[0,1] \times C^{1}$ tal que $u=Z(\lambda, u)$. Pela avaliacão de $u$ em 0 , temos

$$
\int_{0}^{T} f\left(t, u(t), u^{\prime}(t)\right) d t=0 .
$$

Além disso, $u$ é uma função da forma $u(t)=x+y t, y=x$. Assim, por (3.54)

$$
\int_{0}^{T} f(t, x+y t, y) d t=0,
$$

que, juntamente com a hipótese 3 , implica que $u=x+t x \notin \partial B_{\rho}(0)$. Portanto as condições do Teorema 3.6.6 são satisfeitas, a prova está completa.

O seguinte exemplo é uma aplicacão do Teorema 3.6.11.

Exemplo 3.6.12. Vamos considerar o problema

$$
\left(\left(u^{\prime}\right)^{3}\right)^{\prime}=\frac{e^{u^{\prime}}}{2}-1, \quad u(0)=u^{\prime}(0)=u^{\prime}(T) .
$$

Para $M_{1}=-1, M_{2}=1$ e $c(t)=-1$ para todo $t \in[0, T]$. O problema (3.55) tem ao menos uma solucão para $\rho \geq(1+2 T)^{1 / 3}(2+T)$. 


\section{Capítulo 4}

\section{Problemas em dimensão infinita}

O objetivo deste capítulo é o estudo de alguns problemas não lineares de equações diferenciais em espaços de Banach, com condições de contorno. Precisamente, consideramos problemas do tipo

$$
\left\{\begin{array}{l}
\left(\varphi\left(u^{\prime}\right)\right)^{\prime}=f\left(t, u, u^{\prime}\right) \\
l\left(u, u^{\prime}\right)=0 .
\end{array}\right.
$$

Se trata de problemas análogos aos do capítulo anterior só que desta vez são estudados em espaços de Banach. Estamos interessados em determinar condições que garantem existência de soluções dos problemas do tipo acima, com diferentes hipóteses sobre $\varphi$ e $f$ e várias condições de contorno $l\left(u, u^{\prime}\right)=0$. Analogamente ao capítulo anterior, também aqui a abordagem aos problemas é topológica, baseada principalmente no uso dos graus de Leray-Schauder e Nussbaum.

Apresentamos quatro seções. Na primeira introduzimos alguns conceitos e notações básicas. Na segunda seção, estudamos problemas de valor na fronteira do tipo misto com $\varphi: X \longrightarrow X$ um homeomorfismo, $X$ sendo um espaço de Banach. Posteriormente, abordamos problemas com condições de Dirichlet. Finalmente, na última seção provamos a existência de soluções para outros sistemas com condições de contorno do tipo misto. Não encontramos na literatura vários dos resultados obtidos neste capítulo.

\subsection{Notações Básicas}

Usando a notação dada em [19], consideramos $(X,\|\cdot\|)$ o espaço de Banach real e $T$ um valor real positivo fixo. Denotamos a norma usual em $L^{1}=L_{X}^{1}([0, T])$ por $\|\cdot\|_{L^{1}}$. Por $C=C_{X}([0, T])$ indicamos o espaço de Banach das funções contínuas sobre $[0, T]$ com valores em $X$, dotado da norma uniforme $\|\cdot\|_{\infty}$, por $C^{1}=C_{X}^{1}([0, T])$ o espaço de Banach das funções contínuamente diferenciáveis sobre $[0, T]$ com a norma usual $\|u\|_{1}=\max \left\{\|u\|_{\infty},\left\|u^{\prime}\right\|_{\infty}\right\}$. Consideramos também as seguintes aplicações:

o operador de Nemytskii $N_{f}: C^{1} \longrightarrow C$,

$$
N_{f}(u)(t)=f\left(t, u(t), u^{\prime}(t)\right)
$$

o operador integração $H: C \longrightarrow C^{1}$,

$$
H(u)(t)=\int_{0}^{t} u(s) d s
$$

as seguintes aplicações lineares contínuas:

$$
\begin{gathered}
K: C \longrightarrow C^{1}, \quad K(u)(t)=-\int_{t}^{T} u(s) d s, \\
Q: C \longrightarrow C, \quad Q(u)(t)=\frac{1}{T} \int_{0}^{T} u(s) d s \\
S: C \longrightarrow C, \quad S(u)(t)=u(T), \\
P: C \longrightarrow C, \quad P(u)(t)=u(0) .
\end{gathered}
$$




\subsection{Problemas do tipo misto em espaços de Banach}

O objetivo desta seção é estudar a existência de soluções para o seguinte problema não linear:

$$
\left\{\begin{array}{l}
\left(\varphi\left(u^{\prime}\right)\right)^{\prime}=f\left(t, u, u^{\prime}\right) \\
u(T)=0=u^{\prime}(0)
\end{array}\right.
$$

onde $\varphi: X \longrightarrow X$ é um homeomorfismo com inverso Lipschitziano tal que $\varphi(0)=0$ e $f$ : $[0, T] \times X \times X \longrightarrow X$ uma função contínua.

Entendemos por solução do problema (4.1) uma função $u:[0, T] \longrightarrow X$ de classe $C^{1}$ satisfazendo as condições de fronteira e tal que a função $t \longmapsto \varphi\left(u^{\prime}(t)\right)$ seja de classe $C^{1}$ e satisfaça $\left(\varphi\left(u^{\prime}(t)\right)\right)^{\prime}=f\left(t, u(t), u(t)^{\prime}\right)$ para todo $t$ em $[0, T]$.

No artigo [12], o sistema

$$
\left\{\begin{array}{l}
u^{\prime \prime}=f\left(t, u, u^{\prime}\right) \quad 0<t<1 \\
a u(0)-b u^{\prime}(0)=u_{0}, c u(1)+d u^{\prime}(1)=u_{1},
\end{array}\right.
$$

é estudado onde $a, b, c, d \geq 0$ e $a d+b c>0$. É provada a existência de soluções por meio das propriedades da medida de não compacidade de Kuratowski e o Teorema do ponto fixo de Darbo (Teorema 2.5.15).

Em [28] e [40] são usadas as propriedades da medida de não compacidade e o Teorema do ponto fixo de Sadovskii (Teorema 2.5.14) para provar a existência de soluções do seguinte problema:

$$
\left\{\begin{array}{l}
u^{\prime \prime}=f\left(t, u, u^{\prime}\right) \quad a \leq t \leq b \\
c_{1} u(a)-d_{1} u^{\prime}(a)=u_{1}, c_{2} u(b)+d_{2} u^{\prime}(b)=u_{2},
\end{array}\right.
$$

onde $a, b, c_{i}, d_{i} \in \mathbb{R}$ para $i=1,2$ e $f:[0, T] \times X \times X \longrightarrow X$ uma função contínua.

Inspirados nos trabalhos mencionados acima, vamos mostrar a existência de uma solução de (4.1). O procedimento adotado é transformar o sistema (4.1) em um problema de ponto fixo para um operador definido em $C^{1}$. Em seguida, consideramos dois tipos de regularidade para $f$. No caso em que $f$ seja completamente contínua, garantimos a existência de pelo menos uma solução de (4.1) usando a teoria do grau de Leray-Schauder. Enquanto, no caso em que $f$ não seja completamente contínua, impomos condições sobre $f$ em termos da medida de Kuratowski de não compacidade. Usando o grau para aplicações $\alpha$-condensantes (grau de Nussbaum), provamos a existência de uma solução de (4.1). Vamos aqui apresentar esta abordagem.

De forma similar ao capítulo 3 , consideramos a função $\varphi^{-1}$ com abuso de notação como o operador $\varphi^{-1}: C \longrightarrow C$ definido por $\varphi^{-1}(v)(t)=\varphi^{-1}(v(t))$, onde lembramos que $C$ é o espaço de Banach das funções contínuas de $[0, T]$ em $X$ dotado da norma $\|\cdot\|_{\infty}$. É claro que $\varphi^{-1}$ é contínuo e, sendo Lipschitziano, envia conjuntos limitados em conjuntos limitados. Obtemos o seguinte operador definido em $C^{1}$ :

$$
M_{1}(u)=K\left(\varphi^{-1}\left[H\left(N_{f}(u)\right)\right]\right) .
$$

Provamos o lema seguinte.

Lema 4.2.1. Seja $f$ uma função completamente contínua. Então o operador $M_{1}$ é completamente contínuo. Além disso, $u \in C^{1}$ é solução de (4.1) se e somente se $M_{1}(u)=u$.

Demonstração. Seja $\Lambda$ um conjunto limitado de $C^{1}$. Então, existe $\rho>0$ tal que

$$
\|u\|_{1} \leq \rho
$$

para todo $u \in \Lambda$. Queremos mostrar que $\overline{M_{1}(\Lambda)} \subset C^{1}$ é um conjunto compacto. Para tanto, tome $\left(v_{n}\right)$ uma sequência em $M(\Lambda)$ e $\left(u_{n}\right)$ uma sequência em $\Lambda$ tal que $v_{n}=M_{1}\left(u_{n}\right)$. Da fórmula (4.5), segue que existe $L>0$ tal que 


$$
\left\|N_{f}\left(u_{n}\right)\right\|_{\infty} \leq L
$$

para todo $n \in \mathbb{N}$. Daí se segue que

$$
\left\|H\left(N_{f}\left(u_{n}\right)\right)\right\|_{\infty} \leq L T .
$$

Portanto, a sequência $B=\left(H\left(N_{f}\left(u_{n}\right)\right)\right)$ é limitada em $C$. Além disso, para todo $n \in \mathbb{N}$ e para $t, t_{1} \in[0, T]$, segue que

$$
\begin{aligned}
& \left\|H\left(N_{f}\left(u_{n}\right)\right)(t)-H\left(N_{f}\left(u_{n}\right)\right)\left(t_{1}\right)\right\| \\
& =\left\|\int_{0}^{t} f\left(s, u_{n}(s), u_{n}^{\prime}(s)\right) d s-\int_{0}^{t_{1}} f\left(s, u_{n}(s), u_{n}^{\prime}(s)\right) d s\right\| \\
& =\left\|\int_{t_{1}}^{t} f\left(s, u_{n}(s), u_{n}^{\prime}(s)\right) d s\right\| \\
& \leq L\left|t-t_{1}\right|
\end{aligned}
$$

o que implica que a sequência $\left(H\left(N_{f}\left(u_{n}\right)\right)\right)$ é equicontínua.

Por outro lado, para $t \in[0, T]$, denotamos

$$
B(t)=\left\{H\left(N_{f}\left(u_{n}\right)\right)(t): n \in \mathbb{N}\right\}
$$

onde

$$
\begin{aligned}
H\left(N_{f}\left(u_{n}\right)\right)(t) & =\int_{0}^{t} N_{f}\left(u_{n}\right)(s) d s \\
& =\int_{0}^{t} f\left(s, u_{n}(s), u_{n}^{\prime}(s)\right) d s \\
& =t \lim _{m \rightarrow \infty} \sum_{k=1}^{m} f\left(s_{k}, u_{n}\left(s_{k}\right), u_{n}^{\prime}\left(s_{k}\right)\right) \frac{\left(s_{k}-s_{k-1}\right)}{t} .
\end{aligned}
$$

Lembrando que o envoltório convexo de um conjunto $A \subseteq X$ é dado por

$$
\operatorname{conv}(A)=\left\{\sum_{i=1}^{N} \alpha_{i} x_{i}: x_{i} \in A, \alpha_{i} \in \mathbb{R}, \alpha_{i} \geq 0, \sum_{i=1}^{N} \alpha_{i}=1\right\},
$$

segue-se que

$$
\int_{0}^{t} f\left(s, u_{n}(s), u_{n}^{\prime}(s)\right) d s \in t \overline{\operatorname{conv}}\left(\left\{f\left(s, u_{n}(s), u_{n}^{\prime}(s)\right): s \in[0, T], n \in \mathbb{N}\right\}\right),
$$

que implica

$$
B(t)=\left\{H\left(N_{f}\left(u_{n}\right)\right)(t): n \in \mathbb{N}\right\} \subset t \overline{c o n v}\left(\left\{f\left(s, u_{n}(s), u_{n}^{\prime}(s)\right): s \in[0, T], n \in \mathbb{N}\right\}\right) .
$$

Usando o fato de que $f:[0, T] \times X \times X \longrightarrow X$ é completamente contínua, segue que $\alpha(B(t))=0$. Portanto, $B(t)$ é relativamente compacto em $X$. Assim, utilizando o Teorema de Arzelà-Ascoli, $\left(H\left(N_{f}\left(u_{n}\right)\right)\right)$ possui uma subsequência convergente em $C$, que vamos chamar de

$$
\left(H\left(N_{f}\left(u_{n_{j}}\right)\right)\right) \text {. }
$$

Sabendo que $\varphi^{-1}: C \longrightarrow C$ é contínua e que

$$
M_{1}\left(u_{n_{j}}\right)^{\prime}=\varphi^{-1}\left[H\left(N_{f}\left(u_{n_{j}}\right)\right)\right],
$$

segue que a sequência $\left(M_{1}\left(u_{n_{j}}\right)^{\prime}\right)$ é convergente em $C$ e, portanto, $\left(v_{n_{j}}\right)=\left(M_{1}\left(u_{n_{j}}\right)\right)$ é convergente em $C^{1}$. Para finalizar a prova, suponha $\left(v_{n}\right) \subseteq \overline{M_{1}(\Lambda)}$. Seja $\left(z_{n}\right) \subseteq M_{1}(\Lambda)$ tal que

$$
\lim _{n \rightarrow \infty}\left\|z_{n}-v_{n}\right\|_{1}=0
$$

Seja também $\left(z_{n_{j}}\right)$ uma subsequência de $\left(z_{n}\right)$ que converge para $z$. Segue que $z \in \overline{M_{1}(\Lambda)}$ e $\left(v_{n_{j}}\right)$ converge para $z$. Portanto, $M_{1}$ é completamente contínuo. 
Agora vamos provar a segunda parte do lema. Para tanto, se $u$ é uma solução de (4.1), então $u$ satisfaz a equação (4.1), ou seja, para todo $t \in[0, T]$,

$$
\left(\varphi\left(u^{\prime}(t)\right)\right)^{\prime}=N_{f}(u)(t)=f\left(t, u(t), u^{\prime}(t)\right), u(T)=0=u^{\prime}(0) .
$$

Integrando de 0 a $t \in[0, T]$ e usando o fato de que $u^{\prime}(0)=0$, segue que

$$
\varphi\left(u^{\prime}(t)\right)=H\left(N_{f}(u)\right)(t), u(T)=0 .
$$

Compondo com a função $\varphi^{-1}$, obtemos

$$
u^{\prime}=\varphi^{-1}\left(H\left(N_{f}(u)\right)\right), u(T)=0 .
$$

Aplicando o operador $K$ e sendo $u(T)=0$, deduzimos que, se $u$ é solução de (4.1), então

$$
\begin{aligned}
u(t) & =K\left(\varphi^{-1}\left[H\left(N_{f}(u)\right)\right]\right)(t) \\
& =M_{1}(u)(t) .
\end{aligned}
$$

Reciprocamente, se $u \in C^{1}$ é tal que $M_{1}(u)=u$, então para todo $t \in[0, T]$

$$
u(t)=K\left(\varphi^{-1}\left[H\left(N_{f}(u)\right)\right]\right)(t) .
$$

Derivando, obtemos

$$
u^{\prime}(t)=\varphi^{-1}\left[H\left(N_{f}(u)\right)\right](t)
$$

Em particular,

$$
u^{\prime}(0)=0 \quad \text { e } u(T)=0 .
$$

Compondo com a função $\varphi$, segue que

$$
\varphi\left(u^{\prime}(t)\right)=H\left(N_{f}(u)\right)(t), u(T)=0=u^{\prime}(0) .
$$

Agora, derivando novamente, temos que

$$
\left(\varphi\left(u^{\prime}(t)\right)\right)^{\prime}=N_{f}(u)(t), u(T)=0=u^{\prime}(0)
$$

para todo $t \in[0, T]$. Portanto, $u$ é solução de (4.1).

A partir deste ponto, $M_{1}$ será chamado operador ponto fixo associado ao problema (4.1).

Como feito no estudo do capítulo anterior, também aqui introduzimos para $\lambda \in[0,1]$ uma família de problemas de valor na fronteira

$$
\left\{\begin{array}{l}
\left(\varphi\left(u^{\prime}\right)\right)^{\prime}=\lambda f\left(t, u(t), u^{\prime}(t)\right. \\
u(T)=0=u^{\prime}(0) .
\end{array}\right.
$$

Para cada $\lambda \in[0,1]$, definimos o operador ponto fixo $M(\lambda, \cdot)$ sobre $C^{1}$ associado ao sistema (4.7), onde $M$ é definido em $[0,1] \times C^{1}$ por

$$
M(\lambda, u)=K\left(\varphi^{-1}\left[\lambda H\left(N_{f}(u)\right)\right]\right) .
$$

Utilizando um argumento análogo ao Lema 4.2.1, conseguimos mostrar que podemos escrever (4.7) de forma equivalente $M(\lambda, u)=u$ e, também, que $M$ é completamente contínuo.

O seguinte teorema fornece limitações a priori para os possíveis pontos fixos do operador $M$. Além disso, mostra a existência de ao menos uma solução de (4.1).

Teorema 4.2.2. Sejam $f$ uma função completamente contínua e $\varphi^{-1}$ um homeomorfismo Lipschitziano com constante de Lipschitz $k$. Suponha que existam constantes $c_{0}, c_{1} \geq 0$ tais que

$$
\|f(t, x, y)\| \leq c_{0}+c_{1}\|y\| \text {, para todo } x, y \in X \text { e todo } t \in[0, T] .
$$

Então, o problema (4.1) terá pelo menos uma solução. 
Demonstração. Seja $(\lambda, u) \in[0,1] \times C^{1}$ tal que $M(\lambda, u)=u$. Então, usando um argumento análogo ao Lema 4.2.1 temos que $u$ é solução de (4.7), o que implica que

$$
u^{\prime}=\varphi^{-1}\left[\lambda H\left(N_{f}(u)\right)\right], u^{\prime}(0)=0=u(T) .
$$

Usando o fato de que $\varphi^{-1}$ é um homeomorfismo Lipschitziano com constante de Lipschitz $k$, segue-se que

$$
\left\|u^{\prime}(t)\right\| \leq k c_{0} T+k c_{1} \int_{0}^{t}\left\|u^{\prime}(s)\right\| d s
$$

para todo $t \in[0, T]$. Logo, da desigualdade de Gronwall, obtemos

$$
\left\|u^{\prime}(t)\right\| \leq k c_{0} T e^{\int_{0}^{t} k c_{1} d s} \leq k c_{0} T e^{k c_{1} T} .
$$

Portanto, $\left\|u^{\prime}\right\|_{\infty} \leq k c_{0} T e^{k c_{1} T}=: \beta$.

Por outro lado, graças às condições iniciais do problema (4.7), temos que para cada $t \in[0, T]$,

$$
\|u(t)\| \leq \int_{t}^{T}\left\|u^{\prime}(s)\right\| d s \leq \int_{0}^{T}\left\|u^{\prime}(s)\right\| d s \leq \beta T .
$$

Assim,

$$
\|u\|_{1} \leq R_{1} \text {, onde } R_{1}=\max \{\beta, \beta T\} .
$$

Considerando $\rho \in \mathbb{R}$ tal que $\rho>R_{1}$, temos que $\operatorname{deg}_{L S}(I-M(\lambda, \cdot), B(\rho), 0)$ está bem definido. Logo, usando a propriedade de invariância homotópica do grau, podemos afirmar que

$$
\operatorname{deg}_{L S}\left(I-M(0, \cdot), B_{\rho}(0), 0\right)=\operatorname{deg}_{L S}\left(I-M(1, \cdot), B_{\rho}(0), 0\right) .
$$

Por outro lado, $M(0, \cdot)$ é o operador nulo, e por conseguinte,

$$
\operatorname{deg}_{L S}\left(I-M(0, \cdot), B_{\rho}(0), 0\right)=\operatorname{deg}_{L S}\left(I, B_{\rho}(0), 0\right)=1 .
$$

Portanto, existe $u \in B_{\rho}(0)$ tal que $M_{1}(u)=u$, o que significa que $u$ é solução de (4.1).

O problema (4.1) pode ser estudado colocando algumas condições particulares sobre a função $f$ em termos da medida de não compacidade. Provamos o resultado seguinte que generaliza o teorema anterior para quando $T=1$.

Teorema 4.2.3. Seja $\varphi^{-1}$ um homeomorfismo Lipschitziano com constante de Lipschitz $k$. Suponha que as seguintes condições sejam satisfeitas.

1. Existem constantes $c_{0}, c_{1} \geq 0$ tais que

$$
\|f(t, x, y)\| \leq c_{0}+c_{1}\|y\| \text {, para todo } x, y \in X \text { e todo } t \in[0,1] .
$$

2. Para todo $A, B \subset X$ limitados,

$$
\alpha(f([0,1] \times A \times B)) \leq k_{1} \max \{\alpha(A), \alpha(B)\}, \text { onde } 0<k_{1}<1 / 2 k .
$$

Então, o problema (4.1) terá pelo menos uma solução.

Demonstração. Seja $M_{1}$ definido como em (4.4). Dado que $M_{1}$ é uma composição de funções contínuas segue que é contínuo. Além disso, usando a hipótese 1, podemos provar que $M_{1}$ envia conjuntos limitados em conjuntos limitados em $C^{1}$.

Seja $\Lambda$ um conjunto limitado de $C^{1}$. Então, existe $L_{1}>0$ tal que

$$
\left\|N_{f}(u)\right\|_{\infty} \leq L_{1}
$$


para todo $u \in \Lambda$. Por conseguinte, para $t, t_{1} \in[0,1]$, segue que

$$
\begin{aligned}
\left\|\left(M_{1} u\right)^{\prime}(t)-\left(M_{1} u\right)^{\prime}\left(t_{1}\right)\right\| & =\left\|\varphi^{-1}\left[H\left(N_{f}(u)\right)\right](t)-\varphi^{-1}\left[H\left(N_{f}\left(u_{n}\right)\right)\right]\left(t_{1}\right)\right\| \\
& \leq k\left\|H\left(N_{f}(u)\right)(t)-H\left(N_{f}(u)\right)\left(t_{1}\right)\right\| \\
& \leq k\left\|\int_{t_{1}}^{t} f\left(s, u(s), u^{\prime}(s)\right) d s\right\| \\
& \leq k L_{1}\left|t-t_{1}\right|,
\end{aligned}
$$

o que implica que $\left(M_{1} \Lambda\right)^{\prime}$ é equicontínuo. Logo, usando o Lema 2.5.12, temos que existe $\tau \in[0,1]$ ou $\omega \in[0,1] \mathrm{com}$

$$
\alpha_{1}\left(M_{1} \Lambda\right)=\alpha\left(\left(M_{1} \Lambda\right)(\tau)\right)
$$

$\mathrm{Ou}$

$$
\alpha_{1}\left(M_{1} \Lambda\right)=\alpha\left(\left(M_{1} \Lambda\right)^{\prime}(\omega)\right) .
$$

Consideramos o primeiro caso.

$$
\begin{aligned}
\alpha_{1}\left(M_{1} \Lambda\right) & =\alpha\left(\left(M_{1} \Lambda\right)(\tau)\right) \\
& =\alpha\left(\left\{K\left(\varphi^{-1}\left[H\left(N_{f}(u)\right)\right]\right)(\tau): u \in \Lambda\right\}\right) .
\end{aligned}
$$

Pelas propriedades da medida de não compacidade, temos

$$
\begin{aligned}
\alpha_{1}\left(M_{1} \Lambda\right) & \leq \alpha\left(\left\{\int_{\tau}^{1} \varphi^{-1}\left[H\left(N_{f}(u)\right)(s)\right] d s: u \in \Lambda\right\}\right) \\
& \leq(1-\tau) \alpha\left(\overline{\operatorname{conv}}\left\{\varphi^{-1}\left[H\left(N_{f}(u)\right)(s)\right]: s \in[\tau, 1], u \in \Lambda\right\}\right) \\
& \leq \alpha\left(\left\{\varphi^{-1}\left[H\left(N_{f}(u)\right)(s)\right]: s \in[0,1], u \in \Lambda\right\}\right) .
\end{aligned}
$$

Usando o fato de que $\varphi^{-1}$ é $\alpha$-Lipschitz com constante $k$, segue-se que

$$
\begin{aligned}
\alpha_{1}\left(M_{1} \Lambda\right) & \leq k \alpha\left(\left\{H\left(N_{f}(u)\right)(s): s \in[0,1], u \in \Lambda\right\}\right) \\
& \leq k \alpha\left(\left\{\int_{0}^{s} f\left(t, u(t), u^{\prime}(t)\right): s \in[0,1], u \in \Lambda\right\}\right) \\
& \leq k \alpha\left([0,1] \overline{\operatorname{conv}}\left\{f\left(t, u(t), u^{\prime}(t)\right): t \in[0,1], u \in \Lambda\right\}\right) .
\end{aligned}
$$

Aplicando o Lema 2.5.9 e novamente as propriedades da medida de não compacidade, obtemos

$$
\begin{aligned}
\alpha_{1}\left(M_{1} \Lambda\right) & \leq k \alpha\left(\left\{f\left(t, u(t), u^{\prime}(t)\right): t \in[0,1], u \in \Lambda\right\}\right) \\
& \leq k \alpha\left(f\left([0,1] \times \Lambda([0,1]) \times \Lambda^{\prime}([0,1])\right)\right) .
\end{aligned}
$$

Pela condição 2 , temos

$$
\alpha_{1}\left(M_{1} \Lambda\right) \leq k k_{1} \max \left\{\alpha(\Lambda([0,1])), \alpha\left(\Lambda^{\prime}([0,1])\right)\right\} .
$$

Isto implica, pelo Lema 2.5.11, que

$$
\alpha_{1}\left(M_{1} \Lambda\right) \leq 2 k k_{1} \alpha_{1}(\Lambda), \text { onde } 2 k k_{1}<1 .
$$

Consideramos o segundo caso.

$$
\alpha_{1}\left(M_{1} \Lambda\right)=\alpha\left(\left(M_{1} \Lambda\right)^{\prime}(\omega)\right) .
$$

Usando um argumento análogo ao caso anterior, segue-se que

$$
\alpha_{1}\left(M_{1} \Lambda\right) \leq 2 k k_{1} \alpha_{1}(\Lambda) \text {, onde } 2 k k_{1}<1 .
$$

Daí, podemos afirmar que $M_{1}$ é uma $\alpha$-contração.

Definimos a aplicação contínua $\widetilde{M}:[0,1] \times C^{1} \longrightarrow C^{1}$ por 


$$
\widetilde{M}(\lambda, u)=\lambda M_{1}(u) .
$$

Se $(\lambda, u) \in[0,1] \times C^{1}$ é tal que $\widetilde{M}(\lambda, u)=u$, então usando novamente a desigualdade de Gronwall e o fato de que $\varphi$ é um homeomorfismo com inverso Lipschitziano e $\varphi(0)=0$, obtemos a existência de um $r>0$ tal que $\|u\|_{1}<r$.

Para finalizar, vejamos a existência de ao menos uma solução de (4.1). Para isso, usaremos a propriedade de invariância homotópica do grau topológico para aplicações $\alpha$-condensantes.

Seja $B \subset C^{1}$ limitado, então

$$
\begin{aligned}
\alpha_{1}(\widetilde{M}([0,1] \times B)) & =\alpha_{1}(\widetilde{M}(\lambda, u): \lambda \in[0,1], u \in B) \\
& \leq 2 k k_{1} \alpha_{1}(B) .
\end{aligned}
$$

Portanto, para $\lambda \in[0,1]$, o grau $\operatorname{deg}_{N}\left(I-\widetilde{M}(\lambda, \cdot), B_{r}(0), 0\right)$ está bem definido, e a propriedade de invariância homotópica implica que

$$
\operatorname{deg}_{N}\left(I-\widetilde{M}(1, \cdot), B_{r}(0), 0\right)=\operatorname{deg}_{N}\left(I-\widetilde{M}(0, \cdot), B_{r}(0), 0\right) .
$$

Usando o fato de que $\widetilde{M}(0, \cdot)$ é o operador nulo, podemos afirmar que

$$
\operatorname{deg}_{N}(I-\widetilde{M}(0, \cdot), B(\rho), 0)=\operatorname{deg}_{N}\left(I, B_{r}(0), 0\right)=1 .
$$

Então, da propriedade de existência do grau, existe $u \in B_{r}(0)$ tal que $M_{1}(u)=u$, que é uma solução para (4.1).

Observação 4.2.4. Em [12], o termo não linear $f(t, x, y)$ é limitado, enquanto, em nosso resultado, é controlado por um crescimento linear.

Para nossos seguintes resultados $X$ é um espaço de Hilbert real com produto interno $\langle\cdot, \cdot\rangle, \varphi$ é um homeomorfismo tal que $\varphi^{-1}$ é Lipschitziano e $\varphi(0)=0$. Suponhamos que $\varphi$ seja estritamente monótono em $X$ no seguinte sentido:

$$
\langle\varphi(x)-\varphi(y), x-y\rangle>0 \text { para } x \neq y .
$$

No lema seguinte, $M(\lambda, u)$ é o operador definido em (4.8).

Lema 4.2.5. Seja $h \in C\left([0, T], \mathbb{R}^{+}\right)$tal que

$$
\|f(t, x, y)\| \leq\langle f(t, x, y), x\rangle+h(t)
$$

para todo $x, y \in X$ e todo $t \in[0, T]$. Então existem duas constantes $L, R>0$ tais que, se $(\lambda, u) \in$ $[0,1] \times C^{1}$ é tal que $M(\lambda, u)=u$, temos $\left\|u^{\prime}\right\|_{\infty} \leq L$ e $\|u\|_{1} \leq R$.

Demonstração. Seja $(\lambda, u) \in(0,1] \times C^{1}$ tal que $M(\lambda, u)=u$. Então, usando um argumento análogo ao Lema 4.2.1, temos que $u$ é solução de (4.7), o que implica que

$$
\varphi\left(u^{\prime}\right)=\lambda H\left(N_{f}(u)\right), u^{\prime}(0)=0=u(T) .
$$

Portanto, para todo $t \in[0, T]$,

$$
\begin{aligned}
\left\|\lambda H\left(N_{f}(u)\right)(t)\right\| & \leq \int_{0}^{T}\left\|f\left(s, u(s), u^{\prime}(s)\right)\right\| d s \\
& \leq \int_{0}^{T}\left\langle f\left(s, u(s), u^{\prime}(s)\right), u(s)\right\rangle d s+\int_{0}^{T} h(s) d s .
\end{aligned}
$$

Por outro lado, como $\varphi$ é um homeomorfismo tal que

$$
\langle\varphi(y), y\rangle \geq 0
$$

para todo $y \in X$, segue que

$$
\left.\left\langle\varphi\left(u^{\prime}(t)\right)\right), u^{\prime}(t)\right\rangle \geq 0 \quad(t \in[0, T]),
$$


e, por conseguinte,

$$
-\int_{0}^{T}\left\langle\varphi\left(u^{\prime}(t)\right), u^{\prime}(t)\right\rangle d t \leq 0
$$

Usando a integração por partes e as condições iniciais, deduzimos a igualdade

$$
\int_{0}^{T}\left\langle\left(\varphi\left(u^{\prime}(t)\right)\right)^{\prime}, u(t)\right\rangle d t=-\int_{0}^{T}\left\langle\varphi\left(u^{\prime}(t)\right), u^{\prime}(t)\right\rangle d t \leq 0 .
$$

Como $\lambda \in(0,1]$ e $u$ é solução de $(4.7)$, obtemos

$$
\int_{0}^{T}\left\langle f\left(t, u(t), u^{\prime}(t)\right), u(t)\right\rangle d t \leq 0
$$

e, portanto,

$$
\left\|\lambda H\left(N_{f}(u)\right)(t)\right\| \leq\|h\|_{L^{1}}
$$

para todo $t \in[0, T]$. Daí se segue que

$$
\left\|\varphi\left(u^{\prime}(t)\right)\right\| \leq\|h\|_{L^{1}} .
$$

Assim, usando o fato de que $\varphi^{-1}$ é contínua e envia conjuntos limitados em conjuntos limitados, deduzimos que existe um $L>0$ tal que $\left\|u^{\prime}\right\|_{\infty} \leq L$. Utilizando novamente as condições iniciais, temos que para cada $t \in[0, T]$,

$$
\|u(t)\| \leq \int_{t}^{T}\left\|u^{\prime}(s)\right\| d s \leq \int_{0}^{T}\left\|u^{\prime}(s)\right\| d s \leq L T .
$$

Assim,

$$
\|u\|_{1} \leq R, \text { onde } R=\max \{L, L T\} .
$$

Finalmente, se $u=M(0, u)$, então $u=0$, finalizando a demonstração.

O seguinte teorema prova a existência de ao menos uma solução do problema (4.1).

Teorema 4.2.6. Seja $f$ uma função completamente contínua e suponhamos que as condições do Lema 4.2.5 sejam satisfeitas. Então, o problema (4.1) terá pelo menos uma solução.

Demonstração. Usando um argumento análogo ao Lema 4.2.1, temos que $M$ é completamente contínuo. Diante do exposto acima, para cada $\lambda \in[0,1]$ e $\rho>R$, o grau de Leray-Schauder $\operatorname{deg}_{L S}\left(I-M(\lambda, \cdot), B_{\rho}(0), 0\right)$ está bem definido e, pela propriedade de invariância homotópica do grau, temos que

$$
\operatorname{deg}_{L S}\left(I-M(1, \cdot), B_{\rho}(0), 0\right)=\operatorname{deg}_{L S}\left(I-M(0, \cdot), B_{\rho}(0), 0\right) .
$$

Agora, é evidente que o sistema (4.1) é equivalente ao problema

$$
u=M(1, u) .
$$

Por outro lado, observe que $M(0, \cdot)$ é o operador nulo e por conseguinte

$$
\operatorname{deg}_{L S}\left(I-M(0, \cdot), B_{\rho}(0), 0\right)=\operatorname{deg}_{L S}\left(I, B_{\rho}(0), 0\right)=1
$$

Portanto, existe $u \in B_{\rho}(0)$ tal que $u$ é solução de (4.1). $f$.

O teorema seguinte apresenta um resultado de existência com outras condições sobre a função

Teorema 4.2.7. Seja $\varphi^{-1}$ um homeomorfismo com constante de Lipchitz k. Suponhamos que as seguintes condições sejam satisfeitas.

1. A função $f=f(t, x)$ é contínua e envia conjuntos limitados em conjuntos limitados.

2. Existe uma uma função $h \in C\left([0,1], \mathbb{R}^{+}\right)$tal que

$$
\|f(t, x)\| \leq\langle f(t, x), x\rangle+h(t)
$$


para todo $x \in X$ e todo $t \in[0,1]$.

3. Para qualquer subconjunto limitado $S$ de $X$, temos

$$
\alpha(f([0,1] \times S)) \leq k_{1} \alpha(S), \text { para } k k_{1}<1 .
$$

Então, o problema (4.1) terá pelo menos uma solução.

Demonstração. Seja $(\lambda, u) \in[0,1] \times C^{1}$ tal que $M(\lambda, u)=u$. Então pelo Lema 4.2.5, temos a existência de um $R>0$ tal que $\|u\|_{1} \leq R$.

Por outro lado, note que o operador $M$ é contínuo e envia conjuntos limitados em conjuntos limitados. Como $(M([0,1] \times A))^{\prime}$ é equicontínuo para $A \subset C^{1}$ limitado, e usando o Lema 2.5.12, temos que existe $\tau \in[0,1]$ ou $\omega \in[0,1]$ com

$$
\alpha_{1}(M([0,1] \times A))=\alpha(M([0,1] \times A)(\tau))
$$

ou

$$
\alpha_{1}(M([0,1] \times A))=\alpha\left(M([0,1] \times A)^{\prime}(\omega)\right) .
$$

Consideramos o primeiro caso. Usando um argumento análogo ao utilizado no Teorema 4.2.3, temos

$$
\begin{aligned}
\alpha_{1}(M([0,1] \times A)) & \leq k \alpha(\{\lambda f(t, u(t)): \lambda \in[0,1], t \in[0,1], u \in A\}) \\
& \leq k \alpha([0,1] f([0,1] \times A([0,1]))) \\
& \leq k \alpha(f([0,1] \times A([0,1]))) .
\end{aligned}
$$

Aplicando a condição 3 e o Lema 2.5.11, temos

$$
\begin{aligned}
\alpha_{1}(M([0,1] \times A)) & \leq k k_{1} \alpha(A([0,1])) \\
& \leq k k_{1} \alpha_{1}(A),
\end{aligned}
$$

onde $k k_{1}<1$. Consideramos o segundo caso.

$$
\alpha_{1}(M([0,1] \times A))=\alpha\left(M([0,1] \times A)^{\prime}(\omega)\right) .
$$

Usando um argumento análogo ao caso anterior, segue-se que

$$
\alpha_{1}(M([0,1] \times A)) \leq k k_{1} \alpha_{1}(A) \text {, onde } k k_{1}<1 .
$$

Diante do exposto acima, para cada $\lambda \in[0,1]$ e $\rho>R$, o grau de Nussbaum $\operatorname{deg}_{N}\left(I-M(\lambda, \cdot), B_{\rho}(0), 0\right)$ está bem definido e, pela propriedade de invariância homotópica do grau, deduzimos que

$$
\operatorname{deg}_{N}\left(I-M(1, \cdot), B_{\rho}, 0\right)=\operatorname{deg}_{N}\left(I-M(0, \cdot), B_{\rho}, 0\right),
$$

onde

$$
\operatorname{deg}_{N}(I-M(0, \cdot), B(\rho), 0)=\operatorname{deg}_{N}\left(I, B_{\rho}(0), 0\right)=1
$$

Portanto, existe $u \in B_{\rho}(0)$ tal que $u$ é solução de (4.1).

Apresentamos a seguir um teorema de existência para novas condições sobre $f$.

Teorema 4.2.8. Seja $\varphi^{-1}$ um homeomorfismo com constante de Lipchitz $k$. Suponhamos que as seguintes condições sejam satisfeitas.

1. Suponha que para qualquer $R>0$ a função contínua $f=f(t, x)$ seja limitada e uniformemente contínua em $[0,1] \times \overline{B_{R}(0)}$, onde $\overline{B_{R}(0)}=\{x \in X:\|x\| \leq R\}$.

2. Existe uma função $h \in C\left([0,1], \mathbb{R}^{+}\right)$tal que

$$
\|f(t, x)\| \leq\langle f(t, x), x\rangle+h(t)
$$


para todo $x \in X$ e todo $t \in[0,1]$.

3. Existe $0<k_{1}<1 / k$ tal que

$$
\|f(t, x)-f(t, y)\| \leq k_{1}\|x-y\|, \text { para todo } x, y \in X \text { e todo } t \in[0,1] .
$$

Então, o problema (4.1) terá pelo menos uma solução.

Demonstração. Seja $S \subset X$ limitado. Consideremos

$$
H=\left\{\psi_{x}: x \in S\right\}, \text { onde } \psi_{x}(t)=f(t, x) \text { para todo } t \in[0,1] .
$$

É claro que $H$ é um subconjunto limitado em $C$. Como $S$ é limitado em $X$, pela hipotese 1 temos que $H$ é equicontínuo. Portanto, usando o Lema 2.5.8, temos

$$
\alpha_{c}(H)=\alpha(H([0,1]))=\alpha(f([0,1] \times S))=\max _{[0,1]} \alpha(\{f(t, x): x \in S\}) .
$$

Pela condição 3 ,

$$
\alpha(f([0,1] \times S)) \leq k_{1} \alpha(S) .
$$

Portanto, usando o Teorema 4.2.7 garantimos a existência de ao menos uma solução de (4.1).

Consideramos agora o sistema

$$
\left\{\begin{array}{l}
\left(\varphi\left(u^{\prime}\right)\right)^{\prime}=f\left(t, u, u^{\prime}\right) \\
u(0)=0=u^{\prime}(T),
\end{array}\right.
$$

onde $\varphi: X \longrightarrow X$ é um homeomorfismo com inverso Lipschitziano tal que $\varphi(0)=0$ e $f:[0, T] \times$ $X \times X \longrightarrow X$ uma função contínua. O leitor pode observar que temos condições diferentes de contorno, as hipóteses dos teoremas anteriores levam a análogos resultados para o problema (4.10) com o operador ponto fixo associado ao problema (4.10) dado por

$$
M_{2}(u)=H\left(\varphi^{-1}\left[K\left(N_{f}(u)\right)\right]\right) .
$$

Também usando argumentos semelhantes aos estabelecidos nos Teoremas 4.2 .2 e 4.2.3, provamos a existência de pelo menos uma solução dos sistemas

$$
\left\{\begin{array}{l}
\left(\varphi\left(u^{\prime}\right)\right)^{\prime}=f\left(t, u, u^{\prime}\right) \\
u(0)=0=u^{\prime}(0)
\end{array}\right.
$$

e

$$
\left\{\begin{array}{l}
\left(\varphi\left(u^{\prime}\right)\right)^{\prime}=f\left(t, u, u^{\prime}\right) \\
u(T)=0=u^{\prime}(T),
\end{array}\right.
$$

onde os operadores ponto fixo associados aos problemas (4.12) e (4.13) respectivamente são

$$
M_{3}(u)=H\left(\varphi^{-1}\left[H\left(N_{f}(u)\right)\right]\right)
$$

e

$$
M_{4}(u)=K\left(\varphi^{-1}\left[K\left(N_{f}(u)\right)\right]\right) .
$$




\subsection{Problema de Dirichlet em espaços de Banach}

Consideramos o problema de Dirichlet

$$
\left\{\begin{array}{l}
\left(\varphi\left(u^{\prime}\right)\right)^{\prime}=f\left(t, u, u^{\prime}\right) \\
u(0)=0=u(T)
\end{array}\right.
$$

onde $\varphi: X \longrightarrow X$ é um homeomorfismo linear e $f:[0, T] \times X \times X \longrightarrow X$ é uma função contínua.

Uma solução de (4.16) é qualquer função $u:[0, T] \longrightarrow X$ de classe $C^{1}$ que satisfaz as condições de fronteira tal que $t \longmapsto \varphi\left(u^{\prime}(t)\right)$ seja de classe $C^{1}$ e satisfaça $\left(\varphi\left(u^{\prime}(t)\right)\right)^{\prime}=f\left(t, u(t), u(t)^{\prime}\right)$ para todo $t$ em $[0, T]$.

Em [42] Zhou e Peng têm estudado o seguinte sistema:

$$
\left\{\begin{array}{l}
-u^{\prime \prime}=f\left(t, u, u^{\prime}\right) \quad 0 \leq t \leq 1 \\
u(0)=0=u(1)
\end{array}\right.
$$

onde $f:[0,1] \times X \times X \longrightarrow X$ é uma função contínua. Eles conseguem obter a existência de soluções por meio das propriedades da medida de não compacidade e o Teorema do ponto fixo de Sadovskii (Teorema 2.5.14).

Em [26] Mawhin estuda o problema

$$
\left\{\begin{array}{l}
u^{\prime \prime}+k u^{\prime}+f\left(t, u, u^{\prime}\right)=0 \quad 0 \leq t \leq \pi \\
u(0)=0=u(\pi)
\end{array}\right.
$$

onde $f:[0, \pi] \times X \times X \longrightarrow X$ é contínua, $k \in \mathbb{R}$ e $X$ é um espaço de Hilbert real. Ele considera dois tipos de regularidade para a função $f$. No caso em que $f$ seja completamente contínua, Mawhin estabelece a existência de ao menos uma solução de (4.18) usando Teoremas de Leray-Schauder. Para $f$ somente contínua, Mawhin mostra a existência e unicidade de (4.18) sob certas condições relativas à monotonicidade.

Considerando um procedimento análogo ao adotado na seção anterior, mostramos a existência de pelo menos uma solução de (4.16). Transformamos o sistema (4.16) em um problema de ponto fixo para um operador definido em $C^{1}$. Em seguida, consideramos dois tipos de regularidade para $f$. No caso em que $f$ seja completamente contínua, garantimos a existência de pelo menos uma solução usando a teoria do grau de Leray-Schauder. Enquanto, no caso em que $f$ não seja completamente contínua, impomos condições sobre $f$ em termos da medida de Kuratowski de não compacidade. Usando o grau para aplicações $\alpha$-condensantes, provamos a existência de uma solução. Vamos aqui apresentar esta abordagem.

O seguinte lema é uma adaptação de um resultado de [7] para o caso de um homeomorfismo linear definido em um espaço de Banach. o leitor pode ver a analogia com o Lema 3.2.2.

Lema 4.3.1. Para cada $h \in C$, existe um único $Q_{\varphi}=Q_{\varphi}(h) \in X$ tal que

$$
\int_{0}^{T} \varphi^{-1}\left(h(t)-Q_{\varphi}(h)\right) d t=0 .
$$

Além disso, a função $Q_{\varphi}: C \longrightarrow X$ é contínua e envia conjuntos limitados em conjuntos limitados.

Demonstração. Seja $h \in C$ fixada. Definimos a aplicação contínua $G_{h}: X \longrightarrow X$ por

$$
G_{h}(a)=\int_{0}^{T} \varphi^{-1}(h(t)-a) d t .
$$

Vejamos que a equação

$$
G_{h}(a)=0
$$

possui uma única solução $Q_{\varphi}(h)$. Sejam $a, b \in X$ tais que

$$
\int_{0}^{T} \varphi^{-1}(h(t)-a) d t=0, \int_{0}^{T} \varphi^{-1}(h(t)-b) d t=0,
$$


ou seja,

$$
\int_{0}^{T} \varphi^{-1}(h(t)-a) d t=\int_{0}^{T} \varphi^{-1}(h(t)-b) d t .
$$

Usando o fato de que $\varphi^{-1}$ é um homeomorfismo linear, temos $a=b$. Desta forma, se (4.19) tem solução, ela é única. Agora vamos provar a existência. Como $\varphi^{-1}$ é um homeomorfismo linear, então

$$
\int_{0}^{T} \varphi^{-1}(h(t)-a) d t=\varphi^{-1}\left(\int_{0}^{T}(h(t)-a) d t\right) .
$$

Portanto, se $a=\frac{1}{T} \int_{0}^{T} h(t) d t$, então $G_{h}(a)=0$. Por conseguinte, para cada $h \in C$, a equação

$$
G_{h}(a)=0
$$

tem solução. E, como já vimos é única. Desta forma, definimos a aplicação $Q_{\varphi}: C \longrightarrow X$ que satisfaz

$$
\int_{0}^{T} \varphi^{-1}\left(h(t)-Q_{\varphi}(h)\right) d t=0 .
$$

Agora vamos mostrar que $Q_{\varphi}: C \longrightarrow X$ envia conjuntos limitados em conjuntos limitados. Para tanto, seja $A \subset C$ um subconjunto limitado. Então, existe $\rho>0$ tal que

$$
\|h\|_{\infty} \leq \rho
$$

para todo $h \in A$. Por outro lado, como

$$
Q_{\varphi}(A)=\left\{Q_{\varphi}(h): h \in A\right\} \text { e } Q_{\varphi}(h)=\frac{1}{T} \int_{0}^{T} h(t) d t,
$$

então $\|a(h)\| \leq \rho$. Portanto, a função $Q_{\varphi}$ envia conjuntos limitados em conjuntos limitados.

Para finalizar, vamos mostrar que $Q_{\varphi}$ é contínua. Para tanto, seja $\left(h_{n}\right) \subseteq C$ uma sequência tal que

$$
\lim _{n \rightarrow \infty} h_{n}=h \in C .
$$

Como $Q_{\varphi}$ envia conjuntos limitados em conjuntos limitados, então a sequência $\left(Q_{\varphi}\left(h_{n}\right)\right)$ é limitada. Para $n, m \in \mathbb{N}$, temos

$$
\int_{0}^{T} \varphi^{-1}\left(h_{n}(t)-Q_{\varphi}\left(h_{n}\right)\right) d t=\int_{0}^{T} \varphi^{-1}\left(h_{m}(t)-Q_{\varphi}\left(h_{m}\right)\right) d t,
$$

que implica

$$
\left\|\int_{0}^{T}\left(h_{n}(t)-h_{m}(t)\right) d t-\left(Q_{\varphi}\left(h_{n}\right)-Q_{\varphi}\left(h_{m}\right)\right) T\right\|=0 .
$$

Assim,

$$
\left\|Q_{\varphi}\left(h_{n}\right)-Q_{\varphi}\left(h_{m}\right)\right\| T \leq \int_{0}^{T}\left\|\left(h_{n}(t)-h_{m}(t)\right)\right\| d t .
$$

Desse modo, a sequência $\left(Q_{\varphi}\left(h_{n}\right)\right)$ é de Cauchy. Por conseguinte, existe $\widetilde{a} \in X$ tal que

$$
\lim _{n \rightarrow \infty} Q_{\varphi}\left(h_{n}\right)=\widetilde{a} .
$$

Temos, para cada $n \in \mathbb{N}$,

$$
\lim _{n \rightarrow \infty} \int_{0}^{T} \varphi^{-1}\left(h_{n}(t)-Q_{\varphi}\left(h_{n}\right)\right) d t=0 .
$$

Usando o teorema da convergência dominada, deduzimos que

$$
\int_{0}^{T} \varphi^{-1}(h(t)-\widetilde{a}) d t=0 .
$$

Assim, $Q_{\varphi}(h)=\widetilde{a}$, o que mostra que $Q_{\varphi}$ é contínua. 
Utilizando o Lema 4.3.1, definimos o operador $M_{1}: C^{1} \longrightarrow C^{1}$ por

$$
M_{1}(u)=H\left(\varphi^{-1}\left[H\left(N_{f}(u)\right)-Q_{\varphi}\left(H\left(N_{f}(u)\right)\right)\right]\right) .
$$

Da mesma forma que na seção anterior, contemplamos a função $\varphi^{-1}$ com abuso de notação como o operador $\varphi^{-1}: C \longrightarrow C$ definido por $\varphi^{-1}(v)(t)=\varphi^{-1}(v(t))$, onde é claro que $\varphi^{-1}$ é contínuo e, sendo linear, envia conjuntos limitados em conjuntos limitados.

Provamos os seguintes lemas.

Lema 4.3.2. Se $f$ é completamente contínua, então $M_{1}$ é completamente contínuo.

Demonstração. Seja $\Lambda$ um conjunto limitado de $C^{1}$. Então, existe $\rho>0$ tal que

$$
\|u\|_{1} \leq \rho
$$

para todo $u \in \Lambda$. Queremos mostrar que $\overline{M_{1}(\Lambda)} \subset C^{1}$ é um conjunto compacto. Para tanto, tome $\left(v_{n}\right)$ uma sequência em $M(\Lambda)$ e $\left(u_{n}\right)$ uma sequência em $\Lambda$ tal que $v_{n}=M_{1}\left(u_{n}\right)$. Da equação (4.23), segue que existe $L>0$ tal que

$$
\left\|N_{f}\left(u_{n}\right)\right\|_{\infty} \leq L
$$

para todo $n \in \mathbb{N}$. Daí se segue que

$$
\left\|H\left(N_{f}\left(u_{n}\right)\right)\right\|_{\infty} \leq L T .
$$

Portanto, a sequência $B=\left(H\left(N_{f}\left(u_{n}\right)\right)\right)$ é limitada em $C$. Além disso, para todo $n \in \mathbb{N}$ e para $t, t_{1} \in[0, T]$, segue que

$$
\begin{aligned}
& \left\|H\left(N_{f}\left(u_{n}\right)\right)(t)-H\left(N_{f}\left(u_{n}\right)\right)\left(t_{1}\right)\right\| \\
& =\left\|\int_{0}^{t} f\left(s, u_{n}(s), u_{n}^{\prime}(s)\right) d s-\int_{0}^{t_{1}} f\left(s, u_{n}(s), u_{n}^{\prime}(s)\right) d s\right\| \\
& =\left\|\int_{t_{1}}^{t} f\left(s, u_{n}(s), u_{n}^{\prime}(s)\right) d s\right\| \\
& \leq L\left|t-t_{1}\right|
\end{aligned}
$$

o que implica que a sequência $\left(H\left(N_{f}\left(u_{n}\right)\right)\right)$ é equicontínua.

Por outro lado, para $t \in[0, T]$,

$$
B(t)=\left\{H\left(N_{f}\left(u_{n}\right)\right)(t): n \in \mathbb{N}\right\},
$$

onde

$$
\begin{aligned}
H\left(N_{f}\left(u_{n}\right)\right)(t) & =\int_{0}^{t} N_{f}\left(u_{n}\right)(s) d s \\
& =\int_{0}^{t} f\left(s, u_{n}(s), u_{n}^{\prime}(s)\right) d s \\
& =t \lim _{m \rightarrow \infty} \sum_{k=1}^{m} f\left(s_{k}, u_{n}\left(s_{k}\right), u_{n}^{\prime}\left(s_{k}\right)\right) \frac{\left(s_{k}-s_{k-1}\right)}{t} .
\end{aligned}
$$

Daí, segue-se que

$$
\int_{0}^{t} f\left(s, u_{n}(s), u_{n}^{\prime}(s)\right) d s \in t \overline{c o n v}\left(\left\{f\left(s, u_{n}(s), u_{n}^{\prime}(s)\right): s \in[0, T], n \in \mathbb{N}\right\}\right),
$$

que implica

$$
B(t)=\left\{H\left(N_{f}\left(u_{n}\right)\right)(t): n \in \mathbb{N}\right\} \subset t \overline{\operatorname{conv}}\left(\left\{f\left(s, u_{n}(s), u_{n}^{\prime}(s)\right): s \in[0, T], n \in \mathbb{N}\right\}\right) .
$$


Usando a completa continuidade de $f:[0, T] \times X \times X \longrightarrow X$, segue que $\alpha(B(t))=0$. Portanto, $B(t)$ é relativamente compacto em $X$. Assim, usando o Teorema de Arzelà-Ascoli (Teorema 2.3.5), $\left(H\left(N_{f}\left(u_{n}\right)\right)\right)$ possui uma subsequência convergente em $C$, que vamos chamar de

$$
\left(H\left(N_{f}\left(u_{n_{j}}\right)\right)\right) \text {. }
$$

Então, passando a outra subsequência, se necessário, temos que a subsequência

$$
\left(H\left(N_{f}\left(u_{n_{j}}\right)\right)-Q_{\varphi}\left(H\left(N_{f}\left(u_{n_{j}}\right)\right)\right)\right)
$$

é convergente em $C$. Sabendo que $\varphi^{-1}: C \longrightarrow C$ é contínua e que

$$
M_{1}\left(u_{n_{j}}\right)^{\prime}=\varphi^{-1}\left[H\left(N_{f}\left(u_{n_{j}}\right)\right)-Q_{\varphi}\left(H\left(N_{f}\left(u_{n_{j}}\right)\right)\right)\right]
$$

segue que a sequência $\left(M_{1}\left(u_{n_{j}}\right)^{\prime}\right)$ é convergente em $C$ e, portanto, $\left(v_{n_{j}}\right)=\left(M_{1}\left(u_{n_{j}}\right)\right)$ é convergente em $C^{1}$. Para finalizar a prova, suponha $\left(v_{n}\right) \subseteq \overline{M_{1}(\Lambda)}$. Seja $\left(z_{n}\right) \subseteq M_{1}(\Lambda)$ tal que

$$
\lim _{n \rightarrow \infty}\left\|z_{n}-v_{n}\right\|_{1}=0
$$

Seja també $\mathrm{m}\left(z_{n_{j}}\right)$ uma subsequência de $\left(z_{n}\right)$ que converge para $z$. Segue que $z \in \overline{M_{1}(\Lambda)}$ e $\left(v_{n_{j}}\right)$ converge para $z$. Portanto, $M_{1}$ é completamente contínuo.

Lema 4.3.3. $u \in C^{1}$ é solução de (4.16) se, e somente se, é ponto fixo de $M_{1}$.

Demonstração. Se $u$ é uma solução de (4.16), então $u$ satisfaz a equação (4.16), ou seja, para todo $t \in[0, T]$,

$$
\left(\varphi\left(u^{\prime}(t)\right)\right)^{\prime}=N_{f}(u)(t)=f\left(t, u(t), u^{\prime}(t)\right), u(T)=0=u(0) .
$$

Integrando de 0 a $t$, segue que

$$
\varphi\left(u^{\prime}(t)\right)=H\left(N_{f}(u)\right)(t)+a,
$$

onde $a \in X$ é uma constante oportuna. Aplicando a função $\varphi^{-1}$, temos

$$
u^{\prime}(t)=\varphi^{-1}\left[H\left(N_{f}(u)\right)(t)+a\right] .
$$

Integrando novamente de 0 a $t$, deduzimos que

$$
u(t)=u(0)+\int_{0}^{t} \varphi^{-1}\left[H\left(N_{f}(u)\right)(s)+a\right] d s .
$$

Como $u(0)=u(T)=0$, então

$$
\int_{0}^{T} \varphi^{-1}\left[H\left(N_{f}(u)\right)(s)+a\right] d t=0 .
$$

Logo, usando o Lema 4.3.1, temos que $a=-Q_{\varphi}\left(H\left(N_{f}(u)\right)\right)$. Portanto,

$$
\begin{aligned}
u(t) & =\int_{0}^{t} \varphi^{-1}\left[H\left(N_{f}(u)\right)(s)-Q_{\varphi}\left(H\left(N_{f}(u)\right)\right)\right] d s \\
& =M_{1}(u)(t)
\end{aligned}
$$

para todo $t \in[0, T]$.

Reciprocamente, se $u \in C^{1}$ é tal que $M_{1}(u)=u$, então para todo $t \in[0, T]$,

$$
u(t)=H\left(\varphi^{-1}\left[H\left(N_{f}(u)\right)-Q_{\varphi}\left(H\left(N_{f}(u)\right)\right)\right]\right)(t),
$$

onde $u(0)=u(T)=0$. Derivando (4.24), obtemos

$$
u^{\prime}(t)=\varphi^{-1}\left[H\left(N_{f}(u)\right)-Q_{\varphi}\left(H\left(N_{f}(u)\right)\right)\right](t) .
$$


Aplicando a função $\varphi$, segue que

$$
\varphi\left(u^{\prime}(t)\right)=H\left(N_{f}(u)\right)(t)-Q_{\varphi}\left(H\left(N_{f}(u)\right)\right) .
$$

Derivando novamente, temos

$$
\left(\varphi\left(u^{\prime}(t)\right)\right)^{\prime}=N_{f}(u)(t), u(0)=0=u(T)
$$

para todo $t \in[0, T]$. Portanto, $u$ é solução de (4.16).

A partir deste ponto, $M_{1}$ será chamado operador ponto fixo associado ao problema (4.16).

A fim de aplicar o grau de Leray-Schauder a $M_{1}$, introduzimos, para $\lambda \in[0,1]$, a família de problemas de valor na fronteira

$$
\left\{\begin{array}{l}
\left(\varphi\left(u^{\prime}\right)\right)^{\prime}=\lambda f\left(t, u(t), u^{\prime}(t)\right) \\
u(0)=0=u(T) .
\end{array}\right.
$$

Note que (4.25) coincide com (4.16) quando $\lambda=1$. Para cada $\lambda \in[0,1]$, o operador ponto fixo sobre $C^{1}$ associado ao problema (4.25) e introduzido pelo Lema 4.3.3 é $M(\lambda, \cdot)$, onde $M$ é definido em $[0,1] \times C^{1}$ por

$$
M(\lambda, u)=H\left(\varphi^{-1}\left[\lambda H\left(N_{f}(u)\right)-Q_{\varphi}\left(\lambda H\left(N_{f}(u)\right)\right)\right]\right) .
$$

Usando argumentos análogos aos Lemas 4.3.3 e 4.3.2, conseguimos mostrar que podemos escrever o problema (4.25) de forma equivalente $M(\lambda, u)=u$ e, também, que $M$ é completamente contínuo.

A seguir, provamos a existência de ao menos uma solução de (4.16).

Teorema 4.3.4. Seja $f$ uma função completamente continua. Suponha que exista $c>0$ tal que

$$
\|f(t, x, y)\| \leq c, \text { para todo } t \in[0, T] \text { e } x, y \in X .
$$

Então, o problema (4.16) terá pelo menos uma solução.

Demonstração. Seja $(\lambda, u) \in[0,1] \times C^{1}$ tal que $M(\lambda, u)=u$. Então, usando um argumento análogo ao Lema 4.3.3, temos que $u$ é solução de (4.25), o que implica que

$$
u^{\prime}(t)=\varphi^{-1}\left[\lambda H\left(N_{f}(u)\right)(t)-Q_{\varphi}\left(\lambda H\left(N_{f}(u)\right)\right)\right], u(0)=0=u(T) .
$$

Usando o fato de que $\varphi^{-1}$ é um homeomorfismo linear e $f$ uma função limitada, existe $L>0$ tal que

$$
\left\|u^{\prime}(t)\right\| \leq L
$$

para todo $t \in[0, T]$ e para todo $\lambda \in[0,1]$ (lembrando que $u$ é associada a $\lambda$ ).

Por outro lado, graças às condições iniciais do problema (4.25), temos que para cada $t \in[0, T]$,

$$
\|u(t)\| \leq \int_{0}^{t}\left\|u^{\prime}(s)\right\| d s \leq \int_{0}^{T}\left\|u^{\prime}(s)\right\| d s \leq L T .
$$

Assim,

$$
\|u\|_{1} \leq R, \text { onde } R=\max \{L, L T\} .
$$

Tomando $\rho>R$, temos que, para cada $\lambda \in[0,1], \operatorname{deg}_{L S}(I-M(\lambda, \cdot), B(\rho), 0)$ está bem definido e, pela propriedade de invariância homotópica do grau, podemos deduzir que

$$
\operatorname{deg}_{L S}\left(I-M(0, \cdot), B_{\rho}(0), 0\right)=\operatorname{deg}_{L S}\left(I-M(1, \cdot), B_{\rho}(0), 0\right),
$$

onde

$$
\operatorname{deg}_{L S}\left(I-M(0, \cdot), B_{\rho}(0), 0\right)=\operatorname{deg}_{L S}\left(I, B_{\rho}(0), 0\right)=1
$$

Portanto, existe $u \in B_{\rho}(0)$ tal que é solução de (4.16). 
O problema (4.16) pode ser estudado colocando algumas condições particulares sobre a função $f$ em termos da medida de não compacidade. Provamos o resultado seguinte que generaliza o teorema anterior.

Teorema 4.3.5. Seja $\varphi^{-1}$ um homeomorfismo linear com norma $k$. Suponha que as seguintes condições são satisfeitas:

1. Existe um $c>0$ tal que

$$
\|f(t, x, y)\| \leq c, \text { para todo } t \in[0,1] \text { e } x, y \in X .
$$

2. Para todo $A, B \subset X$ limitados

$$
\alpha(f([0,1] \times A \times B)) \leq k_{1} \max \{\alpha(A), \alpha(B)\}, \text { onde } 0<k_{1}<1 / 4 k .
$$

Então, o problema (4.16) terá pelo menos uma solução.

Demonstração. Como $M_{1}$ é uma composição de funções contínuas, segue que $M_{1}$ é contínuo. Além disso, usando a hipótese 1 , temos que $M_{1}$ envia conjuntos limitados em conjuntos limitados em $C^{1}$.

Seja $\Lambda$ um conjunto limitado de $C^{1}$. Então, se $u \in \Lambda$, existe $L>0$ tal que

$$
\left\|N_{f}(u)\right\|_{\infty} \leq L .
$$

Por conseguinte, para $t, t_{1} \in[0,1]$, segue que

$$
\begin{aligned}
\left\|M_{1}(u)^{\prime}(t)-M_{1}(u)^{\prime}\left(t_{1}\right)\right\| & =\left\|\varphi^{-1}\left[H\left(N_{f}(u)\right)\right](t)-\varphi^{-1}\left[H\left(N_{f}(u)\right)\right]\left(t_{1}\right)\right\| \\
& \leq k\left\|H\left(N_{f}(u)\right)(t)-H\left(N_{f}(u)\right)\left(t_{1}\right)\right\| \\
& =k\left\|\int_{0}^{t} f\left(s, u(s), u^{\prime}(s)\right) d s-\int_{0}^{t_{1}} f\left(s, u(s), u^{\prime}(s)\right) d s\right\| \\
& =k\left\|\int_{t_{1}}^{t} f\left(s, u(s), u^{\prime}(s)\right) d s\right\| \\
& \leq k L\left|t-t_{1}\right|
\end{aligned}
$$

o que implica que a $\left(M_{1} \Lambda\right)^{\prime}$ é equicontínuo. Logo, usando o Lema 2.5.12, temos que existe $\tau \in[0,1]$ ou $\omega \in[0,1] \mathrm{com}$

$$
\alpha_{1}\left(M_{1} \Lambda\right)=\alpha\left(\left(M_{1} \Lambda\right)(\tau)\right)
$$

$\mathrm{Ou}$

$$
\alpha_{1}\left(M_{1} \Lambda\right)=\alpha\left(\left(M_{1} \Lambda\right)^{\prime}(\omega)\right)
$$

Consideramos o primeiro caso.

$$
\begin{aligned}
\alpha_{1}\left(M_{1} \Lambda\right) & =\alpha\left(\left(M_{1} \Lambda\right)(\tau)\right) \\
& =\alpha\left(\left\{H\left(\varphi^{-1}\left[H\left(N_{f}(u)\right)-Q_{\varphi}\left(H\left(N_{f}(u)\right)\right)\right]\right)(\tau): u \in \Lambda\right\}\right) .
\end{aligned}
$$

Pelas propriedades da medida de não compacidade, temos

$$
\begin{aligned}
\alpha_{1}\left(M_{1} \Lambda\right) & \leq \alpha\left(\left\{\int_{0}^{\tau} \varphi^{-1}\left[H\left(N_{f}(u)\right)(s)-Q_{\varphi}\left(H\left(N_{f}(u)\right)\right)\right] d s: u \in \Lambda\right\}\right) \\
& \leq \tau \alpha\left(\overline{c o n v}\left\{\varphi^{-1}\left[H\left(N_{f}(u)\right)(s)-Q_{\varphi}\left(H\left(N_{f}(u)\right)\right)\right]: s \in[0, \tau], u \in \Lambda\right\}\right) \\
& \leq \alpha\left(\left\{\varphi^{-1}\left[H\left(N_{f}(u)\right)(s)-Q_{\varphi}\left(H\left(N_{f}(u)\right)\right)\right]: s \in[0,1], u \in \Lambda\right\}\right) .
\end{aligned}
$$


Usando o fato de que $\varphi^{-1}$ é um homeomorfismo linear com norma $k$, segue-se que

$$
\begin{aligned}
\alpha_{1}\left(M_{1} \Lambda\right) & \leq k \alpha\left(\left\{H\left(N_{f}(u)\right)(s)-Q_{\varphi}\left(H\left(N_{f}(u)\right)\right): s \in[0,1], u \in \Lambda\right\}\right) \\
& \leq k \alpha\left(\left\{\int_{0}^{s} f\left(t, u(t), u^{\prime}(t)\right): s \in[0,1], u \in \Lambda\right\}\right)+k \alpha\left(\left\{Q_{\varphi}\left(H\left(N_{f}(u)\right)\right): u \in \Lambda\right\}\right) .
\end{aligned}
$$

Por outro lado, como $Q_{\varphi}\left(H\left(N_{f}(u)\right)\right)=\int_{0}^{1} H\left(N_{f}(u)\right)(s)$, então

$$
\begin{aligned}
\alpha\left(\left\{Q_{\varphi}\left(H\left(N_{f}(u)\right)\right): u \in \Lambda\right\}\right) & \leq \alpha\left(\left\{\int_{0}^{1} H\left(N_{f}(u)\right)(s): u \in \Lambda\right\}\right) \\
& \leq \alpha\left(\left\{\int_{0}^{s} f\left(t, u(t), u^{\prime}(t)\right): s \in[0,1], u \in \Lambda\right\}\right) .
\end{aligned}
$$

Portanto,

$$
\begin{aligned}
\alpha_{1}\left(M_{1} \Lambda\right) & \leq 2 k \alpha\left(\left\{\int_{0}^{s} f\left(t, u(t), u^{\prime}(t)\right): s \in[0,1], u \in \Lambda\right\}\right) \\
& \leq 2 k \alpha\left([0,1] \overline{\operatorname{conv}}\left\{f\left(t, u(t), u^{\prime}(t)\right): t \in[0,1], u \in \Lambda\right\}\right) .
\end{aligned}
$$

Aplicando o Lema 2.5.9 e novamente as propriedades da medida de não compacidade, obtemos

$$
\begin{aligned}
\alpha_{1}\left(M_{1} \Lambda\right) & \leq 2 k \alpha\left(\left\{f\left(t, u(t), u^{\prime}(t)\right): t \in[0,1], u \in \Lambda\right\}\right) \\
& \leq 2 k \alpha\left(f\left([0,1] \times \Lambda([0,1]) \times \Lambda^{\prime}([0,1])\right)\right) .
\end{aligned}
$$

Pela condição 2, temos

$$
\alpha_{1}\left(M_{1} \Lambda\right) \leq 2 k k_{1} \max \left\{\alpha(\Lambda([0,1])), \alpha\left(A^{\prime}([0,1])\right)\right\} .
$$

Isto implica, pelo Lema 2.5.11, que

$$
\alpha_{1}\left(M_{1} \Lambda\right) \leq 4 k k_{1} \alpha_{1}(\Lambda), \text { onde } 4 k k_{1}<1 .
$$

Consideramos o segundo caso.

$$
\alpha_{1}\left(M_{1} \Lambda\right)=\alpha\left(\left(M_{1} \Lambda\right)^{\prime}(\omega)\right) .
$$

Usando um argumento análogo ao caso anterior, segue-se que

$$
\alpha_{1}\left(M_{1} \Lambda\right) \leq 4 k k_{1} \alpha_{1}(\Lambda), \text { onde } 4 k k_{1}<1 .
$$

Daí, podemos afirmar que $M_{1}$ é uma $\alpha$-contração.

Para finalizar, vamos mostrar a existência de uma solução de (4.16). Para tanto, seja $v=M_{1}(u)$ para $u \in C^{1}$. Então para todo $t \in[0,1]$,

$$
v^{\prime}(t)=\varphi^{-1}\left[H\left(N_{f}(u)\right)(t)-Q_{\varphi}\left(H\left(N_{f}(u)\right)\right)\right] .
$$

Usando o fato de que $\varphi^{-1}$ é um homeomorfismo linear e $f$ é limitada, existe $\rho>0$ tal que

$$
\left\|v^{\prime}(t)\right\| \leq \rho
$$

para todo $t \in[0,1]$. Como $v(0)=v(1)=0$, então

$$
\|v(t)\| \leq \int_{0}^{t}\left\|v^{\prime}(s)\right\| d s \leq \int_{0}^{1}\left\|v^{\prime}(s)\right\| d s \leq \rho \quad(t \in[0,1]) .
$$

Assim,

$$
\|v\|_{1} \leq \rho
$$

Desta forma $M_{1}$ é uma aplicação de $C^{1}$ em $\overline{B_{\rho}(0)}$. Logo, pelo Teorema do ponto fixo de Darbo (Teorema 2.5.15), $M_{1}$ tem um ponto fixo, ou seja, solução de (4.16). 
Para nossos seguintes resultados, $X$ é um espaço de Hilbert real com produto interno $\langle\cdot, \cdot\rangle, \varphi$ é um homeomorfismo linear. Suponhamos que $\varphi$ seja estritamente monótono em $X$ no seguinte sentido:

$$
\langle\varphi(x), x\rangle>0 \text { para } x \neq 0 .
$$

Usando argumentos análogos aos do problema (4.1), provamos a existência de ao menos uma solução de (4.16). As demonstrações podem ser omitidas devido à semelhança com as provas conseguidas para (4.1).

Lema 4.3.6. Seja $h \in C\left([0, T], \mathbb{R}^{+}\right)$tal que

$$
\|f(t, x, y)\| \leq\langle f(t, x, y), x\rangle+h(t)
$$

para todo $x, y \in X$ e todo $t \in[0, T]$. Então existem duas constantes $L_{1}, R_{1}>0$ tais que, se $(\lambda, u) \in[0,1] \times C^{1}$ é tal que $M(\lambda, u)=u$, temos $\left\|u^{\prime}\right\|_{\infty} \leq L_{1}$ e $\|u\|_{1} \leq R_{1}$.

Teorema 4.3.7. Seja $f$ uma função completamente contínua e suponhamos que as condições do Lema 4.3.6 sejam satisfeitas. Então, o problema (4.16) terá pelo menos uma solução.

Teorema 4.3.8. Seja $\varphi^{-1}$ um homeomorfismo com norma $k$. Suponhamos que as seguintes condições sejam satisfeitas.

1. A função $f=f(t, x)$ é contínua e envia conjuntos limitados em conjuntos limitados.

2. Existe uma uma função $h \in C\left([0,1], \mathbb{R}^{+}\right)$tal que

$$
\|f(t, x)\| \leq\langle f(t, x), x\rangle+h(t)
$$

para todo $x \in X$ e todo $t \in[0,1]$.

3. Para qualquer subconjunto limitado $S$ de $X$, temos

$$
\alpha(f([0,1] \times S)) \leq k_{1} \alpha(S), \text { para } 2 k k_{1}<1 .
$$

Então, o problema (4.16) terá pelo menos uma solução.

Teorema 4.3.9. Seja $\varphi^{-1}$ um homeomorfismo com norma $k$. Suponhamos que as seguintes condições sejam satisfeitas.

1. Suponha que para qualquer $R>0$ a função contínua $f=f(t, x)$ seja limitada e uniformemente continua em $[0,1] \times \overline{B_{R}(0)}$, onde $\overline{B_{R}(0)}=\{x \in X:\|x\| \leq R\}$.

2. Existe uma uma função $h \in C\left([0,1], \mathbb{R}^{+}\right)$tal que

$$
\|f(t, x)\| \leq\langle f(t, x), x\rangle+h(t)
$$

para todo $x \in X$ e todo $t \in[0,1]$.

3. Existe $0<k_{1}<1 / 2 k$ tal que

$$
\|f(t, x)-f(t, y)\| \leq k_{1}\|x-y\|, \text { para todo } x, y \in X \text { e todo } t \in[0,1] \text {. }
$$

Então, o problema (4.16) terá pelo menos uma solução. 


\subsection{Problemas de tipo misto com outras condições de fronteira}

Consideramos o seguinte sistema não linear:

$$
\left\{\begin{array}{l}
\left(\varphi\left(u^{\prime}\right)\right)^{\prime}=f\left(t, u, u^{\prime}\right) \\
u(0)=u(T)=u^{\prime}(T),
\end{array}\right.
$$

onde $\varphi: X \longrightarrow X$ é um homeomorfismo linear e $f:[0, T] \times X \times X \longrightarrow X$ é uma função contínua.

Entendemos por solução do problema (4.27) uma função $u:[0, T] \longrightarrow X$ de classe $C^{1}$ satisfazendo as condições de fronteira e tal que a função $t \longmapsto \varphi\left(u^{\prime}(t)\right)$ seja de classe $C^{1}$ e satisfaça $\left(\varphi\left(u^{\prime}(t)\right)\right)^{\prime}=f\left(t, u(t), u(t)^{\prime}\right)$ para todo $t$ em $[0, T]$.

Inspirados nos trabalhos $[42,12,28,40,26]$, mostramos a existência de uma solução de (4.27). Por procedimento inteiramente análogo ao adotado no problema em dimensão finita (3.8), transformamos o sistema (4.27) em um problema equivalente de ponto fixo. Em seguida, consideramos dois tipos de regularidade para $f$. No caso em que $f$ seja completamente contínua e limitada, garantimos a existência de pelo menos uma solução de (4.27) usando o Teorema do ponto fixo de Schauder (Teorema 2.4.10). Enquanto, no caso em que $f$ não seja completamente contínua, impomos condições sobre $f$ em termos da medida de Kuratowski de não compacidade. Usando o Teorema do ponto fixo de Darbo (Teorema 2.5.15), provamos a existência de uma solução de (4.27). Vamos aqui apresentar esta abordagem.

Pelo Teorema 3.3.1, podemos escrever o problema (4.27) da seguinte forma equivalente

$$
u=M_{1}(u),
$$

onde

$$
M_{1}(u)=\varphi^{-1}\left(-Q_{\varphi}\left(K\left(N_{f}(u)\right)\right)\right)+H\left(\varphi^{-1}\left[K\left(N_{f}(u)\right)-Q_{\varphi}\left(K\left(N_{f}(u)\right)\right)\right]\right) .
$$

Utilizando um argumento semelhante ao do Lema 4.3.2, podemos mostrar que se $f$ é completamente contínua, então $M_{1}$ é completamente contínuo.

Provamos o seguinte resultado.

Teorema 4.4.1. Seja $f$ uma função completamente continua. Suponha que exista $c>0$ tal que

$$
\|f(t, x, y)\| \leq c, \text { para todo } t \in[0, T] \text { e } x, y \in X .
$$

Então, o problema (4.27) terá pelo menos uma solução.

Demonstração. Seja $v=M_{1}(u)$ para $u \in C^{1}$. Então para todo $t \in[0,1]$,

$$
v(t)=\varphi^{-1}\left(-Q_{\varphi}\left(K\left(N_{f}(u)\right)\right)\right)+H\left(\varphi^{-1}\left[K\left(N_{f}(u)\right)-Q_{\varphi}\left(K\left(N_{f}(u)\right)\right)\right]\right)(t) .
$$

Assim,

$$
\|v(t)\| \leq\left\|\varphi^{-1}\right\|\left(\left\|Q_{\varphi}\left(K\left(N_{f}(u)\right)\right)\right\|+\left\|K\left(N_{f}(u)\right)\right\|_{\infty} T+\left\|Q_{\varphi}\left(K\left(N_{f}(u)\right)\right)\right\| T\right) .
$$

Como $Q_{\varphi}\left(K\left(N_{f}(u)\right)\right)=\frac{1}{T} \int_{0}^{T} K\left(N_{f}(u)\right)(s) d s$ e $\left\|K\left(N_{f}(u)\right)\right\|_{\infty} \leq c T$, então

$$
\|v(t)\| \leq\left\|\varphi^{-1}\right\|\left(c T+2 c T^{2}\right)
$$

para todo $t \in[0, T]$.

Por outro lado, de (4.28), obtemos

$$
\left\|v^{\prime}(t)\right\| \leq 2 c T\left\|\varphi^{-1}\right\|
$$

para todo $t \in[0, T]$. Assim, deduzimos que existe um $r>0$ tal que

$$
\|v\|_{1} \leq r .
$$

Desta forma $M_{1}$ é uma aplicação de $C^{1} \mathrm{em} \overline{B_{r}(0)}$. Logo, pelo Teorema do ponto fixo de Schauder (Teorema 2.4.10), $M_{1}$ tem um ponto fixo, ou seja, solução de (4.27). 
Nosso próximo resultado generaliza o teorema anterior.

Teorema 4.4.2. Seja $\varphi^{-1}$ um homeomorfismo linear com norma $k$. Suponha que as seguintes condições são satisfeitas:

1. Existe $c>0$ tal que

$$
\|f(t, x, y)\| \leq c, \text { para todo } t \in[0,1] \text { e } x, y \in X
$$

2. Para todo $A, B \subset X$ limitados

$$
\alpha(f([0,1] \times A \times B)) \leq k_{1} \max \{\alpha(A), \alpha(B)\}, \text { onde } 0<k_{1}<1 / 6 k .
$$

Então, o problema (4.27) terá pelo menos uma solução.

Demonstração. Por um argumento semelhante ao do Lema 4.3.5, podemos demonstrar que

$$
\alpha_{1}\left(M_{1} \Lambda\right) \leq 6 k k_{1} \alpha_{1}(\Lambda), \text { onde } 6 k k_{1}<1
$$

para todo conjunto $\Lambda$ limitado de $C^{1}$. Por conseguinte, $M_{1}$ é uma $\alpha$-contração. Logo, pelo Teorema do ponto fixo de Darbo (Teorema 2.5.15), $M_{1}$ tem um ponto fixo, ou seja, solução de (4.27).

Teorema 4.4.3. Seja $\varphi^{-1}$ um homeomorfismo com norma $k$. Suponhamos que as seguintes condições sejam satisfeitas.

1. Existe $c>0$ tal que

$$
\|f(t, x)\| \leq c, \text { para todo } x \in X \text { e todo } t \in[0,1] .
$$

2. Existe $0<k_{1}<1 / 3 k$ tal que

$$
\|f(t, x)-f(t, y)\| \leq k_{1}\|x-y\|, \quad \text { para todo } x, y \in X \text { e todo } t \in[0,1] .
$$

3. Para cada $x \in X, D_{1} f(t, x)$ a derivada parcial existe para todo $t \in[0,1]-\Gamma$, onde $\Gamma$ é um subconjunto enumerável em $[0,1]$.

4. Existe $L \geq 0$ tal que

$$
\left\|D_{1} f(t, x)\right\| \leq L, \text { para todo } x \in X \text { e todo } t \in[0,1]-\Gamma .
$$

Então, o problema (4.27) terá pelo menos uma solução.

Demonstração. Seja $S \subset X$ limitado. Consideremos

$$
H=\left\{\psi_{x}: x \in S\right\}, \text { onde } \psi_{x}(t)=f(t, x) \text { para todo } t \in[0,1] .
$$

É claro que $H$ é um subconjunto limitado em $C$. Agora vejamos que $H$ é equicontínuo. Para tanto, seja $x \in S$ e $t, \tau \in[0,1]$. Usando o Teorema do Valor Médio 2.3.7, temos

$$
\left\|\psi_{x}(t)-\psi_{x}(\tau)\right\|=\|f(t, x)-f(\tau, x)\| \leq L|t-\tau| .
$$

Portanto, $H$ é limitado e equicontínuo. Logo, usando o Lema 2.5.8, obtemos

$$
\alpha(H)=\alpha(H([0,1]))=\alpha(f([0,1] \times S))=\max _{[0,1]} \alpha(\{f(t, x): x \in S\}) .
$$

Pela condição 2 ,

$$
\alpha(f([0,1] \times S)) \leq k_{1} \alpha(S) .
$$

Portanto, usando o Teorema 4.4.2 (para $f=f(t, x)$ ), garantimos a existência de ao menos uma solução de (4.27). 


\section{Capítulo 5}

\section{Problemas abertos}

Considerando sistemas do tipo

$$
\left\{\begin{array}{l}
\left(\varphi\left(u^{\prime}\right)\right)^{\prime}=f\left(t, u, u^{\prime}\right) \\
l\left(u, u^{\prime}\right)=0
\end{array}\right.
$$

seja em dimensão finita, seja infinita, dependendo das hipóteses sobre $f$ e $\varphi$ e das condições de contorno, muitos problemas estão ainda em aberto. A seguir, apresentamos alguns deles que estão sendo estudados atualmente.

\section{PROBLEMA 1:}

$$
\left\{\begin{array}{l}
\left(\varphi\left(u^{\prime}\right)\right)^{\prime}=f\left(t, u, u^{\prime}\right) \\
u(0)=u(T)=u^{\prime}(T)
\end{array}\right.
$$

onde

- $f:[0, T] \times X \times X \rightarrow X$ e $X$ um espaço de Banach real.

- $\varphi: X \rightarrow X$ é um homeomorfismo com $\varphi(0)=0$.

No caso em que $\varphi$ seja um homeomorfismo linear e $f$ seja completamente contínua e limitada, no capítulo anterior foi mostrada a existência de ao menos uma solução usando a teoria do grau de Leray-Schauder. Se $f$ não é completamente contínua, impomos condições sobre $f$ em termos da medida de Kuratowski de não compacidade. Usando o grau para aplicações $\alpha$-condensantes, garantimos a existência de ao menos uma solução. Atualmente procuramos estender o resultado para quando $f$ não seja limitada e $\varphi$ não seja linear. Tal problema não parece ter sido considerado na literatura.

\section{PROBLEMA 2:}

$$
\left\{\begin{array}{l}
\left(\varphi\left(u^{\prime}\right)\right)^{\prime}=f\left(t, u, u^{\prime}\right) \\
u(0)=0=u(T)
\end{array}\right.
$$

onde

- $f:[0, T] \times \mathbb{R} \times \mathbb{R} \rightarrow \mathbb{R}$ é uma função contínua.

- $\varphi: \mathbb{R} \rightarrow(-a, a)$ é um homeomorfismo com $\varphi(0)=0$.

O objetivo é estudar a existência de soluções do problema para valores na fronteira do tipo Dirichlet no caso em que a função $f$ só é limitada por cima ou por baixo por uma função contínua. O problema foi apresentado por Bereanu e Mawhin em [8] e atualmente permanece aberto. 
No entanto, no caso em que $f$ seja limitada, conseguimos mostrar a existência de ao menos uma solução (Ver Teorema 3.2.9).

PROBLEMA 3:

$$
\left\{\begin{array}{l}
\left(\varphi\left(u^{\prime}\right)\right)^{\prime}=f\left(t, u, u^{\prime}\right) \\
u(0)=u^{\prime}(0), \quad u^{\prime}(T)=b u^{\prime}(0),
\end{array}\right.
$$

onde

- $b$ é um número real diferente de zero.

- $f:[0, T] \times \mathbb{R} \times \mathbb{R} \rightarrow \mathbb{R}$ é uma função contínua.

- $\varphi: \mathbb{R} \rightarrow(-a, a)$ é um homeomorfismo com $\varphi(0)=0$.

O problema surgiu como consequência dessa pesquisa. Atualmente conhecemos resultados de existência para quando $\varphi$ é do tipo clássico. Veja Teoremas 3.6.6 e 3.6.10. 


\section{Referências Bibliográficas}

[1] H. Amann and S.A. Weiss, On the uniqueness of the topological degree, Math. Z. 130 (1973), 39-54. 13

[2] P. Benevieri, J. M. do Ó, E. Souto de Medeiros, Periodic solutions for nonlinear systems with mean curvature-like operators, Nonlinear Anal. 65 (2006), 1462-1475. 2

[3] P. Benevieri, J. M. do Ó, E. Souto de Medeiros, Periodic solutions for nonlinear systems with mean curvature-like operators, Appl. Math. Lett. 20 (2006), 484-492. 2

[4] C. Bereanu and J. Mawhin, Nonhomogeneous boundary value problems for some nonlinear equations with singular $\varphi$-laplacian, J. Math. Anal. Appl. 352 (2009), 218-233. 2, 20

[5] C. Bereanu and J. Mawhin, Periodic solutions of nonlinear perturbations of $\varphi$-laplacians with possibly bounded $\varphi$, Nonlinear Anal. 68 (2008), 1668-1681. 2, 20

[6] C. Bereanu and J. Mawhin, Boundary value problems for some nonlinear systems with singular $\varphi$-laplacian, J. Fixed Point Theory Appl. 4 (2008), 57-75. 1, 2

[7] C. Bereanu and J. Mawhin, Existence and multiplicity results for some nonlinear problems with singular $\varphi$-laplacian, J. Differential Equations. 243 (2007), 536-557. 2, 20, 63

[8] C. Bereanu and J. Mawhin, Boundary-value problems with non-surjective $\varphi$-laplacian and onesided bounded nonlinearity, Advances Differential Equations. 11 (2006), 35-60. 2, 19, 20, 31, $36,39,73$

[9] C. Bereanu and J. Mawhin, Nonlinear Neumann boundary value problems with $\varphi$-laplacian operators, An. Stiint. Univ. Ovidius Constanta. 12 (2004), 73-92. 2

[10] V. Bouches and J. Mawhin, Boundary value problems for a class of first order quasilinear ordinary differential equations, Portugal. Math. (N.S). 71 (2014), 217-247. 2, 41

[11] L. E. J. Brouwer, Über Abbildung von Mannigfaltigkeiten, Match. Ann. 71 (1912), 97-115. 6

[12] J. Chandra, V. Lakshmikantham, and A. R. Mitchell, Existence of solutions of boundary value problems for nonlinear second-order systems in a Banach space, Nonlinear Anal. 2 (1978), 157-168. 9, 54, 59, 71

[13] A. da Costa, A construção do grau topológico e sua aplicação a um sistema diferencial não linear com condições de contorno, Dissertação de Mestrado em Matemática, USP, São Paulo. 2014. $6,7,8,11,13$

[14] G. Darbo, Punti uniti in trasformazioni a codominio non compatto, Rend. Sem. Mat. Univ. Padova. 24 (1955), 84-92. 14

[15] K. Deimling, Nonlinear Functional Analysis, Springer, Berlin, 1985. 5, 6, 8, 11, 12, 13, 14, 15, $16,30,34,36,46$

[16] A, Dold, Lectures on algebraic topoly, Springer, New York, 1980. 6 
[17] I. Fonseca and W. Gangbo, Degree theory in Analysis and applications, New York: Oxford Science Publications. 1995. 6, 11

[18] P. Girg, Neumann and periodic boundary-value problems for quasilinear ordinary differential equations with a nonlinearity in the derivative, Electronic J. Differential Equations. 63 (2000), 1-28. 2

[19] D. Guo, Y. Je Cho and J. Zhu, Partial ordering metods in nonlinear problems, Hauppauge, New York, 2004. 8, 9, 10, 14, 16, 17, 53

[20] C. Kuratowski, Monografie Matematyczne, vol. 20, Państwowe Wydawnictwo Naukowe, Warsaw, 1958. 14

[21] J. Leray and J. Schauder, Topologie et équations fonctionnelles, Ann. Sci. École Norm. Sup. 51 (1934), 45-78. 11

[22] E. Lima, Curso de Análise, Rio de Janeiro:vol. 2, Impa. 2009. 5

[23] N.G. Lloyd, Degree theory, Cambridge University Press, Cambridge, 1978. 11

[24] R. Manásevich and J. Mawhin, Periodic solutions for nonlinear systems with p-laplacian-like operators, Differential Equations. 145 (1997), 367-393. 2, 20

[25] J. Mawhin, Leray-Schauder degree:a half century of extensions and applications, Topological Methods Nonlinear Anal. 14 (1999), 195-228. 24

[26] J. Mawhin, Two point boundary value problems for nonlinear second order differential equations in Hilbert spaces, Tohoku Math. J. 32 (1980), 225-233. 63, 71

[27] J. Mawhin, Topological Degree Methods in Nonlinear Boundary Value Problems, CBMS series No. 40, American Math. Soc., Providence RI, 1979. 46

[28] H. Mönch, Boundary value problems for nonlinear ordinary differential equations of second order in Banach spaces, Nonlinear Analysis. 4 (1980), 985-999. 54, 71

[29] R.D. Nussbaum, Degree theory for local condensing maps, J. Math. Anal. Appl. 37 (1972), 741-766. 14

[30] R.D. Nussbaum, The fixed point index for local condensing maps, Ann. Mat. Pura Appl. 89 (1971), 217-258. 14

[31] R.D. Nussbaum, The fixed point index and asymptotic fixed point theorems for $k$-setcontractions, Bull. Amer. Math. Soc. 75 (1969), 490-495. 14

[32] W. Okrasiński and L. Plociniczak, A nonlinear mathematical model of the corneal shape, Nonlinear Anal. 13 (2012), 1498- 1505. 2

[33] D. P. D. Santos, Existence of solutions for some nonlinear problems with boundary value conditions, Abstract and Applied Analysis. doi:10.1155/2016/5283263. iii, v, 28, 35

[34] D. P. D. Santos, Existence of solutions to nonlinear problems with three-point boundary conditions, Electron. J. Differential Equations. 35 (2017), 1-10. iii, v

[35] D. P. D. Santos, Problems with mixed boundary conditions in Banach spaces, Chinese Journal of Mathematics, to appear. iii, v

[36] D. P. D. Santos, Problems with mean curvature-like operators and three-point boundary conditions, arXiv:1610.02461, 2016. iii, v 
[37] D. P. D. Santos, Existence results for nonlinear problems with classic homeomorphisms and nonlocal boundary conditions, submitted, 2017. iii, v

[38] A. Sard, The measure of the critical points of differentiable maps, Bull. Amer. Match. Soc. 48 (1942), 883-890. 5

[39] J. Schauder, Der Fixpunktsatz in Funktionenräumen, Studia Math. 2 (1930), 171-180. 13

[40] S. Szufla, Boundary value problems for nonlinear ordinary differential equations of second order in Banach spaces, Nonlinear Analysis. 8 (1984), 1481-1487. 54, 71

[41] G. Shilov, Elementary functional analysis, Cambridge, Mass.: MIT Press, 1974. 8, 10

[42] WX. Zhou and J. Peng, Existence of solution to a second-order boundary value problem via noncompactness measures, Discrete Dyn. Nat. Soc. Article ID 786404(2012). doi:10.1155/2012/786404. 63, 71 